\title{
Experimental Investigation of Emulsion AGET ATRP of MMA in a Stirred Tank Reactor
}

\author{
By \\ Kishor Regmi \\ M.Sc., Tribhuvan University, Kathmandu, Nepal, 1999 \\ B.Sc., Tribhuvan University, Kathmandu, Nepal, 1997
}

\author{
A Thesis \\ Presented to Ryerson University \\ in Partial fulfillment of the requirements for the Degree of \\ Master of Applied Science (MASc) \\ in the program of Chemical Engineering \\ Toronto, Ontario, Canada, 2016 \\ (C) Kishor Nath Upadhayay (Regmi), 2016.
}




\section{Author's Declaration}

I hereby declare that I am the sole author of this thesis. This is a true copy of the thesis, including any required final revisions, as accepted by my examiners.

I authorize Ryerson University to lend this thesis to other institutions or individuals for the purpose of scholarly research.

I further authorize Ryerson University to reproduce this thesis by photocopying or by other means, in total or in part, at the request of other institutions or individuals for the purpose of scholarly research.

I understand that my thesis may be made electronically available to the public.

Author's Signature

Kishor N. Up. Regmi 


\begin{abstract}
Experimental Investigation of Emulsion AGET ATRP of MMA in a stirred tank reactor

Kishor Nath Upadhayay Regmi

Master of Applied Science, 2016

Department of Chemical Engineering

Ryerson University

This study investigates the emulsion AGET ATRP of MMA in a 2-L reactor using the reactants: surfactant (Brij 98), catalyst complex $\left(\mathrm{CuBr}_{2} / \mathrm{dNbpy}\right)$, initiator $(\mathrm{EBiB})$ and reducing agent (ascorbic acid). Preliminary trials demonstrate that the two-step procedure preserves the ATRP living features much better than the single-step procedure. An experimental design and statistical analysis were performed to investigate the main effects and two-factor interaction effects of temperature, surfactant, catalyst complex, initiator and reducing agent on the monomer conversion, average molecular weights and polydispersity index of the polymer. The input-output model predictions agree with experimental data. The results revealed that the temperature was the most influential factor for all three-process responses with $71.34 \%$, $32.78 \%$ and $27.76 \%$ contribution. However, the initiator was the least influential factor for both conversion and PDI with $0.035 \%$ and $0.13 \%$ contribution, whereas the surfactant was the least influential factor for molecular weight with $0.068 \%$ contribution.
\end{abstract}




\section{ACKNOWLEDGMENTS}

I am indebted to my supervisor, Professor Ramdhane Dhib, for his invaluable guidance, support and insight. I consider myself fortunate to have had the chance to learn from such an excellent

mentor. I would also like to express my gratitude to my co-supervisor, Professor Mehrab Mehrvar, for his support and encouragement during the course of my research. I extend my thanks to my thesis committee members: Professor Anne E. Johnson and Professor Yaser Dahman for their time and consideration.

I also thank the engineering specialists Mr. Ali Hemmati, Mr. Daniel Boothe and Mr. Tondar Tajrobekar for their technical assistance and the administration staff for their help during my graduate studies in the Department of Chemical Engineering at Ryerson University.

My sincere gratitude goes out to my colleague and friend Mr. Ethan Massicotte for sharing his seemingly unending wealth of computer knowledge and making life in graduate school much more enjoyable. I wish to thank the former and current student colleagues at Ryerson University for their cherished friendship throughout these years.

Finally, and most importantly, I am extremely grateful to my beloved wife, Kanta, for her everlasting patience, love and support and to my dearest children Srishail and Saanvi for their goodness and patience throughout my years of study. Continuous backing, patience, understanding and encouragement of my family made this thesis possible. I would have found this achievement much more difficult without the support of my family. 


\section{DEDICATION}

This thesis is dedicated to my:

Beloved wife, Kanta

Father, Shiva N. UP. Regmi

Mother, Pabitra Regmi

Son, Srishail Regmi

And daughter, Saanvi Regmi. 


\section{TABLE OF CONTENTS}

AUTHOR'S DECLARATION.................................................................................................................................. i

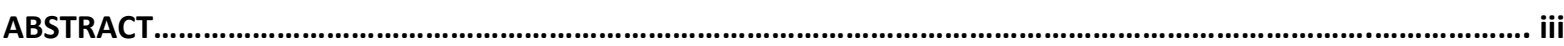

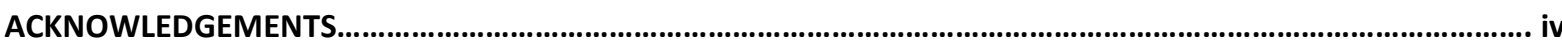

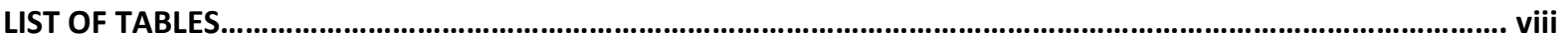

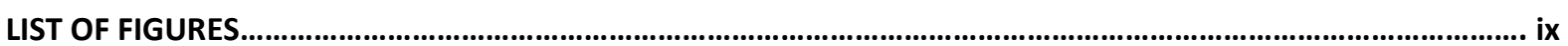

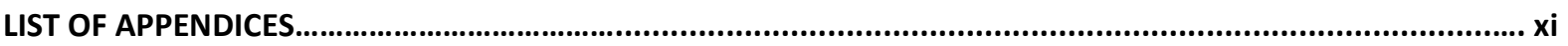

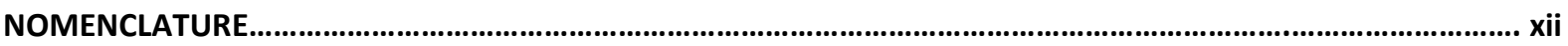

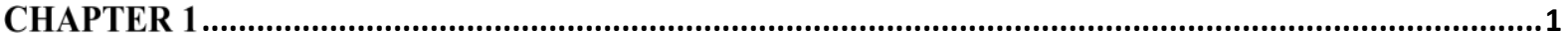

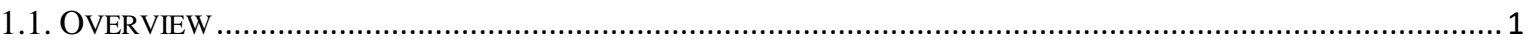

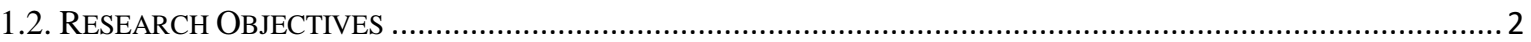

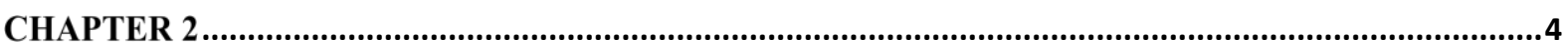

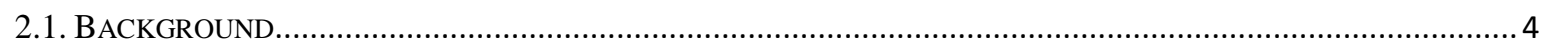

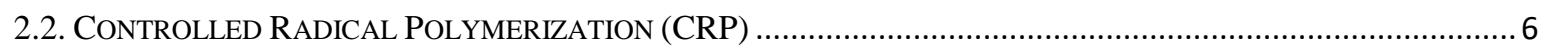

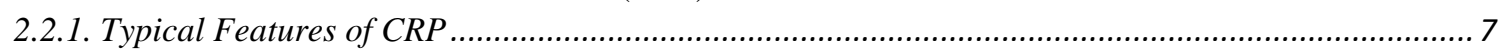

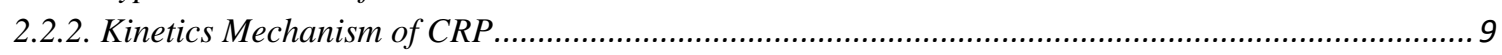

2.2.3. Approaches towards Controlled Radical Polymerization ............................................................. 10

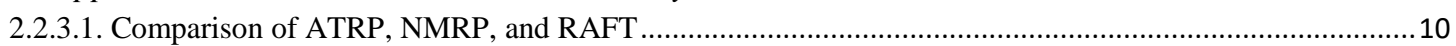

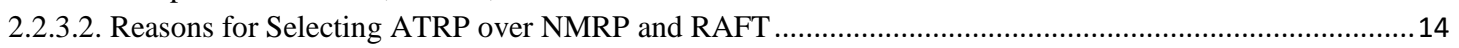

2.3. ATOM TRANSFER RADICAL POLYMERIZATION (ATRP) ............................................................ 15

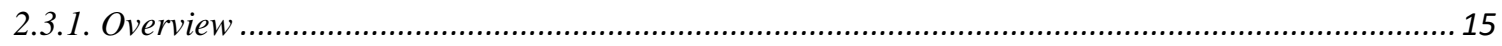

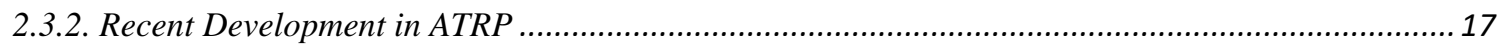

2.3.3. Recent Advances of ATRP in Aqueous Dispersed Media ........................................................... 19

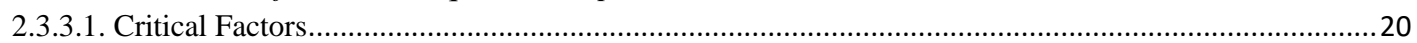

2.3.3.2. Successful Initiation Techniques for ATRP in Aqueous Dispersed Media .........................................21

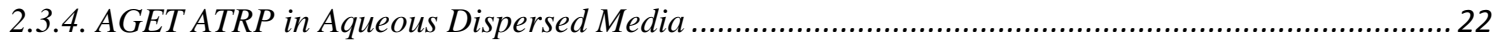

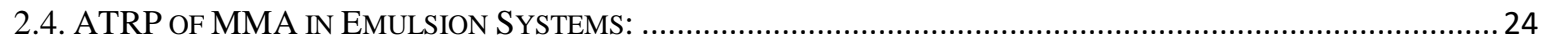

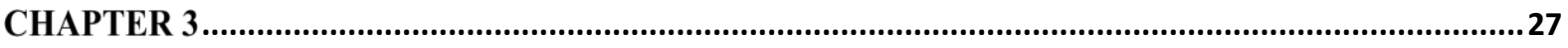

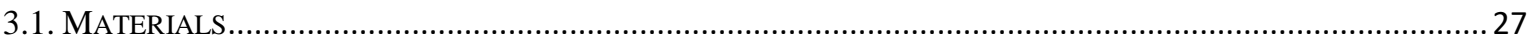

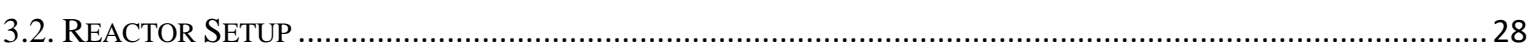

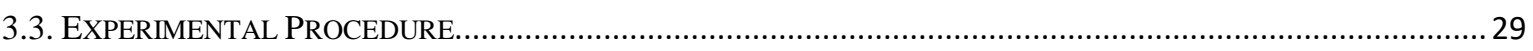

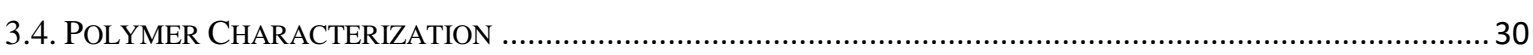

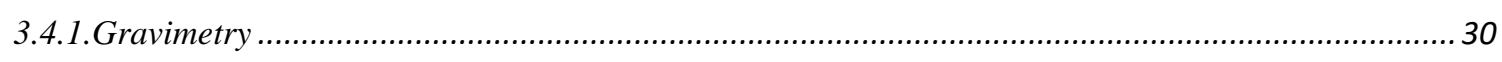

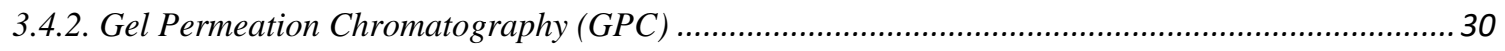

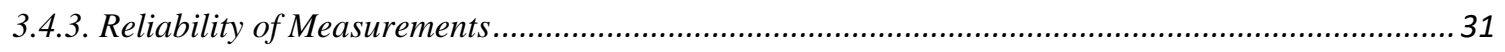

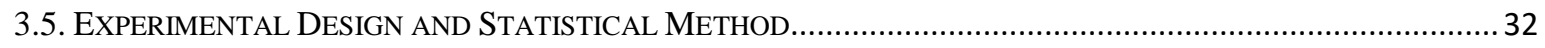

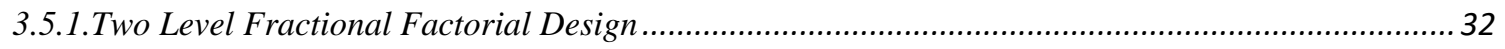

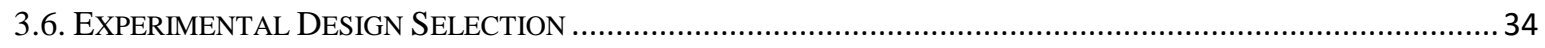

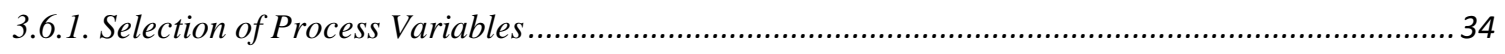

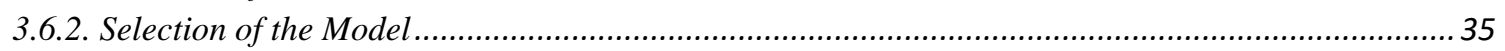

3.6.3. Statistical Method for Confirmation of the Model Accuracy ...................................................... 36 


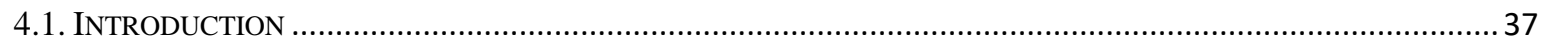

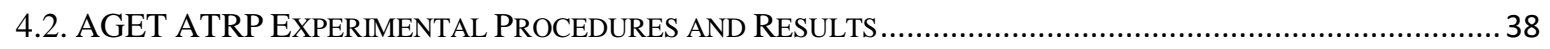

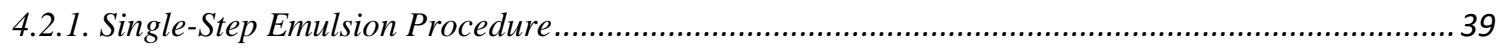

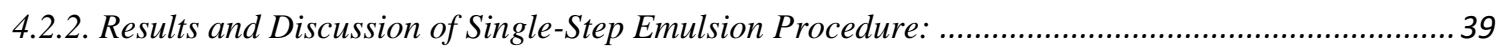

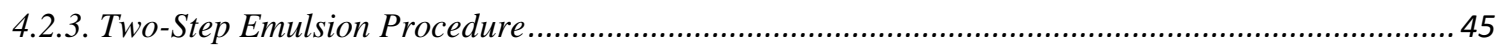

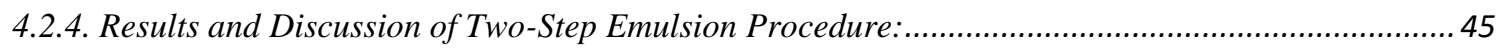

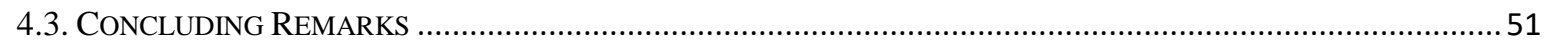

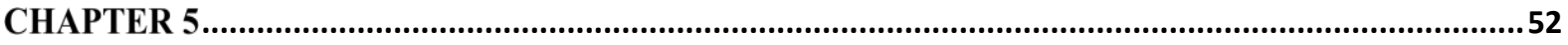

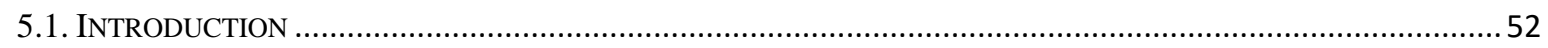

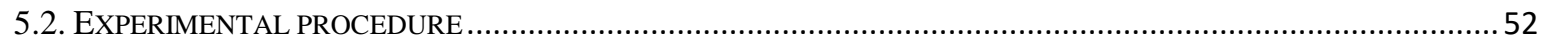

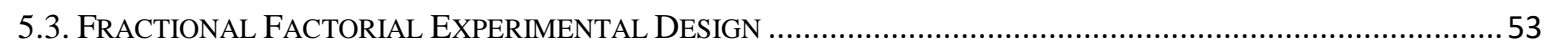

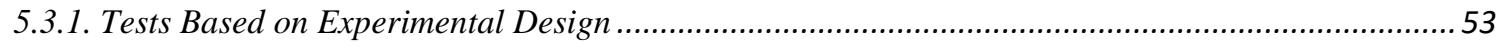

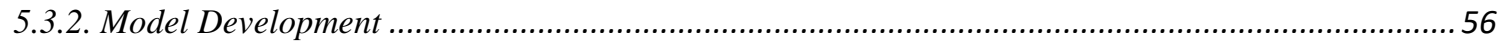

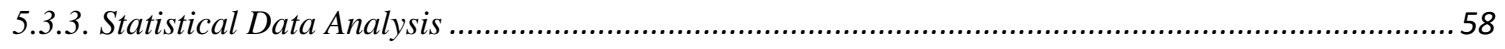

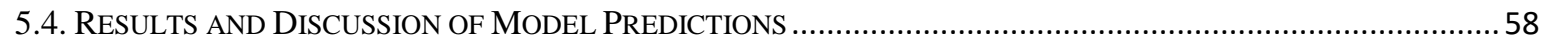

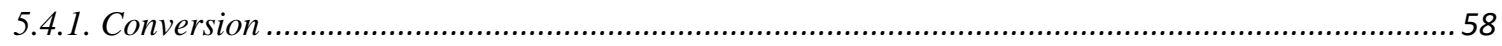

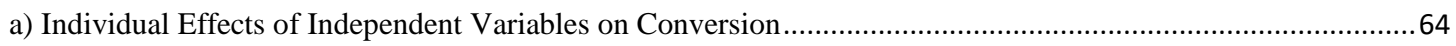

b) Effects of Interactions of Independent Variables on Conversion ....................................................................66

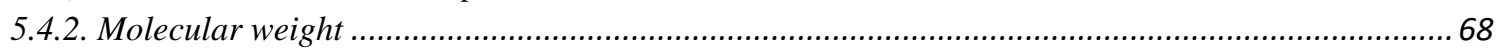

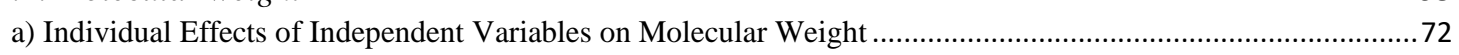

b) Effects of Interactions of Independent Variables on Molecular Weight ............................................................75

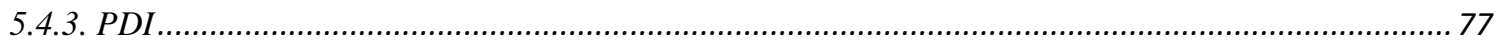

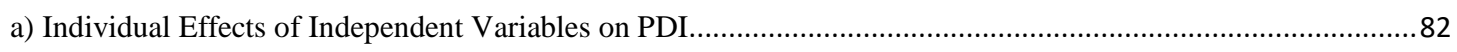

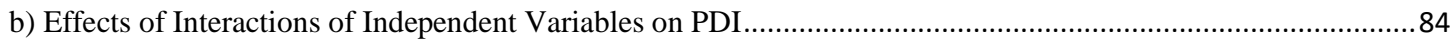

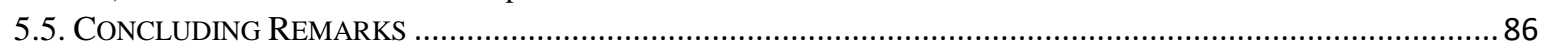

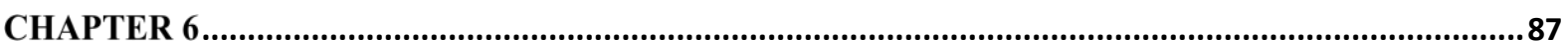

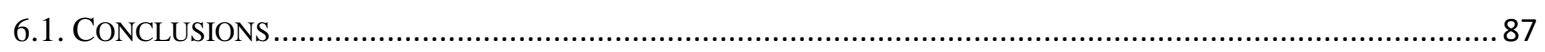

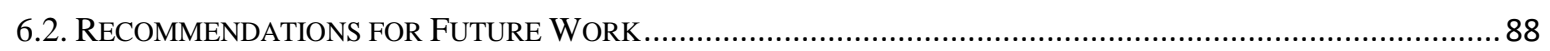

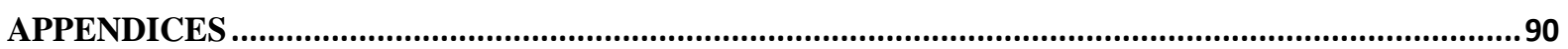

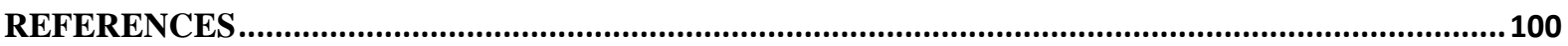




\section{LIST OF TABLES}

Table 2.1: Comparison of ATRP, NMRP and RAFT

Table 3.1: Reaction materials for emulsion AGET ATRP of MMA

Table 4.1: Recipe for the single-step emulsion AGET ATRP of MMA.

Table 4.2: Experimental conditions for the single-step emulsion AGET ATRP of MMA. .40

Table 4.3: Experimental results for single-step emulsion AGET ATRP of MMA

Table 4.4: Recipe for the two-step emulsion AGET ATRP of MMA 46

Table 4.5: Experimental conditions for the two-step emulsion AGET ATRP of MMA 46

Table 4.6: Experimental results for two-step emulsion AGET ATRP of MMA..... 47

Table 5.1: Experimental range and coded level of the independent variables employed in $\quad 2_{\mathrm{v}}{ }^{5-1}$ fractional factorial design.

Table 5.2: Five factor two-level fractional factorial experimental design with .54

Table 5.3: Experimental results of the measured responses

Table 5.4: Regression coefficients and probability values of statistical analysis for the prediction of the response variables.

Table 5.5: ANOVA summary of the model for conversion, molecular weight and PDI. .58

Table 5.6: Effects list showing \% contribution of main factors and two factor interaction effects on conversion.

Table 5.7: Effects list showing \% contribution of main factors and two factor interaction effects on molecular weight.

Table 5.8: Effects list showing \% contribution of main factors and two factor interaction effects on PDI.78 


\section{LIST OF FIGURES}

Figure 2.1: Contributing areas for the development of CRP.

Figure 2.2: First order kinetic plot for controlled radical polymerization. ................................................. 8

Figure 2.3: Average molecular weight for different types of reactions. ................................................. 8

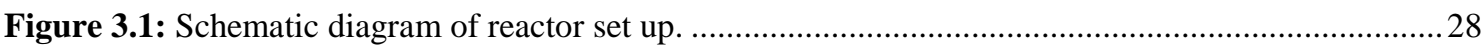

Figure 4.1: MMA conversion versus time in single-step emulsion polymerizations.................................43

Figure 4.2: Variations of $\ln \left([\mathrm{M}]_{0} /[\mathrm{M}]\right)$ with reaction time in experiments $\mathrm{S} 6$ and $\mathrm{S} 7$.

Figure 4.3: Variations of experimental number-average molar mass and polydispersity index (PDI) versus MMA conversions in expt. S7.

Figure 4.4: A, B, C: GPC traces of PMMA from expt. S7 for $35.5 \%, 39.3 \%$ and $64.8 \%$ conversions, respectively

Figure 4.5: MMA conversion versus time in two-step emulsion polymerizations.

Figure 4.6: Variations of $\ln \left([\mathrm{M}]_{\mathrm{o}} /[\mathrm{M}]\right)$ with reaction time in two-step emulsion polymerizations. 48

Figure 4.7: Dependence of experimental number-average molar mass $\left(\mathrm{M}_{\mathrm{n}}, \mathrm{g} \mathrm{mol}^{-1}\right)$ and polydispersity index (PDI) on MMA conversions for experiment T2

Figure 4.8: Dependence of experimental number-average molar mass $\left(\mathrm{M}_{\mathrm{n}}, \mathrm{g} \mathrm{mol}^{-1}\right)$ and polydispersity index (PDI) on MMA conversions for experiment T3

Figure 4.9: A, B, C: GPC traces of PMMA from experiment $\mathrm{T} 3$ for $12.8 \%, 24.7 \%$ and $34.8 \%$

conversions respectively.

Figure 4.10: Aggregation/Coagulation in: A) Single-Step emulsion and B) two-step emulsion. 50

Figure 5.1: Half normal probability plot for the selection of main factors and two factor 59

Figure 5.2: Pareto chart with the list of effects according to their influence level on conversion. 60

Figure 5.3: Diagnostic plots for conversion: (A) normal probability plot of residuals, (B) residuals vs predicted (C) residuals vs run and (D) predicted vs actual. 
Figure 5.4: Effects of process inputs on the conversion

Figure 5.5: Interaction of process inputs and their effects on the conversion

Figure 5.6: Half normal probability plot for the selection of main factors and two factor interaction effects influencing polymer molecular weight

Figure 5.7: Pareto chart with the list of effects according to their influence level on the molecular weight.69

Figure 5.8: Diagnostic plots for molecular weight: (A) normal probability plot of residuals, (B) residuals vs predicted (C) residuals vs run, and (D) predicted vs actual

Figure 5.9: Individual effects of process inputs on the average molecular weight of the polymer

(PMMA)

Figure 5.10: Interaction of process inputs and their effect on the average molecular weight of the polymer

(PMMA) 76

Figure 5.11: Half normal probability plot for the selection of main factors and two factor interaction effects influencing PDI.

Figure 5.12: Pareto chart with the list of effects according to their influence level on PDI.

Figure 5.13: Diagnostic plots for PDI: (A) normal probability plot of residuals, (B) residuals vs predicted, (C) residuals vs run and (D) predicted vs actual

Figure 5.14: Individual effects of process inputs on the PDI.

Figure 5.15: Interaction of process inputs and their effect on the PDI 


\section{LIST OF APPENDICES}

Appendix A: Recent Developments in ATRP..................................... 900

Appendix B: Polymerization in aqueous dispersed media......................... 92

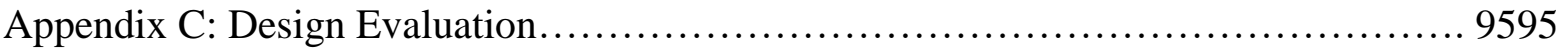

Appendix D: Statistical Analysis............................................ 97 


\section{NOMENCLATURE}

AA Ascorbic acid

AGET Activators generated by electron transfer

ANOVA Analysis of variance

ARGET Activators regenerated by electron transfer

ATRP Atom transfer radical polymerization

$b_{i} \quad$ Regression coefficients for the linear term

$b_{i j} \quad$ Regression coefficients for the interaction term

$b_{0} \quad$ Regression coefficients at the intercept

BPMODA Bis (2-pyridylmethyl) octadecylamine

Bpy 2,2'-Bipyridine

Brij 98 Polyoxyethylene (20) oleyl ether

CMC Critical micellar concentration

Conv Conversion, $\%$

CRP Controlled (living) radical polymerization

CTAB Cetyltrimethylammonium bromide

DMCBCy dimethylated 1,8-ethylene cross-bridged 1,4,8,11-tetraazacyclotetradecane

$\mathrm{dn} / \mathrm{dc} \quad$ Specific refractive index increment

dNbpy 4,4'-Dinonylbipyridine

DOE Design of experiments

DP Degree of polymerization

EBiB Ethyl-2-bromoisobutyrate

FFD Fractional factorial design

FRP Conventional free radical polymerization

GPC Gel permeation chromatography

[I] Molar concentration of initiator, mol/L

$[\mathrm{I}]_{\mathrm{o}} \quad$ Initial initiator concentration, $\mathrm{mol} / \mathrm{L}$

I $\quad$ Initiator radical or primary radical

ICAR Initiators for continuous activator regeneration

I-X ATRP initiator 
$\mathrm{k}_{\text {act }} \quad$ Activation rate constant, $\mathrm{L} / \mathrm{mol} \mathrm{s}$

$\mathrm{k}_{\text {deact }} \quad$ Deactivation rate constant, $\mathrm{L} / \mathrm{mol} \mathrm{s}$

$\mathrm{k}_{\mathrm{i}} \quad$ Rate constant of initiation, $\mathrm{L} / \mathrm{mol} \mathrm{s}$

$\mathrm{k}_{\mathrm{p}} \quad$ Propagation (polymerization) rate constant, $\mathrm{L} / \mathrm{mol} \mathrm{s}$

$\mathrm{k}_{\mathrm{p}} \quad$ Rate constant of propagation, $\mathrm{L} / \mathrm{mol} \mathrm{s}$

$\mathrm{k}_{\mathrm{t}} \quad$ Termination rate constant, $\mathrm{L} / \mathrm{mol} \mathrm{s}$

$\mathrm{k}_{\mathrm{tc}} \quad$ Rate constant of termination by combination, $\mathrm{L} / \mathrm{mol} \mathrm{s}$

$\mathrm{k}_{\mathrm{td}} \quad$ Rate constant of termination by disproportionation, $\mathrm{L} / \mathrm{mol} \mathrm{s}$

$\mathrm{k}_{\text {tr }} \quad$ Rate constant of chain transfer, L/mol s

Li Ligand

LALLS Low angle laser light scattering

M Monomer

[M] Molar concentration of monomer, mol/L

$[\mathrm{M}]_{\mathrm{o}} \quad$ Initial monomer concentration, $\mathrm{mol} / \mathrm{L}$

MA Methyl acrylate

$\mathrm{Me}_{6}$ TREN Tris [2- (N, N-dimethylamino) ethyl] amine

MEHQ Mono methyl ether hydroquinone

MMA Methyl methacrylate

$\mathrm{M}_{\mathrm{n}} \quad$ Number-average molecular weight of the polymer, $\mathrm{g} / \mathrm{mol}$

$\mathrm{Mt}^{\mathrm{q}} / \mathrm{Li} \quad$ Transition metal complex in lower oxidation state

$\mathrm{Mt}^{\mathrm{q}+1} / \mathrm{Li} \quad$ Transition metal complex in higher oxidation state

$\mathrm{M}_{\mathrm{w}} \quad$ Weight-average molecular weight of the polymer, $\mathrm{g} / \mathrm{mol}$

MW Molecular weight

MWD Molecular weight distribution

NMRP Nitroxide mediated radical polymerization

OS Oxidized species

PDI Polydispersity index $\left(\mathrm{M}_{\mathrm{w}} / \mathrm{M}_{\mathrm{n}}\right)$

$\mathrm{P}_{\mathrm{m}} \quad$ Dead polymers of length $\mathrm{m}$

PMDETA N, N, N', N' N',- Pentamethyldiethylenetriamine

PMMA Polymethyl methacrylate

$\mathrm{P}_{\mathrm{n}} \quad$ Dead polymers of length $\mathrm{n}$

$\mathrm{P}_{\mathrm{n}+\mathrm{m}} \quad$ Dead polymers of length $\mathrm{n}+\mathrm{m}$ 
$\left[\mathrm{P}_{\mathrm{n}}{ }^{\cdot}\right]$ Concentration of active propagating species, $\mathrm{g} / \mathrm{mol}$

$p$-value Probability value

PSD Particle size distribution

$\mathrm{R}_{1} \cdot \quad$ Propagating radical of length 1

$\mathrm{R}^{2} \quad$ Coefficient of determination

$\mathrm{R}^{2}$ adj $\quad$ Adjusted regression coefficient

$\mathrm{R}_{\text {pred }}^{2} \quad$ Predicted regression coefficient

RA Reducing agent

RAFT Reversible addition-fragmentation chain transfer

RALLS Right-angle laser light scattering detectors

RI Refractive index

$\mathrm{R}_{\mathrm{n}} \mathrm{B}^{-} \quad$ Propagating radical of size $\mathrm{n}$

$\mathrm{R}_{\mathrm{n}}-\mathrm{X} \quad$ Halide capped polymer

S Solvent

$\mathrm{Sn}(\mathrm{EH})_{2} \quad$ Tin (II) 2-ethylhexanoate

SR\&NI Simultaneous reverse and normal initiation

St Styrene

T Time, s, min, h

THF Tetrahydrofuran

tNtpy 4',4"-Tris(5-nonyl)-2,2':6',2"-terpyridine

TPMA Tris(2-pyridylmethyl) amine

X Halogen atom

$x_{1}$ or (A) Temperature

$x_{2}$ or (B) Catalyst complex $\left(\mathrm{CuBr}_{2} / \mathrm{dNbpy}\right)$

$x_{3}$ or (C) Surfactant (Brij 98)

$x_{4}$ or (D) Initiator (EBiB)

$x_{5}$ or (E) Reducing agent (ascorbic acid)

$y_{1} \quad$ Conversion, $\%$

$y_{2} \quad$ Polymer molecular weight, $\mathrm{g} / \mathrm{mol}$

$y_{3} \quad$ Polydispersity index (PDI)

$\lambda_{0} \quad$ Total concentration of all live radicals 


\section{CHAPTER 1}

\section{INTRODUCTION}

\subsection{Overview}

In the field of polymer chemistry, Controlled Radical Polymerization (CRP) is one of the most rapidly developing areas. The combined advantages of Free Radical Polymerization (FRP) and living ionic polymerization have allowed the economic production of new polymers and are probably the main reason for growing academic and industrial interests in CRP.

Among the three major routes of CRP, Atom Transfer Radical Polymerization (ATRP) is one of the most popular polymerizations. In fact, polymers synthesized by ATRP processes, in both academic and industrial settings, can be used as lubricants, surfactants, adhesives, inks, gels, dispersants, additives, thermoplastic elastomers, as well as for biomedical applications such as drug delivery and artificial bones (Braunecker and Matyjaszewski, 2007; Destarac, 2010; Matyjaszewski, 2014; Oh, 2008).

ATRP has been successfully carried out in homogeneous conditions and also in various dispersed media. In particular, ATRP in aqueous dispersed media has several advantages: use of environmentally friendly and economic dispersant, very efficient heat transfer, less viscous reaction medium allowing high conversion and sometimes direct application of the latex (Cunningham, 2008; Li, 2012; Min, 2008; Oh, 2008).

Although the literature on ATRP in aqueous dispersed media (emulsion ATRP) is not extensive, it is growing rapidly. However, the chemistry and other kinetic/mechanistic aspects of ATRP in emulsion are still not well understood.

The objective of this thesis is to carry out experimental investigations on the single-step and two-step emulsion polymerizations of methyl methacrylate (MMA) under different conditions. First, a preliminary experimental investigation of the process has been performed using the single-step and two-step emulsion polymerization of methyl methacrylate in laboratory scale batch reactor. Secondly, the procedure consists of assessing the main effects and two-factor interaction effects of five factors: temperature, catalyst complex $\left(\mathrm{CuBr}_{2} / \mathrm{dNbpy}\right)$, initiator 
(EBiB), reducing agent (ascorbic acid) and surfactant (Brij 98) on the conversion, PDI and polymer molecular weight averages in the two-step emulsion polymerization

Chapter 2 is organized into two sections. The first one is devoted to debate the research topic background and literature review of CRP, ATRP, polymerization in aqueous dispersed media, Activators Generated by Electron Transfer (AGET) ATRP polymerization in emulsions system and also ATRP of MMA in emulsion systems. In the second part, the research objectives are outlined.

Chapter 3 encompasses the laboratory-scale experimental set up of the 2-L stirred tank reactor system, experimental procedures, polymer characterization methods and selection of the design of experiments. Considering the fact that this polymerization procedure is relatively new, an experimental design was set up to develop a sound analysis of the MMA ATRP process. A quantitative analysis is done in this study.

Chapter 4 explores in details the AGET ATRP of MMA in a single-step and two-step emulsion polymerizations. Both polymerizations are carried out in the 2-L stirred tank reactor. Preliminary experimental tests were performed and served as an experimental evidence to opt out for the two-step emulsion polymerization.

Chapter 5 discusses the experimental procedure to perform the two-step emulsion AGET ATRP polymerization of MMA. Resolution five fractional factorial $\left(2{ }_{\mathrm{v}}{ }^{5-1}\right)$ experimental design was employed to conduct the necessary experimental tests that allow the development of an input-output model. Also, a statistical analysis is discussed.

Finally, the main concluding remarks and recommendations for future investigation on the topic are summarized in Chapter 6.

\subsection{Research Objectives}

ATRP is a powerful tool to produce polymers with controlled molecular weight and narrow polydispersity. MMA is well established and widely studied for its versatile applications. Emulsion polymerization is a high caliber choice in industry for its environmental and economic benefits as compared to bulk and solution polymerizations. 
This study focuses on the investigation of AGET ATRP polymerization of MMA in a 2-liter reactor system. The reaction has been performed separately in a single step and two-step emulsion procedures. The reaction recipient consists of distilled water as a continuous phase, MMA as monomer, $\mathrm{CuBr}_{2} / \mathrm{dNbpy}$ as catalyst complex, Brij 98 as surfactant, ascorbic acid (AA) as reducing agent, and $\mathrm{EBiB}$ as initiator.

The major objectives of the thesis are:

1. To perform a preliminary experimental investigation of single-step and two-step emulsion AGET ATRP polymerization of MMA in the stirred tank batch reactor in order to determine the feasibility and limitations of the reaction.

2. To use the outcome of the preliminary experiments as a guide to select which of the two methods, single-step or two-step, is more appropriate for further investigation.

3. To collect new data of MMA polymerization using the procedure selected in 2 (above), based on an experimental design.

4. To carry out statistical analysis of the results (conversion, PDI, and molecular weight) for the estimation of the main effects and two-factor interactions of the independent variables in the process outputs considering the independent variables as: temperature, catalyst complex $\left(\mathrm{CuBr}_{2} / \mathrm{dNbpy}\right)$, initiator $(\mathrm{EBiB})$, reducing agent (ascorbic acid) and surfactant (Brij 98) and the process dependent variables as: monomer conversion and polymer $\mathrm{M}_{\mathrm{n}}$ and PDI. 


\section{CHAPTER 2}

\section{LITERATURE REVIEW}

\subsection{Background}

Conventional free radical polymerization (FRP) is the most important and widely employed technique for the production of most engineering and commodity polymers. However, some inherent features of this polymerization do not allow the preparation of polymers of well controlled molecular weight (MW), molecular weight distribution (MWD), chain architecture and chain end functionality (Braunecker and Matyjaszewski, 2007; Matyjaszewski et al., 1997).

To overcome these shortcomings of FRP, ionic chain polymerization came into light (Szwarc, 1956). However, even though ionic chain polymerization has contributed a lot for the production of polymers with desired characteristics, it turned out to be inferior to radical polymerization in terms of range of monomers it can polymerize, the reaction conditions (range of temperature and solvent) it demands and rigorous purification of reagents it needs (Braunecker and Matyjaszewski, 2007; Eslami and Zhu, 2005; Min, 2008; Monteiro and Cunningham, 2012).

Consequently, it took few decades for researchers to pave the way towards the development of what is today known as the controlled radical polymerization (CRP). Georges (1993) was one of the pioneers, who started a new polymerization technique, called controlled radical polymerization (CRP) (Braunecker and Matyjaszewski, 2007; Greszta et al., 1994; Matyjaszewski, 2014; Matyjaszewski et al., 1997).

As shown in Figure 2.1, Controlled radical polymerization by encompassing some of the advantages of both FRP and living ionic polymerization has allowed the economic production of polymers with relatively good control of polydispersity, molecular weights and end group functionality (Braunecker and Matyjaszewski, 2007; Mastan et al., 2014; Matyjaszewski, 1997; Nabifar, 2007). Polymers prepared by CRP have potential applications in biomedicines and nanotechnology (Oh, 2008). 
Even though the kinetics of free radical intermediates in both FRP and CRP are fundamentally similar, it is important to shed light on the differences between them.

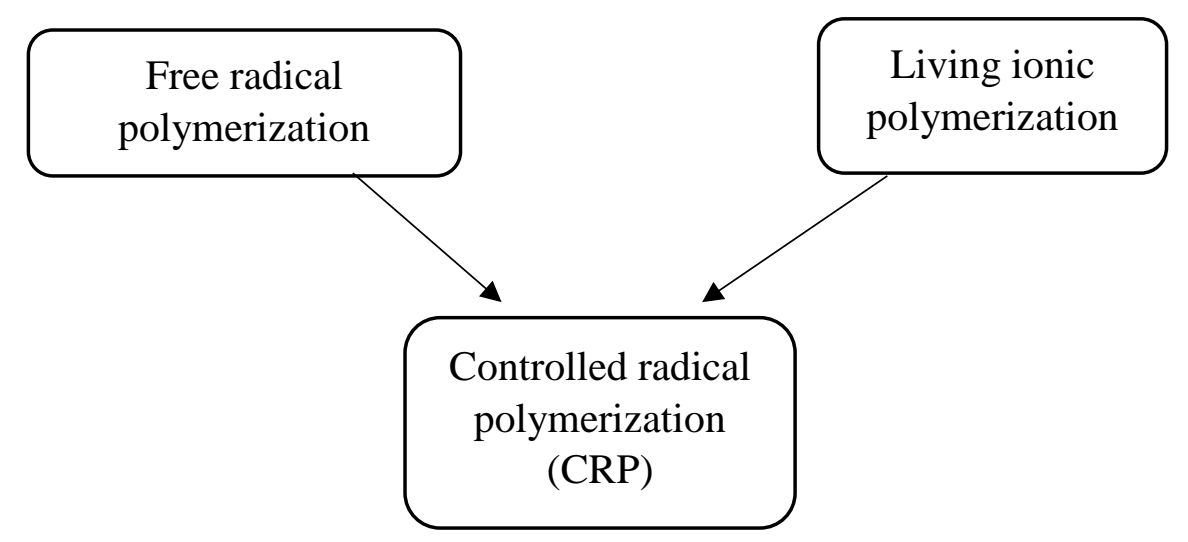

Figure 2.1: Contributing areas for the development of CRP.

Major reactions in FRP are discussed in the previous studies (Bebe, 2008; Bergenudd, 2011; Louie et al., 1985; Odian, 2004) and summarized below:

Chain initiation: Primary free radicals are usually produced by the thermal decomposition of an initiator (peroxides, persulphates, diazo compounds) or catalyst (Ziegler-Natta, metallocene). The primary radicals react with monomers to initiate growing polymer chains (chain legth unity). During the initiation phase, termination between primary radicals is a side reaction which can affect initiator efficiency.

Chain propagation: It is the main chain growth reaction, its rate is very high and it is assumed to be independent of the chain length.

Chain termination: occurs when two live polymer radicals react together, it can occur by disproportionation or combination. 


\subsection{Controlled Radical Polymerization (CRP)}

Controlled radical polymerization has expanded into different routes for the synthesis of a wide spectrum of polymers with well-defined molecular weights, low polydispersity index (PDI) and various polymer architecture under mild conditions with nominal purification of reagents. Currently, the most common and highly studied CRP methods are:

- Atom transfer radical polymerization (ATRP);

- Nitroxide-mediated radical polymerization (NMRP);

- Reversible addition-fragmentation chain transfer polymerization (RAFT).

A common feature of all CRP techniques is the existence of dynamic equilibrium reaction between active radicals and dormant species (Goto and Fukuda, 2004; Greszta et al., 1994). This equilibrium reaction (Equation 2.2, for ATRP) provocates a low concentration of radicals which significantly reduces termination, in the meantime it allows slow but simultaneous growth of all chains (Matyjaszewski and Xia, 2001).

To achieve good control of the reaction, the following conditions must be fulfilled.

1. Fast and instantaneous initiation of all chains (very high efficiency of initiator),

2. Negligible contribution of chain breaking reactions like chain transfer and termination, and

3. Fast exchange between active and dormant species.

Decomposition of the initiator should be almost instantaneous so that the propagating radicals have equal life time which obviously results in polymer chains of similar chain length, i.e., a PDI close to unity. This can be compared with the plantation of seeds; if all seeds were planted at the same time then it is likely that all of them grow to similar height at a given time provided that the rate of growth is constant. Bimolecular termination and chain transfer reactions are inherently important in FRP. But, they are suppressed in CRP (Shipp, 2005). There must be a fast exchange between active and dormant species and the equilibrium should favor dormant species (Matyjaszewski, 2002). Only a few propagation steps may be allowed for the active chains. When the dynamic equilibrium reaction is faster than the propagation reaction, then chain growth can increment, thus reducing chain transfer and narrowing the PDI. This can be compared to a constant rate of growth of planted seeds in the example above. 


\subsubsection{Typical Features of CRP}

Reversible deactivation reactions are at the core of CRP. The dynamic equilibrium reaction favouring dormant species makes termination negligible and increases radical life greatly. This in fact allows sufficient interplay during polymerization to control MW, MWD, PDI, and chain end functionality. Because of radical intermediates, CRP has the advantage of the relative comfort of FRP in terms of condition (purity and temperature). Likewise, because of the controlled trap of radicals, it has the advantages of living ionic polymerization in term of preserved chain end functionality, MW, MWD, and PDI. Almost instantaneous initiation and fast exchange among active and dormant species are always necessary for a good control over MW, PDI, and chain architecture in all CRP systems. Ideally each growing species should react only with few monomer units before its deactivation (Matyjaszewski and Xia, 2001; Nabifar, 2007).

According to the works of eminent researchers in the field, ideal CRP has the following features:

\section{First order kinetic behavior:}

A linear kinetic plot a shown (Figure 2.2$)$ in semi-logarithmic coordinates $\left(\ln \left([\mathrm{M}]_{\mathrm{o}} /[\mathrm{M}]\right)\right.$ versus time) is the typical feature of CRP.

Rate of polymerization, $\mathrm{R}_{\mathrm{p}}=\frac{-d[M]}{d t}=K_{p}[M] \lambda_{0}, \quad$ Where $\lambda_{0}=\sum_{n=1}^{\infty} R_{n}^{\bullet}$

$$
\ln \frac{[M]_{o}}{[M]}=k_{p} \lambda_{0} t
$$

If $\lambda_{0}$ is constant, then the equation above results in a linear dependence of $\ln \left([\mathrm{M}]_{\mathrm{o}} /[\mathrm{M}]\right)$ with time.

Where $[\mathrm{M}]_{\mathrm{o}}$ : Initial monomer concentration, $\mathrm{g} / \mathrm{mol}$

$[\mathrm{M}]:$ Monomer concentration at time $\mathrm{t}, \mathrm{g} / \mathrm{mol}$

$R_{n}^{\bullet}$ : Active propagating species,

$\lambda_{0}$ : Total concentration of all active propagating radicals, $\mathrm{g} / \mathrm{mol}$

$K_{p}$ : Propagation constant, $\mathrm{L} / \mathrm{mol} \mathrm{s}$

$t$ : Reaction time, $\mathrm{s}$ 
A linear trend is obtained when the concentration of the active propagating species is constant. Any curvature indicates disturbance in the concentration of active species by slow initiation, termination or other side reactions.

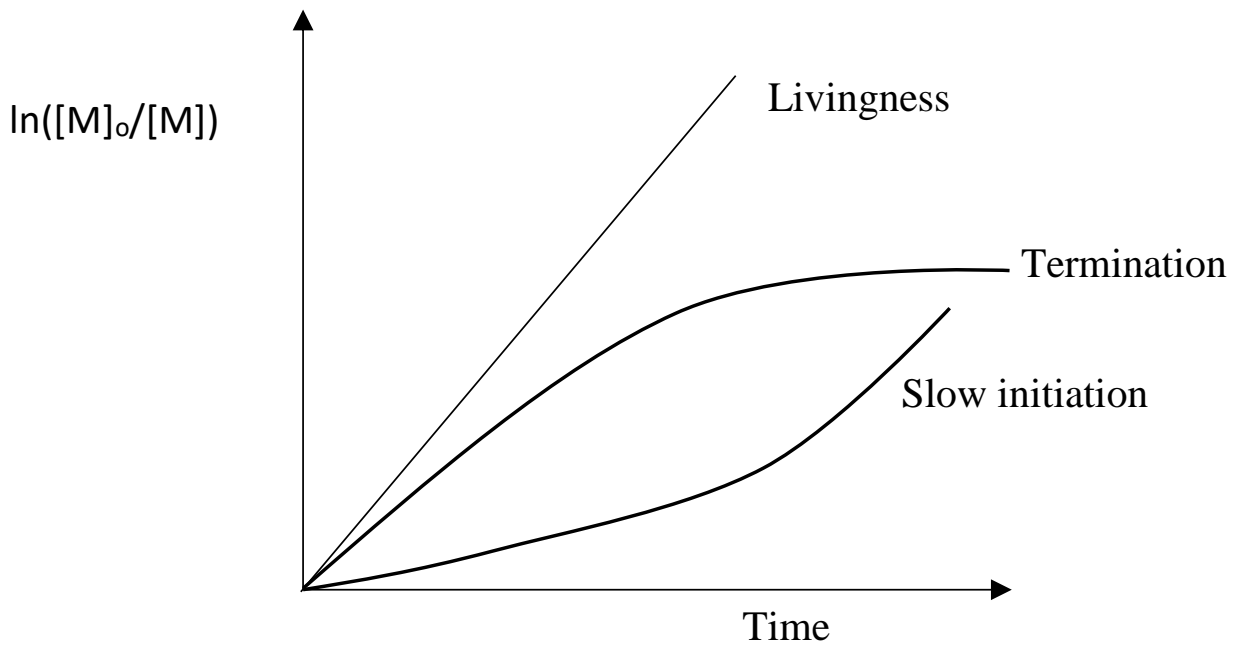

Figure 2.2: First order kinetic plot for controlled radical polymerization.

\section{Linear increase of average molecular weight with conversion:}

If the initiation is sufficiently fast and there is an absence of chain transfer reactions that increases the total number of chains, then the number average molecular weight $\left(\mathrm{M}_{n}\right)$ is a linear function of monomer conversion. This feature is significantly different in FRP as shown in figure 2.3 .

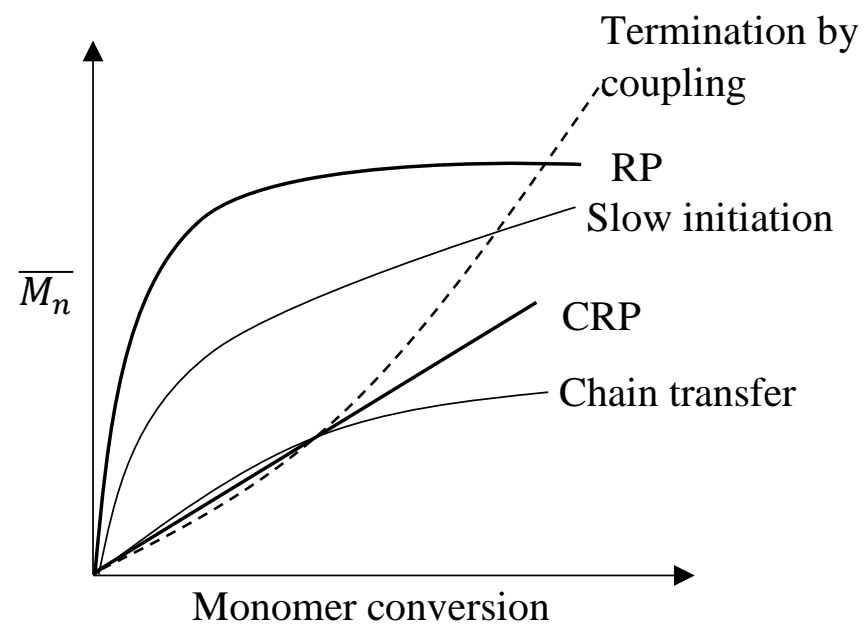

Figure 2.3: Average molecular weight for different types of reactions. 


\section{Designed, usually narrow, molecular weight distribution:}

Distribution of polymer chains of an ideal CRP system is close to Poisson distribution with PDI nearly unity. However, for real CRP, the PDI is usually expected to be less than 1.5 corresponding to the lowest limit for a free radical polymerization.

For the low PDI, the absence of the termination and the chain transfer are not the only requirements, it may also require the following (Coleman and Fox, 1963; Matyjaszewski, 1996):

a. Near instantaneous initiation of all chains allowing simultaneous growth of all the polymer chains,

b. Higher rate of exchange between active and dormant species than the rate of propagation, so that uniform chain growth is ensured,

c. Chain transfer and termination must be negligible,

d. The rate of depropagation should be negligible compared to that of propagation.

\section{Long-lived polymer chains with preserved end functionalities:}

Because of negligible chain transfer and termination, most of the chains may retain their active centers even after full consumption of the monomer. This allows the chain extension and preparation of block copolymers by sequential addition of monomer. The beauty of CRP lies in its ability to control the bulk properties of final material through control of composition, functionality and topology which is largely due to the preserved chain end functionality (Shipp, 2005).

\subsubsection{Kinetics Mechanism of CRP}

The kinetics mechanism of CRP is very much comparable to that of conventional radical polymerization. It, however, differs from conventional radical polymerization in that a chemical equilibrium is established between dormant and active species (radicals) by reversible trap of active species (radicals). All other steps (initiation, propagation, termination and chain transfer) are similar (Bergenudd, 2011; Chan et al., 2013; Coessens and Matyjaszewski, 2010; Louie et al., 1985; Matyjaszewski, 2012; Matyjaszewski and Xia, 2001; Pintauer and Matyjaszewski, 2008). 
Depending on the CRP procedure, the initiation can be done differently depending on the use of initiator: conventional radical initiator, halogen containing organic compounds, nitroxide capped species and so on.

The reduced termination and the chain transfer allows control over PDI, MWD and MW. For instance, the control in chain architecture and the chain end functionality are possible because of stable dormant species (Louie et al., 1985).

In regular radical polymerization (FRP), all four steps (initiation, propagation, termination and chain transfer) are assumed occurring concurrently and very fast. Thus, for continuous polymerization a suitable initiator that decomposes into radicals throughout the reaction must be chosen.

The slow initiation and fast propagation (short life time of radical $\sim 1 \mathrm{sec}$ ) can produce polymers of different chain length before termination resulting into broader polydispersity (higher than 1.5). Since every radical undergoes termination in a very short time, it is hard to incorporate end group functionality in polymer chains, thus not allowing controlled architecture (BarnerKowollik et al., 2002).

However, CRP has some distinct features allowing it to have control over MW, MWD and chain end functionality. Since the majority of chains are in the dormant state, it is very convenient to control the architecture of the polymer by modification in chain end functionality.

Unlike FRP, CRP has very fast initiation and prolonged radical lifetime (increased from seconds to hours or even days through the equilibrium between active and dormant species), which allows good control on MW and MWD. The reader can refer to Section 2.3.1 for kinetics mechanism of ATRP (one of the CRP methods).

\subsubsection{Approaches towards Controlled Radical Polymerization}

\subsubsection{Comparison of ATRP, NMRP, and RAFT}

ATRP and NMRP Both follow persistent radical effect (PRE). PRE is a specific chemical kinetics feature where propagating radicals are reversibly trapped in a deactivation process by a persistent radical species. Persistent radicals cannot terminate with each other, but they can cross-couple with growing species. The termination is not completely eliminated for radicals 
and every termination causes irreversible accumulation of persistent radicals; finally a condition will come where steady state of growing radicals is established through crosscoupling with persistent radicals. In systems obeying PRE, fast and near instantaneous initiation of all chains is necessay for low MWD or PDI (Ayres, 2011; Barner-Kowollik et al., 2002; Braunecker and Matyjaszewski, 2007; Coessens and Matyjaszewski, 2010; Cunningham, 2008, 2003; Goto and Fukuda, 2004; Guégain et al., 2015; Guillaneuf et al., 2006; Matyjaszewski, 2012; Murari et al., 2011; Pintauer and Matyjaszewski, 2008).

However, RAFT obeys degenerative transfer instead of PRE. Steady state of growing radicals in RAFT is maintained by initiation/termination which is different than systems with PRE. Nevertheless RAFT follows typical FRP kinetics with slow initiation and fast termination (Drache et al., 2005; Matyjaszewski and Xia, 2001; Ulitin et al., 2012; Vana et al., 2002; Zhang and Ray, 2001).

Kinetics and chain control in ATRP depends on both the persistent radical and the activator (lower oxidation state metal complex), but in NMRP it mostly depends on the persistent radical. It depends on the transfer reagent in RAFT.

An ATRP system may need a particular initiator, other than conventional radical initiators (peroxides and diazo compounds). Unlike ATRP, both NMRP and RAFT always need conventional radical initiators. ATRP is mediated by a transition metal complex which requires ligands for stability. Initiators are usually haloalkanes with a halogen atom usually on secondary or tertiary carbons. Usually a higher oxidation state metal complex acts as a persistent radical. Whereas, NMRP system is mediated by nitroxide radicals which are considered as persistent radicals, and RAFT is mediated by transfer agents (usually dithio compounds) which are not persistent radicals. Halogen capped chains, alkoxyamines and substituted dithio compounds are the dormant species in ATRP, NMRP and RAFT, respectively. Besides, some ATRP systems employ a reducing agents. Ligands and reducing agents are not found in NMRP and RAFT (Braunecker and Matyjaszewski, 2007).

Transition metal complex (mostly copper compounds) used in ATRP is toxic which is the major problem of ATRP. Also, sulphur compounds (as transfer agent) used in most RAFT are also considered as its drawback. The relatively high bond dissociation energy of alkoxyamine (dormant species) needs higher temperature which is the major problem of NMRP. The range of polymerizable monomers is the highest in RAFT followed by ATRP and then NMRP. Monomers with acidic functional groups are difficult to polymerize by ATRP and methacrylate 
monomers are difficult to polymerize by NMRP. One good advantage of ATRP is that it can produce clean block copolymers which is not possible in RAFT. Further, NMRP and RAFT involve purely organic systems, however, moderators are relatively expensive and are required in stoichiometric amounts relative to the number of polymer chains. Furthermore, it is difficult to introduce chain end functionality in NMRP and RAFT as compared to ATRP. Higher commercial availability and more choice of reagents in ATRP are the distinct advantages of ATRP over NMRP and RAFT. Summary of comparison between ATRP, NMRP and RAFT are outlined in Table 2.1. 
Table 2.1: Comparison of ATRP, NMRP and RAFT.

\begin{tabular}{|c|c|c|}
\hline ATRP & NMRP & RAFT \\
\hline $\begin{array}{l}\text { Control in ATRP is achieved } \\
\text { with dynamic chemical } \\
\text { equilibrium between dormant } \\
\text { halogenated radical and } \\
\text { actively propagating radicals. }\end{array}$ & $\begin{array}{l}\text { Control in NMRP is achieved } \\
\text { with dynamic chemical } \\
\text { equilibrium between dormant } \\
\text { alkoxyamines and actively } \\
\text { propagating radicals. }\end{array}$ & $\begin{array}{l}\text { Control in RAFT is achieved } \\
\text { with dynamic chemical } \\
\text { equilibrium of chain transfer } \\
\text { between propagating radicals. }\end{array}$ \\
\hline $\begin{array}{l}\text { Reagents (metal } \\
\text { compounds/ligands/reducing } \\
\text { agents/initiators) are } \\
\text { numerous and commercially } \\
\text { available. }\end{array}$ & $\begin{array}{l}\text { Reagents (especially } \\
\text { nitroxides) are few and } \\
\text { commercially less available. }\end{array}$ & $\begin{array}{l}\text { Less choice for transfer agents } \\
\text { and their lack of commercial } \\
\text { availability. }\end{array}$ \\
\hline $\begin{array}{l}\text { Vast majority of monomers } \\
\text { can be polymerized. Acidic } \\
\text { monomers are difficult to } \\
\text { polymerize. }\end{array}$ & $\begin{array}{l}\text { Range of polymerizable } \\
\text { monomers are less. For } \\
\text { example, methacrylates are } \\
\text { difficult to polymerize. }\end{array}$ & $\begin{array}{l}\text { It can polymerize almost all } \\
\text { monomers that are } \\
\text { polymerizable by FRP. }\end{array}$ \\
\hline $\begin{array}{l}\text { Steady state of growing } \\
\text { radicals are established } \\
\text { through the activation } \\
\text { deactivation equilibrium. } \\
\text { High perturbations in FRP } \\
\text { kinetics. }\end{array}$ & $\begin{array}{l}\text { Steady state of growing } \\
\text { radicals is established through } \\
\text { the activation-deactivation } \\
\text { process. High perturbations in } \\
\text { FRP kinetics. }\end{array}$ & $\begin{array}{l}\text { Steady state radical } \\
\text { concentration is established } \\
\text { through initiation/termination. } \\
\text { It offers minimum } \\
\text { perturbations to FRP kinetics. }\end{array}$ \\
\hline $\begin{array}{l}\text { Does not require continuous } \\
\text { supply of new initiating } \\
\text { radicals. }\end{array}$ & $\begin{array}{l}\text { Does not require continuous } \\
\text { supply of new initiating } \\
\text { radicals. }\end{array}$ & $\begin{array}{l}\text { Requires continuous supply of } \\
\text { new initiating radicals. }\end{array}$ \\
\hline Negligible cross termination. & Negligible cross termination. & $\begin{array}{l}\text { Significant cross termination } \\
\text { between long and short or } \\
\text { newly initiated chains. }\end{array}$ \\
\hline $\begin{array}{l}\text { Pure block copolymers can } \\
\text { be prepared. }\end{array}$ & $\begin{array}{l}\text { Pure block copolymers can be } \\
\text { prepared. }\end{array}$ & $\begin{array}{l}\text { Hard to prepare pure block } \\
\text { copolymers. }\end{array}$ \\
\hline
\end{tabular}




\subsubsection{Reasons for Selecting ATRP over NMRP and RAFT}

The ATRP can be carried out at a milder temperatures than NMRP (Braunecker and Matyjaszewski, 2007; Matyjaszewski et al., 2012; Nabifar, 2007). A higher range of monomers are polymerizable by ATRP than by NMRP (Matyjaszewski, 2003). Disubstituted alkenes (like methyl methacrylates) are difficult to polymerize by NMRP but not by ATRP (Bertin et al., 2004; Chauvin et al., 2006).

The ATRP allows the production of clean block copolymers which is not possible for RAFT. The ATRP does not involve unwanted sulphur chemistry which is essential in most RAFT methods. End group functionalization in ATRP is much easier and almost perfect as compared to RAFT and NMRP. There is a broader choice of reagents in ATRP than in NMRP and RAFT. Reagents are much cheaper and commercially more available in ATRP than in NMRP and RAFT.

However, ATRP has limitations too; such as polymerization in aqueous and protic media is not easy to carry out. Monomers with transferable hydrogen atoms (i.e., acidic monomers like vinyl acetate) are difficult to polymerize. The stoichiometric need of mildly toxic transition metal complex (most often copper) is economically and environmentally unfavourable.

Fortunately, different ATRP methods have been developed to overcome these shortcomings. In particular, ARGET ATRP and ICAR ATRP reduce the catalyst concentration to ppm level, which makes the removal of metal complex from the polymer unwarranted for most applications. The eATRP can virtually stop and start the polymerization as needed. Photoinduced metal free ATRP have recently been developed and can lead ATRP towards the preparation of polymers for biological, microelectronics and other metal sensitive applications (Treat et al., 2014). Significant improvement in ligands and hence reactivity of metal complex has been done to make polymerization possible in aqueous media. Progress has been made in the polymerization of a number of polar monomers. The number of studies done for different CRP techniques till 2012 is in the order ATRP $>>$ RAFT $>$ NMRP, which indicates that ATRP has more potentiality for success than RAFT or NMRP methods (Braunecker and Matyjaszewski, 2007; Matyjaszewski et al., 2012). 


\subsection{Atom Transfer Radical Polymerization (ATRP)}

\subsubsection{Overview}

Mechanistically, ATRP involves transfer of a halogen atom from a dormant halide species to a transition metal complex forming a free radical which is deactivated quickly by the backtransfer of the halogen atom from the transition metal complex. During the time gap between activation and deactivation, the free radical adds few units of monomer. The control of polymerization depends on an appropriate equilibrium between two oxidation states of the transition metal complex. This equilibrium reaction determines the concentration of radicals, rate of polymerization, rate of termination and polydispersities, which is shown in the following reaction scheme (Equations 2.1-2.11) (Braunecker and Matyjaszewski, 2007; Clark, 2002; Minisci, 1975; Xia et al., 1998): The most common AGET ATRP kinetics mechanism is given next.

Initiation

$$
\begin{aligned}
& M t^{q+1} / L i+\mathrm{RA} \rightarrow M t^{q} / L i+\mathrm{OS} \\
& \mathrm{I}-X+M t^{q} / L i \underset{k_{\text {deact }}^{\prime} \stackrel{k_{\text {act }}^{\prime}}{\longrightarrow}}{\stackrel{\bullet}{\longrightarrow}}+X-M t^{q+1} / L i \\
& I^{\bullet}+M \stackrel{k i}{\rightarrow} R_{1}^{\bullet}
\end{aligned}
$$

Propagation. $\quad$ Where, $\mathrm{n} \geq 1 \quad R_{n}^{\bullet}+M \stackrel{k p}{\longrightarrow} R_{n+1}^{\bullet}$

Termination

$$
\begin{aligned}
& R_{n}^{\bullet}+R_{m}^{\cdot} \stackrel{k t c}{\rightarrow} P_{n+m} \\
& R_{n}^{\bullet}+R_{m}^{\cdot} \stackrel{k t d}{\rightarrow} P_{n}+P_{m}
\end{aligned}
$$

Chain transfer

To monomer

$$
\begin{aligned}
& R_{n}^{\bullet}+M \stackrel{K_{t r}^{m o n}}{\longrightarrow} P_{n}+M^{\bullet} \\
& M^{\bullet}+M \stackrel{K_{1}^{m o n}}{\longrightarrow} \mathrm{R}_{1}^{\bullet}
\end{aligned}
$$

To solvent or agent $\quad R_{n}^{\bullet}+S \stackrel{K_{t r}^{S o l}}{\longrightarrow} P_{n}+S^{\bullet}$ 


$$
S^{\bullet}+M \stackrel{K_{1}^{\text {sol }}}{\longrightarrow} \mathrm{R}_{1}^{\bullet}
$$

Equilibrium

$$
R_{n}^{\bullet}+X-M t^{q+1} / L i \underset{\text { kact }}{\stackrel{\text { kdeact }}{\longrightarrow}} R_{n}-X+M t^{q} / L i
$$

Where $\mathrm{RA}=$ reducing agent, $\mathrm{OS}=$ oxidized species, $I^{\bullet}=$ initiator radical, $\mathrm{M}=$ monomer, $\mathrm{X}=$ halogen, $\mathrm{Mt}^{\mathrm{q}} / \mathrm{Li}=$ transition metal complex in lower oxidation state,

$\mathrm{Mt}^{\mathrm{q}+1} / \mathrm{Li}=$ transition metal complex in higher oxidation state,

$R_{1}^{\bullet}=$ propagating radical of length $1, R_{n}^{\bullet}=$ propagating radical of size $\mathrm{n}$ (where $\mathrm{n} \geq 1$ ), $\mathrm{R}_{\mathrm{n}}-\mathrm{X}=$ halide capped polymer, $\mathrm{S}=$ solvent, $P_{n+m} / P_{n} / P_{m}$ are dead polymers of length $\mathrm{n}+\mathrm{m}, \mathrm{n}$ and $\mathrm{m}$ respectively, $\mathrm{k}_{\text {act }}, \mathrm{k}_{\text {deact}}, \mathrm{k}_{\mathrm{i}}, \mathrm{k}_{\mathrm{p}}, \mathrm{k}_{\mathrm{tc}}, \mathrm{k}_{\mathrm{td}}$ and $\mathrm{k}_{\mathrm{tr}}$ are the rate constants of activation, deactivation, initiation, propagation, termination by combination, termination by disproportionation, and chain transfer, respectively.

Note: longer arrow in equilibrium step shows the direction favored by equilibrium.

Referring to the ATRP kinetics mechanism presented above, the rate of reactions can be expressed as:

Rate of polymerization

$$
\mathrm{R}_{\mathrm{p}}=\frac{-d[M]}{d t}=K_{p}[M]\left[P_{n}^{\cdot}\right]
$$

Rate of activation

$$
\mathrm{R}_{\mathrm{act}}=K_{a c t}\left[P_{n}-X\right]\left[M t^{q} / L i\right]
$$

Rate of deactivation

$$
\mathrm{R}_{\text {deact }}=K_{\text {deact }}\left[P_{n}\right]\left[M t^{q+1} / L i\right]
$$

During ATRP equilibrium, the rate of activation equates with that of deactivation, hence:

$$
\begin{aligned}
& \mathrm{R}_{\mathrm{act}}=\mathrm{R}_{\text {deact }} \\
& K_{\text {act }}\left[P_{n}-X\right]\left[M t^{q} / L i\right]=K_{\text {deact }}\left[P_{n}\right]\left[M t^{q+1} / L i\right]
\end{aligned}
$$

Thus the concentration of live polymer radicals is given by:

$$
\begin{aligned}
& \quad P_{n}=\frac{K_{\text {act }}\left[P_{n}-X\right]\left[M t^{q} / L i\right]}{K_{\text {deact }}\left[M t^{n+1} / L i\right]}=\frac{K_{A T R P}\left[P_{n}-X\right]\left[M t^{q} / L i\right]}{\left[M t^{n+1} / L i\right]} ; \\
& \text { Where: } \quad K_{A T R P}=\frac{K_{\text {act }}}{K_{\text {deact }}}=\frac{\left[P_{n} \cdot\right]\left[M t^{q+1} / L i\right]}{\left[M t^{q} / L i\right]\left[P_{n}-x\right]}
\end{aligned}
$$

Hence, putting Equation (2.8) into (2.3) gives the rate of polymerization below: 


$$
\mathrm{R}_{\mathrm{p}}=\frac{K_{p K_{A T R P}[M]}\left[P_{n}-X\right]\left[M t^{q} / L i\right]}{\left[M t^{q+1} / L i\right]}
$$

The polydispersity can be expressed as (Braunecker and Matyjaszewski, 2007):

$$
\mathrm{PDI}=\frac{M_{w}}{M_{n}}=1+\frac{1}{D P_{n}}+\left(\frac{[R X]_{o} k_{p}}{k_{\text {deact }}\left[M t^{q+1} / L i\right]}\right)\left(\frac{2}{\operatorname{Conv}}-1\right)
$$

Where $\mathrm{Mw}$ and $\mathrm{M}_{\mathrm{n}}$ are the weight and number average molecular weights, respectively, and $[\mathrm{RX}]_{\mathrm{o}}$ is the initiator concentration.

According to Equations (2.10) and (2.11), it can be inferred that:

- Polymerization rate increases with initiator concentration and also depends on the ratio of activator $\left(\left[M t^{q} / L i\right]\right)$ to deactivator concentration $\left(\left[M t^{q+1} / L i\right]\right)$.

- For low PDI, sufficiently high concentration of deactivator and low $\mathrm{k}_{\mathrm{p}} / \mathrm{k}_{\text {deact }}$ ratio is needed. PDI also becomes narrow with increasing conversion.

The degree of control in ATRP is strongly affected by the equilibrium constant $\mathrm{K}_{\text {ATRP. }}$ It is important to mention that $K_{A T R P}$ related to copper based complexes is highly dependent on the ligand.

Out of many transition metals (Ti, Mo, Re, Fe, Ru, Os, Rh, Co, Ni, Pd and $\mathrm{Cu}$ ) employed in ATRP, $\mathrm{Cu}$ complexes have been recognized as the most efficient catalysts for ATRP. Commonly used ligands in ATRP are: Bpy, BPMODA, PMDETA, TPMA, $\mathrm{Me}_{6}$ TREN, DMCBCy, dNbpy and tNtpy. Usually activity of the Cu-ligand complexes increases in the following order of ligands: acryl amine $<$ acryl imine $<$ alkyl imine $<$ alkyl amine $\sim$ pyridine (Matyjaszewski et al., 2001).

\subsubsection{Recent Development in ATRP}

The quest for a simple and robust ATRP technique has been continuously growing since its inception in 1995 (Kato et al., 1995; Wang and Matyjaszewski, 1995). The literature on ATRP is growing in a much faster pace than NMRP and RAFT (Braunecker and Matyjaszewski, 2007; Matyjaszewski et al., 2012).

Even though the standard ATRP system has several advantages, it still has some limitations that are summarized below:

- The system is extremely sensitive to oxidant impurities (since it employs highly reducing $\mathrm{Cu}^{\mathrm{I}}$ catalysts) and requires special handling procedures. 
- The metal catalyst concentration (transition metal complex) needs to be relatively high (otherwise low conversion will be achieved), which is environmentally and economically unfavorable.

- Transition metal (copper) is generally considered mildly toxic and its disposal in massive amounts can have environmental concerns.

To address these limitations, a variety of initiating systems have been developed and are provided in Table A.1 (Appendix A) along with a brief description of the processes and advantages/disadvantages associated with each one of them. By far, the most successful ATRP systems are those that use reducing agents (including electrochemical and photo induced ATRP). The reducing agents can help to scavenge the oxidants in the system and reduce the metal catalyst concentration to ppm. These initiating systems are very promising, as they have significantly reduced the major problems existing in ATRP methods. Metal free ATRP, recently discovered, can have a significant benefit over other techniques as it can completely eliminate the use of toxic transition metals and reducing agents. Its progress is highly anticipated.

The ATRP process provides a route to the production of a variety of different molecular architectures: precisely controlled star (co)polymers, block copolymers, end-functional polymers and graft (co)polymers. Application of ATRP products include bio-composites, hybrid materials, tissue engineering, electronics, oil additives, and sealants. As the relation between molecular structure and the end properties of polymer products is important, the number of applications of ATRP processes is expected to increase drastically. Also, further increases in the applications of the ATRP process are likely due to the use of the new initiating systems, which have significantly reduced the need for metal content in ATRP systems, thus making ATRP products more biocompatible and suitable for electronic devices.

Even though ATRP processes have a promising future in polymer technology, more research is needed, especially chemical kinetics, structure-property correlation, and the industrialization of ATRP processes. 


\subsubsection{Recent Advances of ATRP in Aqueous Dispersed Media}

Aqueous dispersed media is the most diverse and benign means of carrying out polymerizations. Water as an environmental and economical dispersion medium is usually selected for the following reasons:

- Good substitute of volatile organic solvents,

- Excellent heat dissipating medium in polymerization, and

- Low viscosity medium and compartmentalization effect of radicals allows high conversion, fast polymerization and formation of high molecular weight polymers, which is very hard to get in bulk and solution polymerizations.

Emulsion polymerization is one of the most dominant technique of polymer production in industry. Polymerizations in aqueous dispersed media are widely utilized to prepare latex with different particle sizes. Based on the polymer particle size obtained from the polymerization and the nucleation mechanism during polymerization, they are commonly classified as the following (Min and Matyjaszewski, 2009):

$>$ Suspension polymerization

$>$ Dispersion polymerization

$>$ Emulsion polymerization

$>$ Miniemulsion polymerization and

$>$ Microemulsion polymerization

Detailed explanations of each polymerization in aqueous dispersed media listed above are given in appendix B.

With the emergence of CRP (including ATRP), a major portion of research has been focused on applying CRP to aqueous dispersed media. However, because of the multicomponent nature of CRP and their complex reaction mechanisms, it is really challenging to adapt CRP in aqueous dispersed media (Candau et al., 1985; Guyot, 2002; Li, 2012; Li and Matyjaszewski, 2011; Min and Matyjaszewski, 2009; Save et al., 2006; Teo et al., 2014; Wei et al., 2015; Wu et al., 2015; Zetterlund et al., 2008). According to several studies, problems observed during CRP (ATRP) in aqueous dispersed media are: 
- Decreased colloidal stability,

- Wide particle size distribution,

- Loss of control over polymerization, and

- Low initiation efficiency.

\subsubsection{Critical Factors}

The success of ATRP in any of the above mentioned dispersed media depends on the several important factors including careful choice of initiation technique of ATRP, surfactant and ligand.

In ATRP, the surfactant is expected to perform the following roles:

- Trap the initiator and catalyst complex in the oil phase,

- Provide stable dispersed system throughout the polymerization,

- Should not interfere with the equilibrium step between dormant and active species.

Both ionic and non-ionic surfactants have been used for emulsion ATRP. In particular nonionic and cationic surfactants are proven to be successful (Zetterlund et al., 2008). Anionic surfactants are still difficult to deploy owing to their interaction with catalyst complexes. However, there is a recent report indicating the possibility of anionic surfactants in ATRP (Teo et al., 2014). Besides, these conventional surfactants, reactive surfactants (surfmer, inisurf and surfactant-ligand) are also reported to be very successful in ATRP (Li and Matyjaszewski, 2011; Wei et al., 2015; Wu et al., 2015).

The selection of a proper ligand is the most important step for a successful ATRP in emulsion. Ligands not only affect the equilibrium between active and dormant species, but they may also determine the location of catalyst complex in the reaction medium. Usually more hydrophilic $\mathrm{Cu}$ (II) complexes tend to go out of the polymerization loci causing loss of control over the polymerization. Use of hydrophobic ligands with very high affinity towards copper can effectively minimize this side effect. Ligands used most often in emulsion ATRP are: bis(2pyridylmethyl)octadecylamine (BPMODA), 4,4'-di-5-nonyl-2,2'-bipyridine (dNbpy) and 4,4"tris(5-nonyl)-2,2':6',2"-terpyridine (tNtpy) (Min and Matyjaszewski, 2009). 


\subsubsection{Successful Initiation Techniques for ATRP in Aqueous Dispersed Media}

It is very hard to handle oxidatively unstable $\mathrm{Cu}(\mathrm{I})$ complexes in industrial scale. Further, rigorous deoxygenation of aqueous media is hard to achieve in large scale. Hence, normal ATRP in aqueous dispersed media is not a good choice (Li, 2012; Li and Matyjaszewski, 2011; Min, 2008; Min and Matyjaszewski, 2009).

The problem of handling oxidatively unstable $\mathrm{Cu}(\mathrm{I})$ complexes can be avoided by using higher oxidation state $\mathrm{Cu}$ (II) complexes as in reverse ATRP. However, this approach demands large amounts of catalyst which can have environmental repercussions. Further, pure block copolymers cannot be prepared by this method.

SR and NI (simultaneous reverse and normal initiation) techniques can be used to reduce the amount of catalyst complex, and have been shown to be successful in emulsion ATRP. However, the drawback of this method is that it does not allow the formation of pure block copolymers in the system.

Limitations of SR and NI to produce pure block copolymer can be overcome by another initiation technique called Activator Generated by Electron Transfer (AGET). ATRP has enjoyed unprecedented success in aqueous dispersed media with this technique. This technique allows easy handling of oxidatively stable $\mathrm{Cu}$ (II) complex as well as formation of clean block copolymers. Another important feature of this technique is that the polymerization rate can be controlled by altering the amount of reducing agent (mostly ascorbic acid or Tin(II)ethylhexanoate) to be added to the system. AGET ATRP can even allow elimination of the deoxygenation step by adjusting the amount of reducing agent, where excess reducing agent consumes dissolved oxygen and remaining reducing agent reduces $\mathrm{Cu}(\mathrm{II})$ into $\mathrm{Cu}(\mathrm{I})$.

Other initiation techniques of ATRP, such as Initiation for Continuous Activators Regeneration (ICAR) and Activators Regenerated by Electron Transfer (ARGET) though successful in bulk or solution, have not been shown much successful in aqueous dispersed media probably because of the very small amount of catalyst and the potential loss of $\mathrm{Cu}$ (II) complex to the aqueous phase. However, there is a report of successful ARGET ATRP in miniemulsion by the use of reactive surfactant (Li and Matyjaszewski, 2009; Stoffelbach et al., 2008). 


\subsubsection{AGET ATRP in Aqueous Dispersed Media}

As discussed above AGET ATRP came as an improvement of SR and NI technique and is currently the best option available for ATRP in aqueous dispersed media, upon which the remaining discussion is focused.

AGET ATRP in Miniemulsion: As discussed in Appendix B, miniemulsion polymerization needs very high shear force, such as ultra-sonication to generate nanometer size monomer droplets (usually 50-500 nm). Kinetically stable but thermodynamically unstable monomer droplets are prevented from coalescence/Ostwald ripening by a small amount of surfactant/stabilizer added in the system. Droplet nucleation is the predominant method of particle nucleation, where homogeneous and micellar nucleation are negligible (Min and Matyjaszewski, 2009).

Advantages of miniemulsion include compatibility for very hydrophobic monomer, need of less surfactant, less sensitivity towards the amount of initiator, agitation speed and polymerization temperature. In miniemulsion, each droplet acts as a nano-reactor mimicking mini-bulk. Since the components are initially located in the droplets, there is no need of mass transfer, which is pivotal to the success of ATRP in miniemulsion. Besides, the combined advantages of AGET initiation technique and miniemulsion dispersed phase, miniemulsion ATRP is difficult to adapt in the industrial scale as it demands intense mixing with very high shear force which is hard to manage on a large scale.

AGET ATRP in Microemulsion: Microemulsion polymerization is mainly used to prepare polymer nanoparticles with potential applications in drug delivery, micro encapsulation, coating formulation, cleaning formulation, oil recovery and many more. Based on microemulsion, a new technique of emulsion polymerization was recently developed for ATRP which can boost the ATRP in emulsion medium (Min and Matyjaszewski, 2009). Less need for high shear force and good latex stability are distinct advantages of microemulsion ATRP. The monomer-solubilizing micelles behave as nano-reactors during a microemulsion and mass transfer can be ignored as in miniemulsion, if initiation is fast. The need of high amount of surfactant is the main disadvantage of microemulsion. Semi-continuous and differential microemulsion polymerization can prepare polymer latexes with high solid content and small particle size $(10-50 \mathrm{~nm})$. Microemulsion ATRP is mostly used to prepare seed particles for chain extension or copolymerization. Out of various initiation techniques, AGET ATRP was 
found to be the most suitable method in terms of overall control over polymerization (narrow PSD and PDI) and comfort (Li, 2012; Min, 2008; Min et al., 2006a; Min and Matyjaszewski, 2005; Yang, 2014).

AGET ATRP in Emulsion Polymerization: For successful AGET ATRP in aqueous dispersed media very hydrophobic ligands and suitable surfactants (reactive surfactants) are developed. However, for an emulsion polymerization this development can create another problem of mass transfer of highly hydrophobic catalyst complex and initiator from monomer droplets to the polymerizing particles/micelles. The compartmentalization of catalyst complex and initiator in monomer droplets can cause monomer droplet nucleation, a predominant nucleation, and the polymerization becomes more like suspension than emulsion with broad PSD. The compartmentalization of catalyst and initiator in the monomer droplet also results in a low initiation efficiency or an uncontrolled polymerization. If relatively high solid content is targeted, then colloidal stability can become another serious issue. This problem can be solved by carrying out polymerization in miniemulsion, seeded emulsion or in a continuous two-step emulsion procedure (Gaynor et al., 1998; Min et al., 2006a; Min and Matyjaszewski, 2009).

There are several reports related to seeded emulsion ATRP, where nucleation step is already completed and the reactants can be loaded in the seed particles. This technique can avoid the challenges associated with emulsion ATRP. For example, seeded emulsion ATRP of Styrene using PBMA particles generated via miniemulsion ATRP gave excellent control over molecular weight distribution (MWD) and high block efficiency (Okubo et al., 2004).

In the two-step emulsion procedure, particles are seeded (nucleated) in microemulsion and more monomer are added later in the ongoing polymerization to make an emulsion system. This two-step procedure circumvents the problem of mass transfer of catalyst complex and initiator which are already confined inside the polymerizing particles (nucleated micelles) during microemulsion phase. The benefit of the two-step emulsion procedure over miniemulsion lies in the fact that it does not need industrially unfavourable high shear force.

During the two-step emulsion polymerization, when the remaining monomers are fed sometime after initiation in the ongoing microemulsion polymerization, pure monomers diffuse from monomer droplets to the polymerizing particles containing catalyst and growing polymers, mimicking a normal emulsion system. A well-controlled AGET ATRP via two-step emulsion polymerization (low PDI and low PSD) with high initiation efficiency, and narrow molecular 
weight distribution (MWD) was reported for a number of monomers including MMA and BA (Min et al., 2006a).

\subsection{ATRP of MMA in Emulsion Systems:}

Polymethyl methacrylate (PMMA) has a long history since its first production in 1928 by companies like Rohm and Haas, Du Pont and ICI. Commercially, it came out into market in 1933 and was sold as Plexiglas, Lucite and Perspex (Cheng et al., 2010; Stoffelbach et al., 2008; Ulrich, 1993).

Different grades of PMMA are widely used, as coatings in glass and electronics, as dental prostheses, surgical bone cements, as material to patch highways and bridges, as automotive lacquers, ion exchange resins, textile additives, paper coating, fiber optics, aircraft glazing, contact lenses etc. It can copolymerize with ethylene glycol to form hydrogel, which is used, as scaffolds in tissue engineering and drug delivery.

ATRP has been controversially the most popular CRP method and MMA is well established and well-studied monomer with versatile applicability in several areas. Because of these features of ATRP and MMA, there is extensive research focused on ATRP of MMA with overwhelmingly huge number of papers already published and new papers are added so frequently. Review of all of these papers is beyond the scope of this research, and only the most pertinent studies (concerned mostly with AGET ATRP of MMA in aqueous dispersed media) are covered here.

Cheng et al., (2013) synthesized homopolymer of MMA (PMMA, polymethyl methacrylate) using surface active initiator in soap-free AGET ATRP miniemulsion. The authors used gemini surface active initiator as both surfactant and initiator and, $\mathrm{CuBr}_{2} / \mathrm{DMDA}$ as catalyst complex. The polymerization was well controlled and the latex was stable.

Wei et al., (2015) performed emulsion ATRP of MMA using surfactant-ligand as the copper capture agent. The authors claimed that they were able to reduce the loss of catalyst complex in the aqueous phase and were also able to reduce surfactant concentration while maintaining acceptable control over the polymerization with good latex stability. The authors used $\mathrm{CuCl}_{2} / \mathrm{dNbpy}$ as catalyst complex and Brij 98 as surfactant.

Zhou et al., (2011) conducted AGET ATRP of MMA in an ionic liquid base microemulsion using $\mathrm{CuBr}_{2}$ / BPMOA (N-bis(2-pyridylmethyl)octylamine) as catalyst. PMMA particles $\sim 5 \mathrm{~nm}$ 
in diameter were produced. Recovery of catalyst complex in ionic liquid was shown to be possible. Further, AGET ATRP of MMA seeded from purified PMMA from previous steps was carried out.

Yang, (2014) also conducted AGET ATRP of MMA in ionic liquid-based microemulsion using $\mathrm{CuCl}_{2} / \mathrm{HMTA}$ as catalyst, ascorbic acid (AA) as reducing agent and $\mathrm{CCl}_{4}$ as an initiator producing PMMA particles with relatively narrow PDI and fairly stable latex.

He and Pan, (2004) reported the synthesis of PMMA and PS (polystyrene) nanoparticles using differential microemulsion polymerization.

Cheng et al., (2014) reported the AGET ATRP of MMA in miniemulsion using photoactive gemini surfactant as initiator (photoinitiator) and surfactant. Polymerization conducted with $\mathrm{CuBr}_{2} / \mathrm{DMDA}$ as catalyst and ascorbic acid (AA) as reducing agent was reported to be well controlled.

Cheng et al., (2010) reported the AGET and ARGET ATRP of MMA in emulsion using surface-active initiator. Polymerization was reported to be well controlled with good latex stability. The authors used $\mathrm{CuBr}_{2} / \mathrm{BPY}$ as catalyst complex and ascorbic acid as reducing agent.

Min et al., 2009, 2006b; Min and Matyjaszewski, 2005, reported the microemulsion ATRP of MMA using $\mathrm{CuBr}_{2}$ / BPMODA as catalyst complex and Brij 98 as a surfactant. In their study they tested FRP, normal ATRP, reverse ATRP and AGET ATRP initiation techniques and claimed that AGET ATRP was the best in terms of overall control of the polymerization and stability of the latex. In their next paper (Min et al., 2009) the author used exact same AGET ATRP of MMA in microemusion as a first step towards the synthesis of PMMA-PnBA hairy nanoparticles. In a following paper by same author (Min et al., 2006b) successful AGET ATRP in the presence of air in miniemulsion was reported for butyl acrylate.

$\mathrm{Li}$, (2012) in her thesis has reported the AGET ATRP of MMA in microemulsion and emulsion (two step) using $\mathrm{CuBr}_{2}$ /BPMODA as catalyst complex, $\mathrm{CTAB}$ as surfactant, $\mathrm{AA}$ as reducing agent and $\mathrm{EBiB}$ as initiator. Polymerization was reported to be well controlled with good colloidal stability for both microemulsion and two-step emulsion. The author has reported that the $9 \mathrm{wt} \%$ surfactant versus monomer resulted into the multiple peaks in the DLS analysis although the level of control for the polymerization was acceptable. However, this surfactant concentration (9 wt\% vs monomer) is also higher for the industrial applications. 
Li et al., (2015)reported the preparation of PMMA macromonomers via AGET ATRP in emulsion (two-step) system for PnBA-g-PMMA copolymerization using $\mathrm{CuBr}_{2} / \mathrm{dNbpy}$ as catalyst, Brij 98 as surfactant, $\mathrm{EBiB}$ as initiator and AA as reducing agent. In their report, the authors mentioned the use of $\mathrm{NaCl}$ along with surfactant for better latex stability. According to the authors the polymerization was well controlled with good latex stability, however the conversion was still low (63.4\%) even after $10 \mathrm{hrs}$ and they used industrially unfavourable amount of surfactant (around $32 \mathrm{wt} \%$ vs monomer).

Wei et al., (2014) reported the single step emulsion AGET ATRP of BMA using CuCl2/dNbpy, VA-044, EBiB, AA, SL (surfactant ligand) and Tween 80, followed by chain extension with MMA in solution ATRP. The authors reported uncontrolled polymerization while using water soluble initiator VA-044. However, while using oil soluble initiator EBiB, relatively broad PDI $(1.69,1.56$ and 1.49) was reported based on the amount of dNbpy in the system.

To our best knowledge to date, only one published paper ( $\mathrm{Li}$ et al., 2015) synthesized PMMA by two-step emulsion AGET ATRP of MMA using $\mathrm{CuBr}_{2} / \mathrm{dNb}$ y as catalyst complex, Brij 98 as surfactant, $\mathrm{AA}$ as reducing agent and $\mathrm{EBiB}$ as initiator. In their paper, the authors also reported the use of $\mathrm{NaCl}$ for better latex stability.

As summarized in section 1.2, the study carried out in this thesis focuses on an experimental investigation of emulsion AGET ATRP polymerization of MMA in a 2-liter reactor system. 


\section{CHAPTER 3}

\section{MATERIALS AND METHODS}

\subsection{Materials}

The polymerization reaction was carried out in a 2-L stainless steel reactor as described in

Section 3.2. The reaction recipe is provided in Table 3.1:

Table 3.1: Reaction materials for emulsion AGET ATRP of MMA

\begin{tabular}{|c|c|c|c|c|}
\hline \multirow[t]{2}{*}{ Reactants } & \multicolumn{3}{|c|}{ Chemical Components } & \multirow{2}{*}{$\begin{array}{l}\text { Quantity } \\
\text { (range) }\end{array}$} \\
\hline & Acronym & Full name & Purity & \\
\hline Monomer* & MMA & Methyl Methacrylate & $\begin{array}{lr}99 \%(\leq 30 & \mathrm{ppm} \\
\text { MEHQ } & \text { as } \\
\text { inhibitor }) & \\
\end{array}$ & $27.41-98.42 \mathrm{~g}$ \\
\hline Initiator** & EBiB & Ethyl-2-bromoisobutyrate & $>98 \%$ & $0.2374-0.9702 \mathrm{~g}$ \\
\hline Catalyst** & $\mathrm{CuBr}_{2}$ & Copper(II)bromide & $99 \%$ & $0.0113-0.1006 \mathrm{~g}$ \\
\hline Ligand* & dNbpy & 4, 4'-Dinonyl-2, 2'-dipyridyl & $97 \%$ & $0.0725-0.3678 \mathrm{~g}$ \\
\hline Surfactant* & Brij 98 & $\begin{array}{l}\text { Polyoxyethylene (20) oleyl } \\
\text { ether }\end{array}$ & Not determined & $5.4671-18 \mathrm{~g}$ \\
\hline Reducing agent* & $\mathrm{AA}$ & Ascorbic acid & $99 \%$ & $0.0138-0.0862 \mathrm{~g}$ \\
\hline $\begin{array}{l}\text { Dispersion } \\
\text { medium }\end{array}$ & Water & Water & Distilled & $348.95-598.2 \mathrm{~g}$ \\
\hline $\begin{array}{l}\text { Polymer } \\
\text { precipitant** }\end{array}$ & Methanol & Methanol & $99.80 \%$ & N/A \\
\hline GPC solvent* & THF & Tetra Hydro Furan & $\begin{array}{l}\geq 99.9 \% \\
\text { HPLC grade }\end{array}$ & N/A \\
\hline
\end{tabular}

\footnotetext{
**purchased from VWR (Canada)

* purchased from Sigma Aldrich (Canada)
}

The monomer was purified by passing it through a specified column, containing inhibitor remover $\mathrm{Al}_{2} \mathrm{O}_{3}$, to remove the inhibitor mono methyl ether hydroquinone (MEHQ) . The column was purchased from Sigma Aldrich (Canada). The purified monomer was put in a dark brown reagent bottle and stored at $0-2^{\circ} \mathrm{C}$. All other chemicals were used as received. Narrow and broad standard polystyrene samples for GPC analysis were purchased from PolyAnalytik (London, Ontario, Canada). In general, the reaction was carried out at constant temperature ranging from $50^{\circ} \mathrm{C}$ to $70^{\circ} \mathrm{C}$ and reaction medium was continuously stirred at 150 to $250 \mathrm{rpm}$. 


\subsection{Reactor Setup}

The polymerization was performed in a 2-L stainless steel PARR reactor vessel (series 4530) connected to a temperature controller heating/cooling bath (Cole-Parmer Polystat H28L). A detailed schematic diagram of the reactor system is provided in Figure 3.1. The reactor is equipped with a temperature control unit (series 4848) and impeller speed controller. The reactor vessel is a flat-bottomed cylindrical tank with a diameter of $10.16 \mathrm{~cm}$ and a height of $26.67 \mathrm{~cm}$. It is equipped with $45^{\circ}$ pitched-blade turbine of a $5 \mathrm{~cm}$ diameter. The power of the stirring motor is $1 / 4 \mathrm{hp}$. The off-bottom clearance is $1.3 \mathrm{~cm}$. Besides, a U-shaped cooling coil connected to the circulator bath. The reactor temperature was measured using type J thermocouples. An inlet and outlet pipe for injection of chemicals, sampling and gas purging was inserted in the reactor. Prior to starting the actual experiment, the reactor is usually, run with distilled water for few hours. During this period, the reactor auto-tuning is performed, where assessment of the control of the heater and reactor temperatures, motor speed and reactor pressure is achieved.

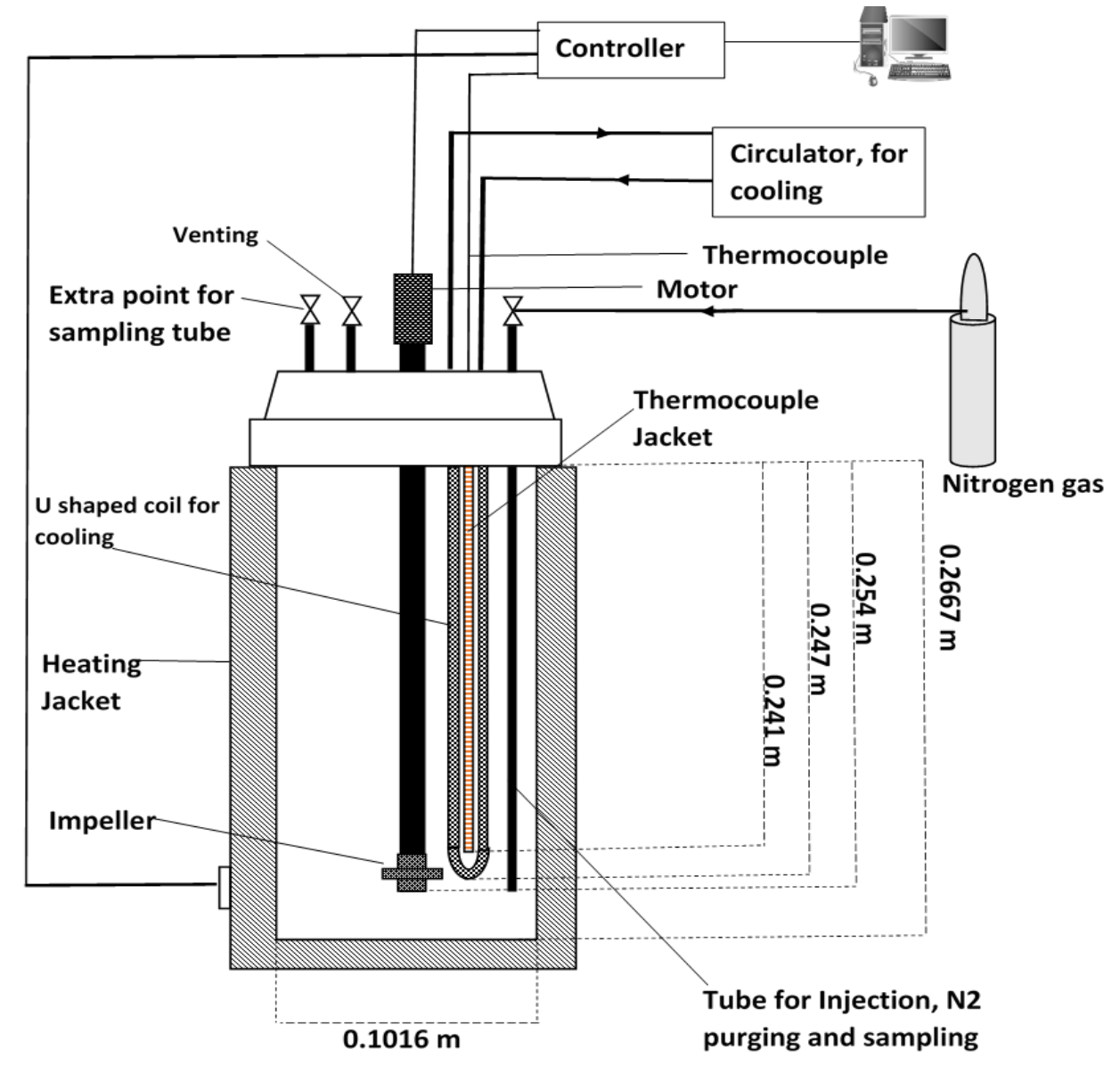

Figure 3.1: Schematic diagram of reactor set up. 


\subsection{Experimental Procedure}

Two experimental procedures were performed in this study.

\section{a) Single-Step Emulsion}

First, the catalyst $\mathrm{CuBr}_{2}$ and the ligand dNbpy were dissolved in MMA to form a solution. Then, the initiator (EBiB) was dissolved in the MMA solution, which was poured slowly into an aqueous solution of Brij 98 under stirring. A milky white emulsion was formed. The whole mixture was transferred into the reactor and purged with nitrogen. Then, a solution of ascorbic acid (AA) in distilled water was injected into the reactor at the selected temperature to activate the catalyst and start the polymerization. Samples were withdrawn at selected time periods for analysis and characterization of the polymer. Each aliquot after withdrawal was shaken with air trapped inside the vial and then cooled to stop the polymerization.

\section{b) Two-Step Emulsion}

The procedure for two-step emulsion system differs from single-step emulsion procedure in that the polymerization was initiated in microemulsion at first followed by the injection of additional monomer on the ongoing microemulsion polymerization after some time of initiation, to form an emulsion system.

The catalyst $\mathrm{CuBr}_{2}$ and ligand dNbpy were dissolved in MMA to form a solution of the catalyst complex in which EBiB was dissolved. Meanwhile, the surfactant Brij 98 was dissolved in water. The organic MMA solution, containing catalyst complex and initiator, was poured slowly into the aqueous solution of Brij 98 under stirring to form an optically translucent/transparent microemulsion. The whole mixture was transferred into the reactor and purged with nitrogen. An aqueous solution of ascorbic acid (AA) in distilled water was injected into the reactor at the set temperature to activate the catalyst and start the polymerization. After some time of initiation, the second portion of MMA was added to the ongoing microemulsion polymerization to form an emulsion polymerization. The polymerization was carried out under the inert atmosphere of nitrogen gas. Samples were withdrawn at the designated time to measure conversion gravimetrically and to determine molecular weight and molecular weight distribution by GPC. Each aliquot after withdrawal was shaken with air trapped inside the vial and then cooled to stop the polymerization. 


\subsection{Polymer Characterization}

\subsubsection{Gravimetry}

The extent of monomer conversion was determined by off-line gravimetry. An aliquot of each sample (1-2 mL) was weighed in a dry and clean aluminum cup and dried in a vacuum oven at $45-50^{\circ} \mathrm{C}$ for $24 \mathrm{~h}$ under $620 \mathrm{~mm}$ of $\mathrm{Hg}$ vacuum pressure (actual pressure $140 \mathrm{~mm}$ of $\mathrm{Hg}$ ). Water and monomer were therefore evaporated. The remaining solid is mainly poly-MMA and some traces of other solids (catalyst complex, surfactant, initiator and reducing agent). The cup with the dry residue was weighed again. The weight fraction of solids is calculated as follows (Penlidis, 1986):

$W . f$. solids $=\frac{(\text { weight of cup }+ \text { dry residue })-(\text { weight of cup })}{(\text { weight of cup }+ \text { wet sample })-(\text { weight of cup })}$

From the formulation of the reaction, the weight fraction of each component was calculated and then the polymer conversion was calculated by using the following formula.

$x(t)=\frac{w \cdot f \cdot s o l i d s-(w . f . i n i t i a t o r+w . f . c a t a l y s t \text { complex }+w \cdot f \cdot s u r f a c t a n t+w \cdot f \cdot r e d u c i n g \text { agent })}{w \cdot f \cdot \text { monomer }}$

Where, $x(t)$ is the weight fraction of PMMA.

\subsubsection{Gel Permeation Chromatography (GPC)}

Size exclusion chromatography (SEC) also referred to as gel permeation chromatography (GPC) is one of the most popular and convenient method to determine average molecular weights $\left(\mathrm{M}_{\mathrm{n}}, \mathrm{M}_{\mathrm{w}}\right)$ and molecular weight distribution (MWD).

Measurement of the molecular weights of the polymer samples collected in this study were performed using GPC (Viscotek TDA, Model 302) equipped with a triple detector array in which tetrahydrofuran (THF) was used as the mobile phase at a nominal rate of $1.0 \mathrm{~mL} / \mathrm{min}$. The characteristic system consists of a Viscotek GPC max VE 2001 GPC solvent/sample module as auto sampler followed by Viscotek's triple detector equipped with a low- and rightangle laser light scattering detectors (LALLS/RALLS), differential refractometer (RI) and viscometer in series. Detector and column temperatures were maintained at $22^{\circ} \mathrm{C}$ and laser detector operated at $670 \mathrm{~nm}$. The number average molecular weights $\left(\mathrm{M}_{\mathrm{n}}\right)$ and polydispersity $\left(\mathrm{Mw} / \mathrm{M}_{\mathrm{n}}\right)$ were determined by means of a calibration curve of polystyrene standards. The 
injection volume varied between 100 and $60 \mu \mathrm{L}$. A specific refractive index increment $(\mathrm{dn} / \mathrm{dc})$ value of $0.185 \mathrm{~mL} / \mathrm{g}$ was used in the light scattering analysis for standard polystyrene (PS).

The polymer, precipitated from latex by using excess of methanol was dissolved in THF and then precipitated back in 50:50 $\mathrm{CH}_{3} \mathrm{OH}: \mathrm{H}_{2} \mathrm{O}$ followed by washing with water and 50:50 $\mathrm{CH}_{3} \mathrm{OH}: \mathrm{H}_{2} \mathrm{O}$. Then, it was dried in the vacuum oven at $45-50^{\circ} \mathrm{C}$ for 24 hours. Then, a precise weight of dried polymer was dissolved in a fixed amount of HPLC grade THF. Prior to injection, polymer solutions were filtered through a $0.20 \mu \mathrm{m}$ filter to remove any insoluble gels.

\subsubsection{Reliability of Measurements}

Different sources of errors that are expected to occur in an experimental work were kept to a low level. They may come from measurements, the preparation of solutions and deficiency in device accuracy were minimized as follows:

- Calibration of the GPC column was done with narrow standard polystyrene. After calibration, a blank run (pure THF only) was analyzed to make sure that the column did not contain any residual substance from previous analysis, then a broad standard polystyrene was also analyzed to assess the reliability of the analysis. Once the GPC machine was calibrated and standardized, then analysis of each sample was done with at least 3 replicates. Fairly close value of measurements from each replicate revealed the reliability of the GPC machine. Further, some of the samples were analyzed at the Department of Chemical Engineering, University of Waterloo and compared with the results of GPC at Ryerson University. Fairly close value of PDI and Molecular weight results from both GPC machines further proved the reliability of the measurements.

- For conversion determination, the gravimetry was replicated for some of the samples with different initial amount of latex. Almost same result obtained each time confirmed the reliability of the gravimetry device.

- A scale (Meter Toledo AB 104-S) of high sensitivity was used to get precise weighing of the chemicals. Calibration was done by adjusting the measurement for an internal weight. The AB-S certified models have an internal weight that adjusts themselves automatically, twice within $2 \mathrm{~h}$ of connection to the power supply. 


\subsection{Experimental Design and Statistical Method}

Design of experiments (DOE) is a systematic approach that applies statistical principles and techniques at the data collection stage in order to ensure the generation of valid, reliable and supportable conclusions. In general, the experimental design refers to the process of planning, designing and analyzing experiments so that valid and sound conclusions can be drawn effectively and efficiently.

In order to achieve such an objective, it is necessary to integrate simple and powerful statistical methods into the experimental design methodology. Besides, the design of experiments reveals the effect of each process variable or parameter and its interactions on the process output response and the overall process behaviour. It also helps to develop input-output models relating the input variables to process responses (Alam et al., 2008; Lazić, 2004).

\subsubsection{Two Level Fractional Factorial Design}

The classical approach of changing one factor at a time to study its effect on the process response (dependent variable) is a time-consuming procedure for multivariable systems. Another major disadvantage of this scheme is that it fails to reveal any possible interaction between the factors. More importantly, the statistical design of experiments helps reducing the number of experiments (Montgomery, 2012; Natrella, 2010).

One plausible approach to analyze and model multivariable processes is to use a fractional factorial design of experiment, in which independent variables are varied together, instead of one at a time. A screening design approach is usually utilized to determine the variables, that have the highest correlations. In particular, the two-level factorial design methodology is a tool for this purpose, principally because they are efficient and economical (Kalil et al., 2000). This design helps to evaluate factors and select the most important ones.

As the number of factors in two-level factorial designs increases, the number of runs becomes even larger, because the total number of experiments for a full two-level factorial design increases exponentially with the number of factors $\left(2^{\mathrm{K}}\right)$. Hence, the full factorial design becomes infeasible taking into account the available resources and experimental time, 
especially for $\mathrm{K} \geq 5$. Fractional factorial designs (FFDs) can be used in such cases to draw valuable results from fewer runs.

Fractional factorial design is a variation of the full factorial design in which only a subset of the runs is made which reduces the number of actual experiments to be performed. Fractional factorial experiments are more useful when there are several variables and the process under study is expected to be primarily governed by some of the main effects and lower order interactions (Toutenburg and Shalabh, 2009).

Efficiency and reliability of estimation of effects using fractional factorial design depends upon its resolution. Design resolution is a summary characteristic of confounding patterns. It is a key tool for determining what fractional factorial design will be the best choice for a given problem. The term 'confounding' refers to the combining influences of two or more factor effects in one measured effect, in other words, one cannot estimate factor effects and their interaction effects independently.

Usually, low-resolution designs are used for screening among main effects, whereas higher resolution designs are deployed if interaction effects and response surfaces need to be investigated. Although fractional factorial designs (FFDs) avoid a large number of runs of full factorial designs, it suffers from the confounding (alias) effects which makes main effect estimates from fractional factorial not as good as full factorial, which is the price to pay for using fewer runs. In case interactions between factors are strong, then fractional factorial design with low resolution may not be a reasonable choice.

Designs of resolution three, and sometimes four are usually preferred for screening purpose, as they require fewer runs. We refer to a design as a screening design (or main effect design) if its primary purpose is to identify significant main effects, rather than interaction effects. On the other hand, designs of resolution five and higher are used for focusing on more than just main effects in an experimental situation. These designs allow us to estimate interaction effects and they can be easily augmented to complete a second-order design - a design that permits estimation of a full second-order (quadratic) model (Natrella, 2010).

In resolution 5 designs, the main effects are not confounded/aliased with other main effects, two factor interactions or three-factor interactions. However, main effects would be confounded with four-factor (and possibly higher-order) interactions and two-factor interactions would be confounded with certain three-factor interactions. Higher-order 
interactions are less likely to be significant than low-order interactions. If the desired effects are only confounded with non-significant interactions, then the model is usually acceptable.

The chosen design should be completely randomized if possible, to reduce the effect of experimental bias. The purpose of randomisation is to remove all sources of external variations which are uncontrollable disturbances in real-life settings. By properly randomising an experiment, one can assist in averaging out the effects of noise factors undesirably associated with the process. Randomization can ensure that all levels of a factor have an equal chance of being affected by noise factors.

The fractional factorial method has the following consecutive steps:

1. Selecting process parameters and their ranges,

2. Designing the experiments and conducting them,

3. Generating a modeling scheme using linear regression based on the experimental results, and

4. Testing the accuracy of the developed model.

\subsection{Experimental Design Selection}

Various experimental designs are available for conducting the experiments, they differ from one another in terms of their resolution, the number of runs and confounding patterns.

Five factor two-level fractional factorial design with resolution five $\left(2_{\mathrm{v}}{ }^{5-1}\right)$ is of particular interest and it was chosen to model the AGET ATRP of MMA polymerization in the stirred 2$\mathrm{L}$ batch reactor. It is a very powerful design which allows unique estimation of all the main effects and two-factor interactions, provided that all the three factor and higher interactions are negligible. The strength of this design also lies in the fact that, even though individual quadratic effects cannot be estimated, they can still be detected efficiently, when center point runs are included with experimental runs.

\subsubsection{Selection of Process Variables}

Preliminary experimental investigation was conducted to select the independent variables of AGET ATRP polymerization process. The process output variables were defined in the 
objectives of the study. In this polymerization process, monomer (MMA) conversion and polymer molecular weight averages are measurable, and they were selected as the process output variables because they definitely characterize the polymer produced. The independent variables, temperature, catalyst complex, reducing agent, surfactant and initiator are the input process variables which can have the most important influence on the quality and structure of the end product (polymer). The experimental runs were performed at two levels: low and high, however, center levels were also included for center point runs, which provided a measure of process stability and inherent variability. Center point runs can also detect the curvature in the model of individual response variables. Generally, 3-5 center point runs are recommended in the FFD experimental design. Center level is taken midway level between the low and high levels of the variable. This procedure was applied to each variable independently.

\subsubsection{Selection of the Model}

Once the experiments have been conducted according to the chosen experimental design, the results are employed to develop a functional relationship between the key independent and response variables of the process. The statistical method of analysis expresses the process response (dependent variable) as a function of the experimental (independent) variables and the interactions between the variables.

For the fractional factorial design procedure, it is necessary to know the alias structure in order to write an appropriate starting model containing only the interaction terms the experiment was designed to estimate (assuming all terms confounded with these selected terms are insignificant). Usually, all main effect and interaction terms are included initially to construct the normal (or half-normal) plots of effects, but later some of the main effects and interaction terms can be dropped to get a simpler, adequate model fit. Various techniques such as normal or half-normal plots, $p$-value comparisons and stepwise regression routines can be used to reduce the model to the minimum number terms required.

First-order model is generally used for the main effects only. However, for the interaction between the variables, the experimental data should be fitted to a second-order equation that contains terms representing main effects and second-order interactions as follows. Higherorder interactions are supposed to be insignificant and are excluded from the model. 


$$
y=b_{o}+\sum_{i=1}^{n} b_{i} x_{i}+\sum_{i=1}^{n-1} \sum_{j=2}^{n} b_{i j} x_{i} x_{j}
$$

Where $\mathrm{y}$ is the process output (dependent variable), $\mathrm{n}$ is the number of factors (independent variables), $b_{o}$ is the regression coefficient at the intercept, and $b_{i}$ and $b_{i j}$ are the regression coefficients for the linear and interaction effects of each factor $x_{i}$ respectively.

The model prediction results are generally presented graphically as 2-D and 3-D plots, which helps in visualizing a better interpretation of the functional relationships between the response and independent variables. The model can provide information about the system behaviour at different conditions but cannot explain the mechanism of the process.

\subsubsection{Statistical Method for Confirmation of the Model Accuracy}

The developed model has to be tested to ensure an adequate prediction of the system (polymerization process) behaviour. The statistical validation is determined by the analysis of variance (ANOVA) test at $95 \%$ confidence level. The $p$-value is the probability of null hypothesis being true in the observed result. Since $p$-value $<0.05$ is taken as a threshold value, it indicates that the probability value used to set the confidence level is at $95 \%$. Hence the lower the $p$-value $(\leq 0.05)$ more significant will be the key variable. The quality of the multivariable polynomial model is usually explained by coefficient of determination $\left(\mathrm{R}^{2}\right)$ which shows the percentage of variation in the response from the mean that has been explained by the fitted model. For a model with good prediction efficiency, the value of $\mathrm{R}^{2}$ should be close to 1 . However, $\mathrm{R}^{2}$ alone is not the criteria to assess the model, since it increases with the increase in the number of terms in the model equation. Another way of measuring the adequacy of the model is to compare $\mathrm{R}^{2}$ value with $\mathrm{R}^{2}$ adjusted value, where $\mathrm{R}^{2}$ adjusted value decreases if statistically insignificant process variables are selected. The closer the values of $\mathrm{R}^{2}$ and $\mathrm{R}^{2}$ adjusted, the better the model adequacy. Residual analysis is another important aspect to confirm the model adequacy, where residuals are the difference between the predicted and actual values which assesses whether the observed error (residuals) is consistent with stochastic error. The predicted values are compared with experimental data to validate the adequacy of the developed model. 


\section{CHAPTER 4}

\section{PRELIMINARY EXPERIMENTAL INVESTIGATION OF AGET ATRP OF MMA EMULSION POLYMERIZATION}

\subsection{Introduction}

Atom transfer radical polymerization (ATRP) is a very popular CRP method, which has been quite successful in the preparation of diverse and well-controlled polymeric materials. Distinct advantages of ATRP over other methods of CRP includes mild reaction temperature, wide range of monomers (including methacrylates), easier end group functionalization and easier clean block copolymer production (Ayres, 2011; Barner-Kowollik et al., 2002; Bertin et al., 2004; Chan et al., 2013; Chauvin et al., 2006; Coessens and Matyjaszewski, 2010; Matyjaszewski, 2012, 2003; Matyjaszewski et al., 2012; Matyjaszewski and Xia, 2001; Nabifar, 2007; Pintauer and Matyjaszewski, 2008).

Even though ATRP in bulk and solution has been extensively studied, not much success was achieved in aqueous dispersed media. For economic and environmental reasons, conducting polymerization in aqueous dispersed media is industrially more attractive. In fact, emulsion polymerization is very popular in the polymer industry (Oh, 2008); Min et al., 2006a (Min et al., 2006a).

It is really challenging to adapt ATRP in aqueous dispersed media (Eslami and Zhu, 2006; Oh, 2008). Problems mostly observed during ATRP in aqueous dispersed media are related to: decreased colloidal stability, wide particle size distribution, loss of control over polymerization and low initiation efficiency (Eslami and Zhu, 2005; Min, 2008; Min and Matyjaszewski, 2009; Oh, 2008; Save et al., 2006; Wei et al., 2015).

Despite several challenges, little success of ATRP in aqueous dispersed media is mainly due to the new initiation technique known as AGET ATRP (Min et al., 2009, 2006b; Min and Matyjaszewski, 2005).

It is important to report that the chemistry and kinetic/mechanistic aspects of ATRP in emulsion are still not well understood (Eslami and Zhu, 2006). There is no detailed or reliable 
experimental study conducted over a range of reaction conditions to validate and support the development of reaction mechanisms.

In this study, AGET ATRP of MMA with a limited amount of air is explored in single-step emulsion and two-step emulsion in a 2 liter stirred tank reactor using commercially available chemicals: copper bromide/4,4'-Dinonyl-2,2'-dipyridyl $\left(\mathrm{CuBr}_{2} / \mathrm{dNbpy}\right)$ as catalyst complex, polyoxyethylene (20) oleyl ether (Brij 98) as surfactant. Ascorbic acid (AA) and ethyl-2bromoisobutyrate $(\mathrm{EBiB})$ are used as reducing agent and initiator, respectively. To polymerize fairly hydrophilic monomer MMA $\left(15.3 \mathrm{~g} / \mathrm{L}\right.$ at $\left.20^{\circ} \mathrm{C}\right)$ in an aqueous phase, a partially soluble surfactant is used to stabilize the reaction. Its primary role is to lower the surface tension allowing the emulsification of MMA and formation of stable colloidal dispersion of polymer particles. A hydrophobic initiator, used in the reaction, generates free radicals by interaction with a metal complex and causes MMA to polymerize. The aim of the study is to investigate the performance of single-step and two-step emulsion polymerization systems to produce PMMA and use it as a basis for the selection of process parameters for the design of experiments and statistical analysis.

\subsection{AGET ATRP Experimental Procedures and Results}

The materials, reactor setup, reactor operation and polymer characterization methods are discussed in chapter 3.

Carrying out AGET ATRP of MMA in an emulsion medium in a 2-L reactor is a quite original study and the literature shows no similar investigation on this kind of polymerization.

There are no obvious guidelines to choose the experimental recipes and therefore, the first few experiments served to determine the right proportion of reactants and reasonable reaction conditions. Several experimental tests were randomly done and did not produce good results.

The single-step and two-step emulsion polymerization experimental procedures that were conducted in this study are discussed next. 


\subsubsection{Single-Step Emulsion Procedure}

A series of PMMA latexes were synthesized via the single-step polymerization technique. The runs and detailed ratio of reagents are listed in Tables 4.1 and 4.2. The reactions were carried out at two temperatures, 50 and $70^{\circ} \mathrm{C}$, and a reaction time up to $5.5 \mathrm{~h}$ for some experiments. In a typical experiment (e.g., Run S6), $\mathrm{CuBr}_{2}(0.0525 \mathrm{~g})$ and dNbpy (0.1726 g) were dissolved in MMA (46.8 g) to form a solution with the catalyst complex. The initiator (EBiB, $0.4067 \mathrm{~g}$ ) was dissolved in the solution of catalyst complex. The solution of Brij $98(9.39 \mathrm{~g})$ was prepared in $590 \mathrm{~mL}$ distilled water, which was transferred to the reactor, then the stirrer was turned on and the solution was purged continuously with nitrogen gas for 3 minutes. The solution of catalyst complex along with initiator was injected into the reactor using a glass syringe to make emulsion. Then, the solution of ascorbic acid (AA, 0.045g) in distilled water $(10 \mathrm{~mL})$ was injected into the reactor at the desired temperature of $50^{\circ} \mathrm{C}$ to activate the catalyst and start the polymerization. Polymer samples were withdrawn at selected time periods for analysis and characterization. Each aliquot after withdrawal was shaken with air trapped inside the vial and then cooled down to stop the polymerization.

\subsubsection{Results and Discussion of Single-Step Emulsion Procedure:}

Seven experimental tests were performed under different experimental conditions as shown in Tables 4.1 and 4.2 .

Table 4.1: Recipe for the single-step emulsion AGET ATRP of MMA.

\begin{tabular}{|c|c|c|c|c|c|c|c|c|}
\hline Expt. & $\begin{array}{l}\text { EBiB } \\
(g)\end{array}$ & $\begin{array}{l}\text { Brij } \\
98(g)\end{array}$ & $\begin{array}{l}\text { MMA } \\
\text { (g) }\end{array}$ & $\begin{array}{l}\text { dNbpy } \\
\text { (g) }\end{array}$ & $\begin{array}{l}\text { Ascorbic } \\
\text { acid (g) }\end{array}$ & $\begin{array}{l}\mathrm{CuBr}_{2} \\
\text { (g) }\end{array}$ & $\begin{array}{l}\text { Water } \\
\text { (g) }\end{array}$ & $\begin{array}{l}\text { Molar ratio: EBiB: Brij98: } \\
\text { MMA: dNbpy: AA: CuBr2 }\end{array}$ \\
\hline S1 & 0.4067 & 9.3900 & 46.8000 & 0.2555 & 0.0222 & 0.0696 & 598.2000 & \multirow[t]{2}{*}{ 1:3.92:224:0.3:0.06:0.15 } \\
\hline $\mathrm{S}^{\mathrm{a}}$ & 0.4067 & 9.3900 & 46.8000 & 0.2555 & 0.0222 & 0.0696 & 598.2000 & \\
\hline S3 & 0.4067 & 9.3900 & 46.8000 & 0.2555 & 0.0440 & 0.0696 & 598.2000 & \multirow[t]{2}{*}{ 1:3.92:224:0.3:0.12:0.15 } \\
\hline S4 & 0.4067 & 9.3900 & 46.8000 & 0.2555 & 0.0440 & 0.0696 & 598.2000 & \\
\hline S5 & 0.4067 & 9.3900 & 46.8000 & 0.1726 & 0.0370 & 0.0525 & 598.2000 & 1:3.92:224:0.2:0.10:0.11 \\
\hline S6 & 0.4067 & 9.3900 & 46.8000 & 0.1726 & 0.0450 & 0.0525 & 598.2000 & 1:3.92:224:0.2:0 \\
\hline S7 & 0.2374 & 5.4671 & 27.4100 & 0.2247 & 0.0339 & 0.0613 & 348.9500 & $1: 3.92: 224: 0.45: 0.16: 0.23$ \\
\hline
\end{tabular}

a more $\mathrm{EBiB}(0.1032 \mathrm{~g})$, AA (0.005g) and MMA (9.36g) were added into the ongoing polymerization after $2.2 \mathrm{~h}$ of initiation. 
It can be noted that experiment S7 has a noticeably different ratio of mass than in the rest of the experiments which is mainly due to the scale down of recipe/total mass of the reactants as some changes in the features of the reactor at that time allowed to do so.

Table 4.2: Experimental conditions for the single-step emulsion AGET ATRP of MMA.

\begin{tabular}{|c|c|c|c|c|c|c|}
\hline Expt. & $\begin{array}{l}\text { Temp } \\
\left({ }^{\circ} \mathrm{C}\right)\end{array}$ & $\begin{array}{l}\text { Pressure of } \\
\text { nitrogen (psig) }\end{array}$ & $\begin{array}{l}\text { Working volume of } \\
\text { the reactor }(\mathrm{mL})\end{array}$ & $\begin{array}{l}\text { Wt \% of Brij } 98 \\
\text { versus MMA }\end{array}$ & $\begin{array}{l}\text { Motor } \\
\text { speed (rpm) }\end{array}$ & $\begin{array}{l}\mathrm{N}_{2} \\
\text { Purging }\end{array}$ \\
\hline S1 & 70 & 20 & 650 & 20 & \multirow{3}{*}{250} & \multirow{2}{*}{$\begin{array}{l}15 \text { min for } \\
\text { whole } \\
\text { emulsion }\end{array}$} \\
\hline S2 & 70 & 20 & 650 & 20 & & \\
\hline S3 & 70 & 20 & 650 & 20 & & \multirow{5}{*}{$\begin{array}{c}3 \text { min for } \\
\text { surfactant } \\
\text { solution } \\
\text { only }\end{array}$} \\
\hline S4 & 70 & 20 & 650 & 20 & 150 & \\
\hline S5 & 50 & 20 & 650 & 20 & \multirow{3}{*}{250} & \\
\hline S6 & 50 & 20 & 650 & 20 & & \\
\hline S7 & 50 & 20 & 380 & 20 & & \\
\hline
\end{tabular}

Referring to Table 4.3 and Figure 4.1, the data show that very low conversion was obtained in the first experiment $\mathrm{S} 1$. Loss of $\mathrm{EBiB}$ and MMA during purging for a long time is likely the cause for the low conversion. For experiment S2, the conversion was suddenly increased after adding more $\mathrm{EBiB}, \mathrm{AA}$ and $\mathrm{MMA}$ in the middle of the reaction (after $2.2 \mathrm{~h}$ ), supporting the likelihood of loss of volatile chemicals during purging in experiment S1. No measurement of $\mathrm{M}_{\mathrm{n}}$ was done for both $\mathrm{S} 1$ and $\mathrm{S} 2$ as the tests were not much successful in terms of conversion.

To overcome the problem of low conversion, purging was done only for the surfactant solution in all subsequent experiments (S3-S7). High conversion obtained in these experiments shows the adverse effect of purging on conversion. Furthermore, a successful ATRP polymerization under a very limited air (oxygen) can be a convenient and economical technique in industry to produce a polymer product with desirable properties.

In S3, the amount of AA was doubled. Adding more reducing agent has resulted in the reasonable conversion of $61 \%$ and average molecular weight of $26579 \mathrm{~g} / \mathrm{mol}$ (PDI $=1.5$ ). This is a typical data of a controlled (living) polymerization 
Table 4.3: Experimental results for single-step emulsion AGET ATRP of MMA

\begin{tabular}{|lllll|}
\hline Exp. & t/h & Conv $(\%)$ & Mn $\left(\mathbf{g m o l}^{-\mathbf{1}}\right)$ & PDI \\
\hline S1 & 2.00 & 6.3 & - & - \\
& 5.00 & 6.4 & - & - \\
S2 & 2.20 & 3.2 & - & - \\
& 2.73 & 18.9 & - & - \\
& 3.23 & 22.3 & - & - \\
S3 & 1.00 & 39.0 & - & - \\
& 2.20 & 61.0 & 26579 & 1.50 \\
S4 & 1.00 & 37.4 & - & - \\
& 2.00 & 66.0 & - & - \\
S5 & 1.50 & 30.7 & 16576 & 1.92 \\
& 3.40 & 64.3 & 20520 & 2.44 \\
S6 & 0.18 & 18.2 & 5849 & 9.70 \\
& 5.50 & 92.2 & 32863 & 3.91 \\
S7 & 1.50 & 35.3 & 10636 & 1.82 \\
& 2.50 & 39.3 & 12371 & 1.91 \\
& 5.50 & 64.8 & 21609 & 2.12 \\
\hline
\end{tabular}

Experiment S4 is very similar to S3, except the impeller was run at a low speed of $150 \mathrm{rpm}$. A conversion of $66 \%$ was obtained after $2 \mathrm{~h}$ of initiation of polymerization, but no $\mathrm{M}_{\mathrm{n}}$ measurement was done.

On improving the monomer conversion in experiments S3 and S4, the system however started to exhibit aggregation and coagulation aspects, which became very severe at higher conversion to the point where some samples could not be collected because of reactor clogging as shown in Figure 4.10. A.

Noting that latex at low conversion was visually free of aggregation, the appearance of aggregation at higher conversion was probably due to poor stabilization of larger polymer particles by the surfactant. Even after the reduction of the impeller speed in experiment $\mathrm{S} 4$, no improvement in the latex stability was obtained. Therefore, the effect of shear induced coagulation is neglected in this type of polymerization system under the given conditions.

Eslami and Zhu, (2006) reported a thermal effect on the latex stabilization, thus the reactor temperature was lowered from $70^{\circ} \mathrm{C}(\mathrm{S} 1-\mathrm{S} 4)$ to $50^{\circ} \mathrm{C}$ (S5-S7). In experiment S5 along with the lowering of temperature, catalyst complex $\left(\mathrm{CuBr}_{2} / \mathrm{dNbpy}\right)$ and reducing agent $(\mathrm{AA})$ were also 
lowered, where clearly improved latex stabilization was observed. In experiment S6, a careful adjustment (slight increase) of the molar ratio of reducing agent was done which resulted in even higher conversion along with no coagulation/aggregation. Though the latex stabilization and conversion were very good in S6, the results of PDI (3.91/5.5h) and average molecular weight $(32863 / 5.5 \mathrm{~h})$ were found to be considerably higher, indicating a poorly controlled system (see Figure 4.3.), which is also clear from the poor fitting of data points in the first order kinetic plot in Figure 4.2. In experiment S7, molar ratios of catalyst complex and reducing agent were kept higher. Though the conversion became lower than in S6, PDI (2.12/5.5h) and $\mathrm{M}_{\mathrm{n}}(21609 \mathrm{~g} / \mathrm{mol})$ values also became lower indicating improved control in the system which is also evidenced by the first order kinetic plot in Figure 4.2.

After trouble shooting for the latex stability and conversion issues by lowering the temperature and by careful adjustment of reactant ratio, the control of the polymerization still remained a concern. For instance, GPC measurements of selected samples in Table 4.3 showed relatively high molecular weight distribution (MWD/PDI) indicating poorly controlled polymerization. Further, relatively high conversion in a short time (S3-S7) and relatively high number average molecular weight from the early stage of polymerization indicated the loss of control over polymerization and low initiation efficiency. Even for experiment S7, which is the best system in terms of overall control (latex stability, conversion, $\mathrm{M}_{\mathrm{n}}$ and PDI), GPC traces (Figure 4.4.) did not shift significantly towards the high molecular weight region with increasing conversion and PDI $(2.12 / 5.5 \mathrm{~h})$ is still relatively high from the perspective of controlled/living polymerization. Increasing PDI (Figure 4.3.) with conversion also contradicts the feature of CRP.

In a previous study (Min and Matyjaszewski, 2009) the single-step emulsion ATRP polymerization was found to suffer from problems of low initiation efficiency and poorly controlled polymerization. Such polymerization deficient aspects are very likely due to the partitioning of catalyst complex in the monomer droplets and the accompanying difficult transfer of the complex towards polymerizing particles. Both micellar and droplet nucleation were assumed to occur inside the same system, where large particles generated from droplet nucleation is likely the main cause of coagulation/aggregation at higher conversion. 


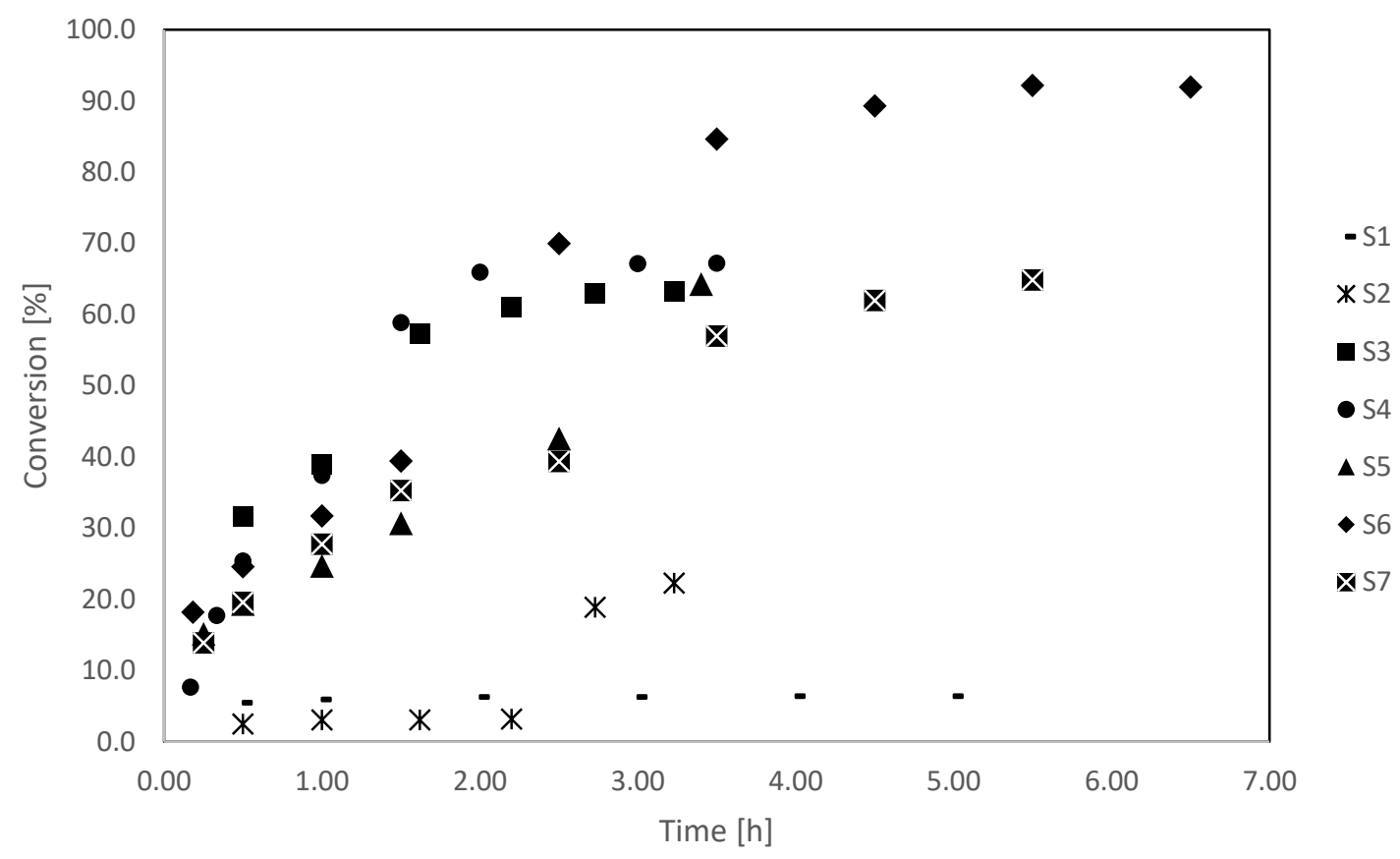

Figure 4.1: MMA conversion versus time in single-step emulsion polymerizations

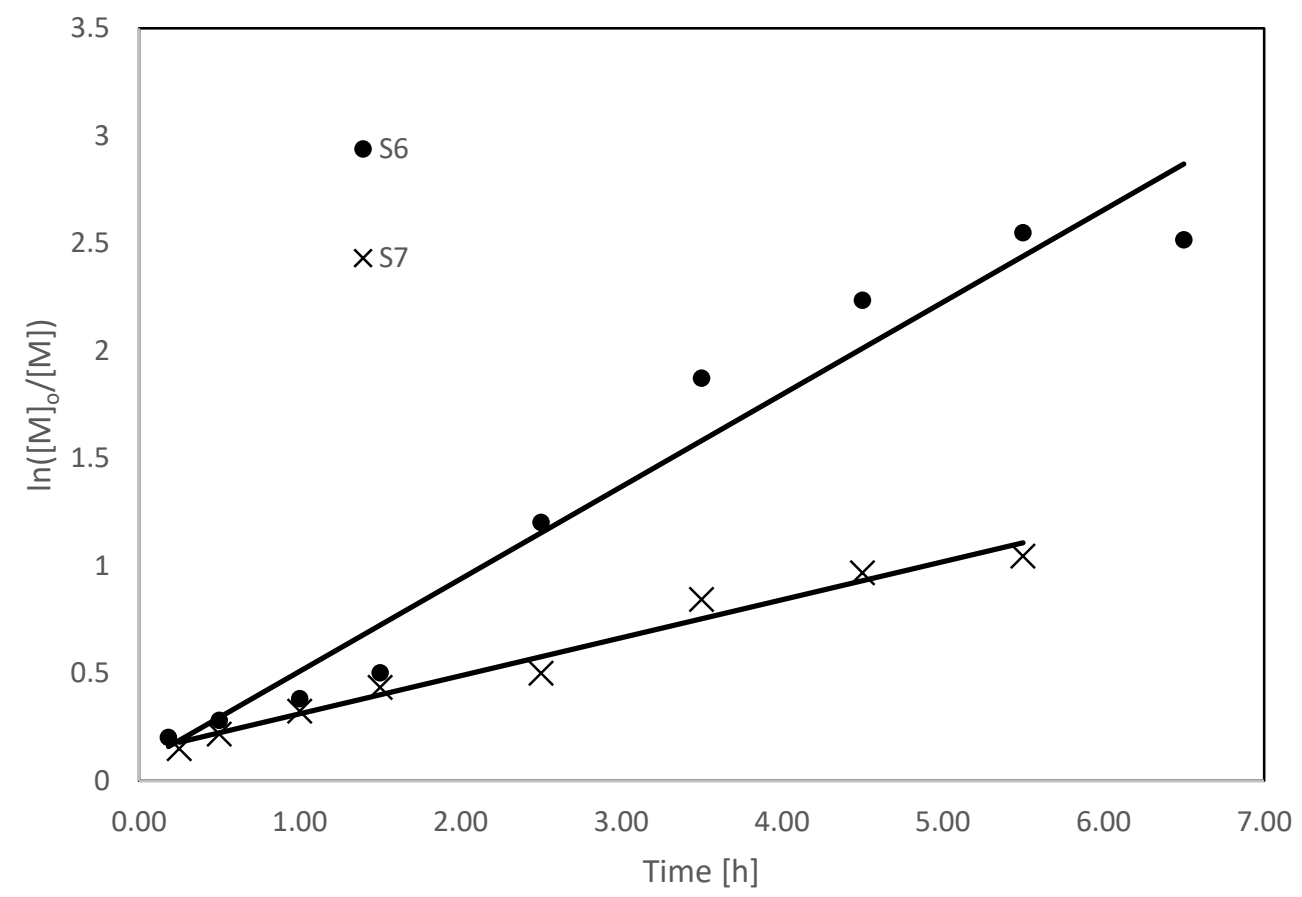

Figure 4.2: Variations of $\ln \left([\mathrm{M}]_{\mathrm{o}} /[\mathrm{M}]\right)$ with reaction time in experiments S6 and S7. 


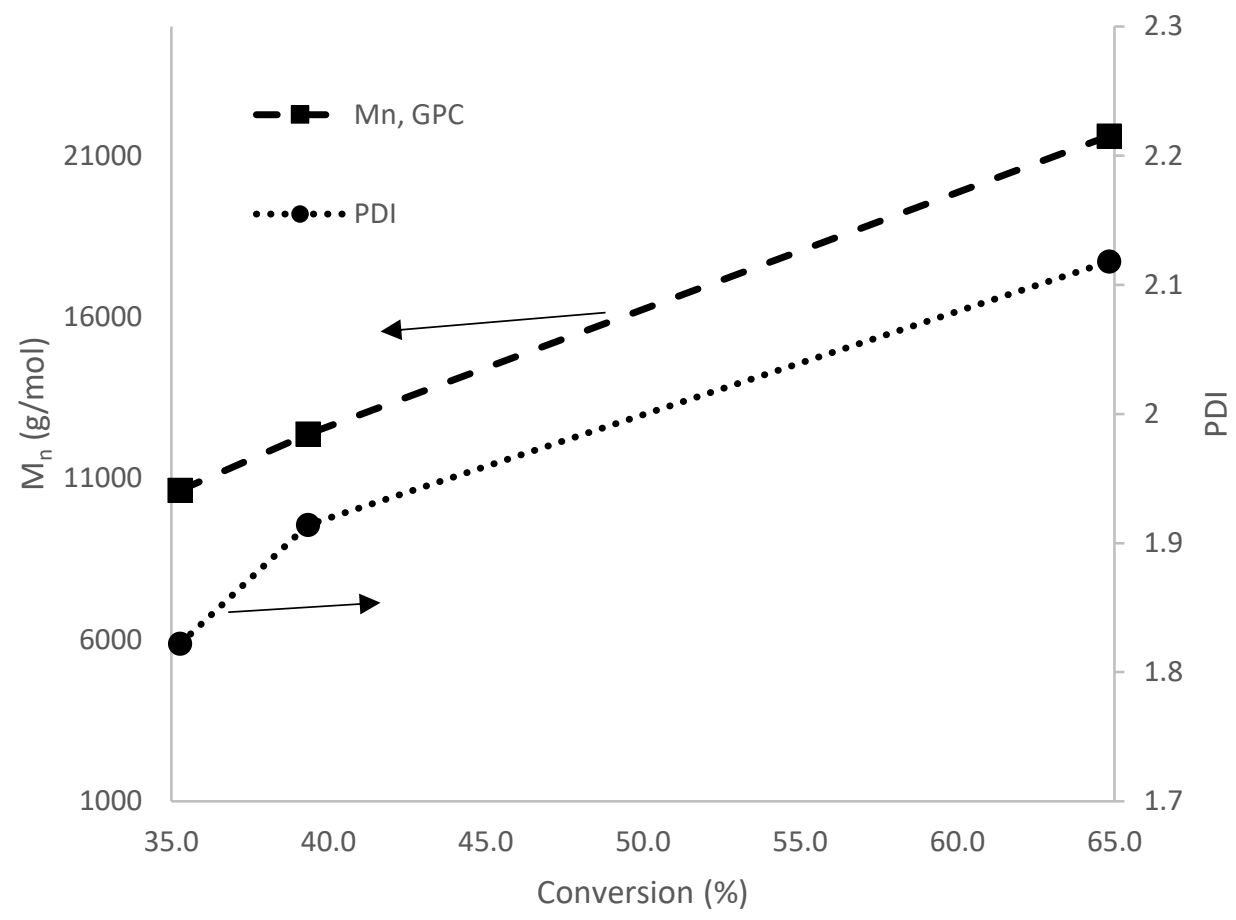

Figure 4.3: Variations of experimental number-average molar mass and polydispersity index (PDI) versus MMA conversions in expt. S7.

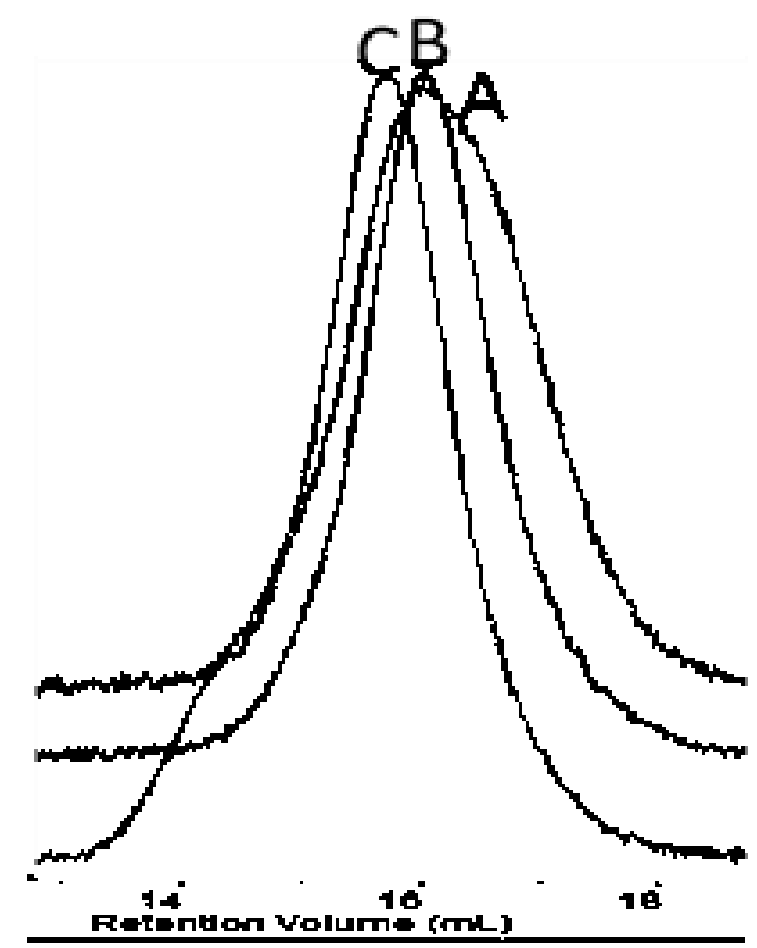

Figure 4.4: A, B, C: GPC traces of PMMA from expt. S7 for $35.5 \%, 39.3 \%$ and $64.8 \%$ conversions, respectively. 


\subsubsection{Two-Step Emulsion Procedure}

A series of PMMA latexes were synthesized via two-step polymerization technique. The runs and the detailed ratio of reagents are listed in Tables 4.4 and 4.5. In a typical polymerization (Run T3), $\mathrm{CuBr}_{2}(0.0838 \mathrm{~g})$ and dNbpy (0.3065 g) were dissolved in MMA I (14.04 g) to form a solution with the catalyst complex. Then initiator (EBiB, $0.4984 \mathrm{~g}$ ) was dissolved in the solution of catalyst complex. The solution of Brij $98(15 \mathrm{~g})$ was prepared in $330 \mathrm{~mL}$ distilled water. The solution of ascorbic acid $(0.06 \mathrm{~g})$ was prepared in $20 \mathrm{~mL}$ water. The organic MMA I solution containing the catalyst complex and initiator was poured slowly into the aqueous solution of Brij 98 at $50^{\circ} \mathrm{C}$ while stirring to form an optically translucent and transparent microemulsion. The microemulsion was transferred to the reactor, then the stirrer was turned on and the mixture was purged with nitrogen gas continuously for $3 \mathrm{~min}$. Then the solution of ascorbic acid (AA I, $10 \mathrm{~mL}$ ) was injected into the reactor at a temperature of $50^{\circ} \mathrm{C}$ to activate the catalyst and start the polymerization. 12 min after initiation, a second portion of methyl methacrylate (MMA II, $42.12 \mathrm{~g}$ ) was added to the ongoing microemulsion polymerization to form an emulsion polymerization, this step was immediately followed by the injection of a second portion of ascorbic acid solution (AA II, $10 \mathrm{~mL}$ ). Polymerization was carried out under an inert blanket of nitrogen gas. Samples were withdrawn at the selected time to measure conversion gravimetrically and to determine molecular weight and molecular weight distribution by GPC. Each aliquot after withdrawal was shaken with air trapped inside the vial and then cooled to stop the polymerization.

\subsubsection{Results and Discussion of Two-Step Emulsion Procedure:}

Because of the poor performance of the single-step emulsion ATRP process as explained above, a two-step emulsion ATRP experimental procedure was attempted. Few past studies have proven the success of the two-step emulsion polymerization technique (Min et al., 2009). Therefore, to get a better control of the polymerization, especially for low PDI/MWD, it was decided to run the two-step emulsion polymerization. Three experimental tests were performed under different experimental conditions as shown in Tables 4.4 and 4.5.

In Table 4.6, the data shows that PDI in all three experiments T1-T3, were fairly close. However, aggregation/coagulation (Figure: 4.10. B) was a serious issue in experiment T1. 
Table 4.4: Recipe for the two-step emulsion AGET ATRP of MMA

\begin{tabular}{|l|l|l|l|l|l|l|l|l|}
\hline Expt. & $\begin{array}{l}\text { MMA I } \\
\text { (g) }\end{array}$ & $\begin{array}{l}\text { MMA } \\
\text { II (g) }\end{array}$ & $\begin{array}{l}\text { EBiB } \\
\text { (g) }\end{array}$ & $\begin{array}{l}\text { CuBr2 } \\
\text { (g) }\end{array}$ & $\begin{array}{l}\text { dNbpy } \\
\text { (g) }\end{array}$ & AA (g) & $\begin{array}{l}\text { Brij 98 } \\
\text { (g) }\end{array}$ & $\begin{array}{l}\text { Water } \\
\text { (g) }\end{array}$ \\
\hline T1 & 4.2120 & 42.1200 & 0.2437 & 0.0197 & 0.0725 & 0.0138 & 9.4000 & 598.2000 \\
\hline T2 & 14.0400 & 42.1200 & 0.4984 & 0.0838 & 0.3065 & 0.0600 & 15.0000 & 348.9500 \\
\hline T3 & 14.0400 & 42.1200 & 0.4984 & 0.0838 & 0.3065 & 0.0600 & 15.0000 & 348.9500 \\
\hline
\end{tabular}

Note: Amount of AA was divided into two equal halves. Second half of AA (in $10 \mathrm{~mL}$ water) was added immediately after the addition of MMA (II).

Table 4.5: Experimental conditions for the two-step emulsion AGET ATRP of MMA

\begin{tabular}{|l|c|c|c|c|}
\hline Expt. & $\begin{array}{l}\text { Temp } \\
\left({ }^{\circ} \mathrm{C}\right)\end{array}$ & $\begin{array}{l}\text { Molar ratio: EBiB: Brij98: MMA(I) } \\
\text { + MMA(II): dNbpy: AA: CuBr2 }\end{array}$ & $\begin{array}{l}\text { Working } \\
\text { volume of the } \\
\text { reactor (mL) }\end{array}$ & $\begin{array}{l}\text { Wt \% of Brij 98 } \\
\text { versus total MMA }\end{array}$ \\
\hline T1 & 70 & $1: 6.5:(34+340): 0.14: 0.06: 0.07$ & 650 & 20 \\
\hline T2 & 60 & $1: 5.1:(55+165): 0.29: 0.13: 0.15$ & 410 & 26.7 \\
\hline T3 & 50 & $1: 5.1:(55+165): 0.29: 0.13: 0.15$ & 410 & 26.7 \\
\hline
\end{tabular}

Note: For all runs, polymerization was carried out under 20-psig pressure of nitrogen gas, stirring rate was $250 \mathrm{rpm}$, and MMA II was added after $12 \mathrm{~min}$ of initiation. Nitrogen purging was done only for microemulsion for $3 \mathrm{~min}$.

High temperature and small amount of surfactant were suspected for poor stabilization of the polymer particles resulting into the serious aggregation/coagulation. Experiment T2 was run at low temperature $\left(60^{\circ} \mathrm{C}\right)$ and with higher loading of the reactants. However, the problems of aggregation/coagulation still persisted in T2. No aggregation till higher conversion $(54 \% / 3 \mathrm{~h})$ in experiment $\mathrm{T} 2$ as compared to that in $\mathrm{T} 1(23 \% / 1.5 \mathrm{~h})$ is believed to be the result of more surfactant loading and low temperature where both factors added up to give more latex stability. Experiment T3 is very similar to T2, except the polymerization was run at even lower temperature $\left(50^{\circ} \mathrm{C}\right)$. Rate of polymerization in $\mathrm{T} 3(35 \% / 5 \mathrm{~h})$ was lower than in $\mathrm{T} 2$. No coagulation in $\mathrm{T} 3(35 \%)$ despite having higher conversion than in $\mathrm{T} 1(22.6 \%)$ is again believed to be the result of low reactor temperature and high surfactant loading. 
Control of polymerization, in terms of PDI $\left(\mathrm{M}_{\mathrm{w}} / \mathrm{M}_{\mathrm{n}}\right)$ and number average molecular weight are much better in two-step emulsion than in single-step emulsion.

Table 4.6: Experimental results for two-step emulsion AGET ATRP of MMA

\begin{tabular}{|ccccc|}
\hline Exp. & t/h & Conv (\%) & Mn (g/mol-1) & PDI \\
\hline T1 & 1 & 15.2 & 63751 & 1.66 \\
& 1.5 & 22.6 & 88123 & 1.64 \\
T2 & 0.5 & 13.0 & 3026 & 1.51 \\
& 1.5 & 34.3 & 9658 & 1.27 \\
& 3 & 53.7 & 16811 & 1.25 \\
T3 & 1 & 12.8 & 2360 & 1.33 \\
& 3 & 24.7 & 3744 & 1.27 \\
& 5 & 34.8 & 4967 & 1.17 \\
\hline
\end{tabular}

In experiment $\mathrm{T} 1$, high values of average molecular weight and relatively broad PDI of 1.66 and 1.64 at $15.2 \%$ and $22.6 \%$ conversion, respectively, indicate low initiation efficiency and a less controlled system. This may be the result of high temperature and low surfactant loading, where stabilization of polymer particles by surfactant became poor and control of polymerization was lost. For experiments T2 and T3, narrow PDI (1.27 and 1.17) and low value of average molecular weights $(9658 \mathrm{~g} / \mathrm{mol}$ and $4967 \mathrm{~g} / \mathrm{mol})$ at the same conversions $(34.3 \%$ and $34.8 \%$ ), indicate well-controlled polymerizations.

First order kinetic plots $\left(\ln \left([\mathrm{M}]_{\mathrm{o}} /[\mathrm{M}]\right)\right.$ vs reaction time) shows a linear trend in two-step emulsion (Figure 4.6) than in single-step emulsion (Figure 4.2), clearly indicating more living/controlled features in the two-step emulsion procedure than in single-step emulsion procedure. Unlike in the single-step procedure (Figure 4.3), PDI in the two-step procedure (Figure 4.7 and 4.8) is decreasing with increasing conversion showing typical feature of CRP. Further, the increase of molecular weight with increasing conversion as indicated by a clear shift in GPC traces (Figure 4.9) confirms that the two-step procedure preserves the living/controlled feature more than the single-step procedure. Despite having obtained well controlled polymerization, latex stability at higher conversion is more challenging in two-step emulsions than in single-step emulsions. This can be caused by a large number of oligomeric chains not growing rapidly to be large enough to effectively stabilize polymeric particles (Oh, 2008). With increasing conversion, escape of $\mathrm{Cu}^{\mathrm{II}}$ species in the aqueous phase can also 
contribute to suppress the solubility of the non-ionic surfactant (Brij 98) in water and prevents it from stabilizing polymer particles (Wei et al., 2015).

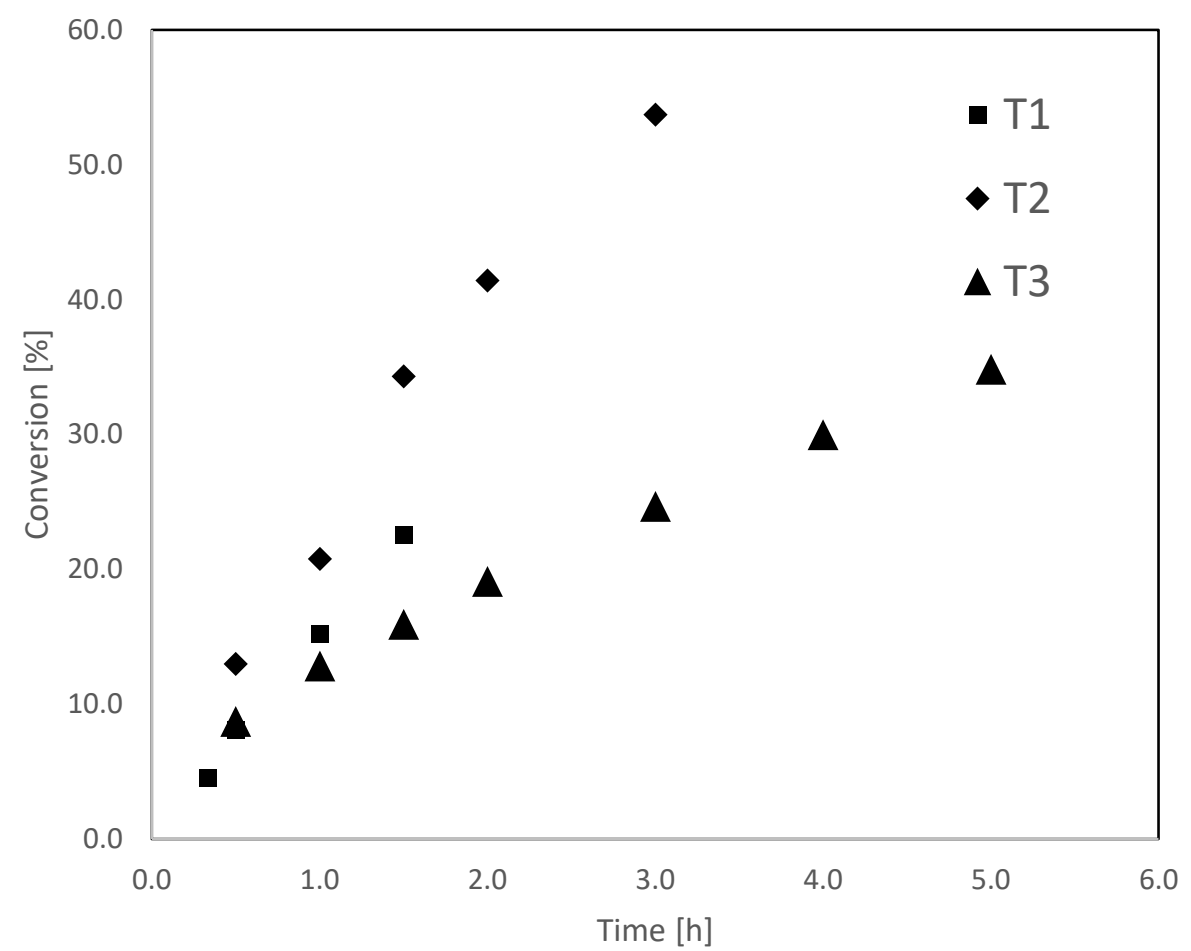

Figure 4.5: MMA conversion versus time in two-step emulsion polymerizations.

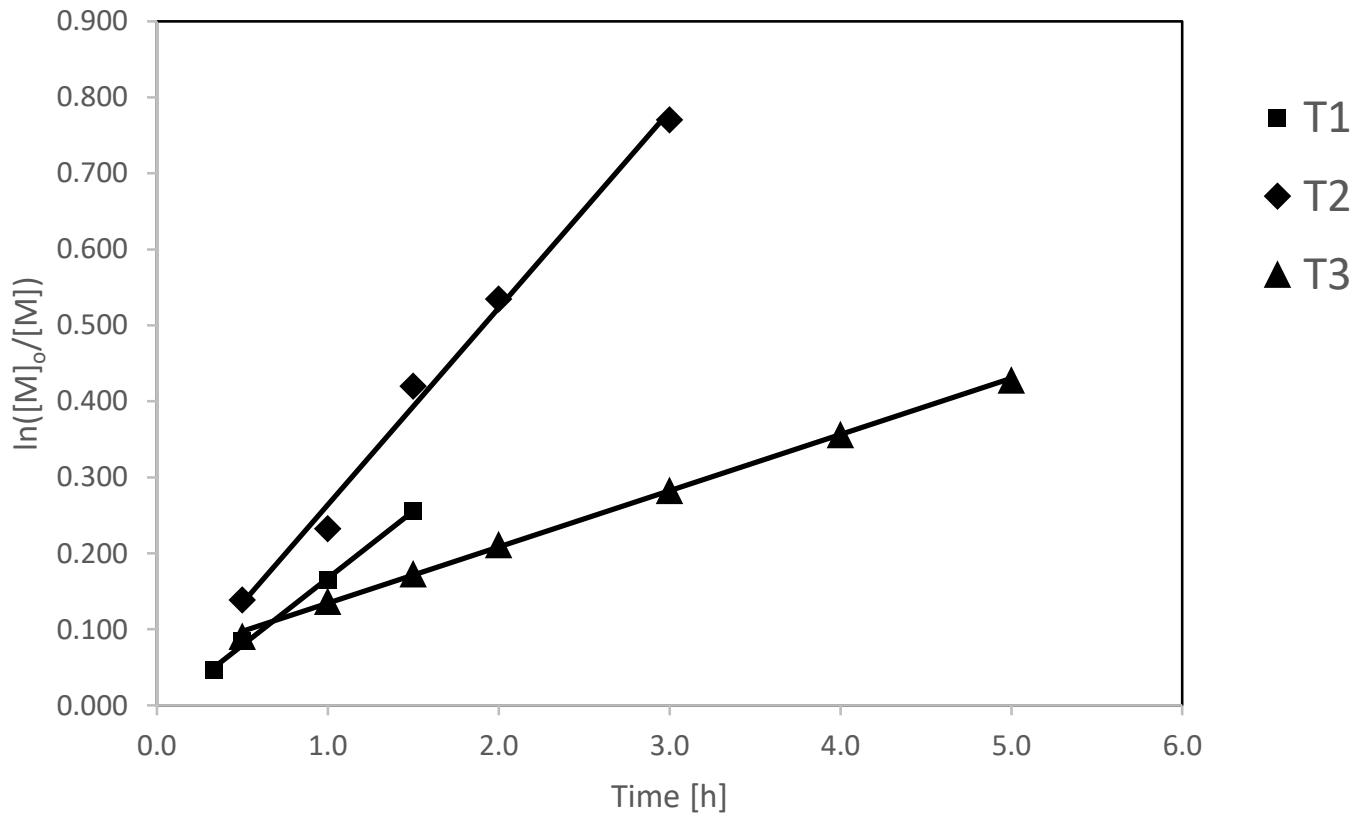

Figure 4.6: Variations of $\ln \left([\mathrm{M}]_{\mathrm{o}} /[\mathrm{M}]\right)$ with reaction time in two-step emulsion polymerizations. 


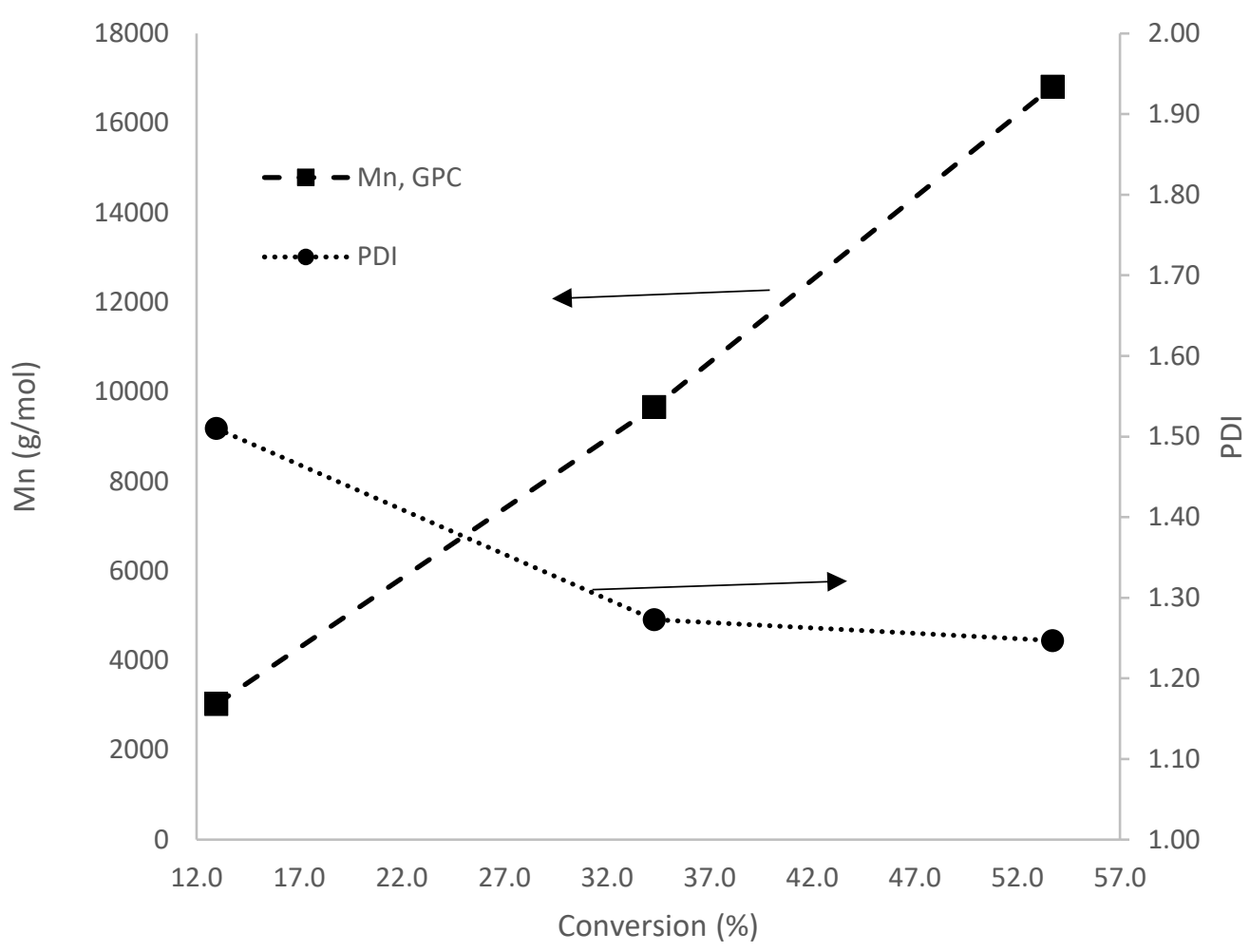

Figure 4.7: Dependence of experimental number-average molar mass $\left(\mathrm{M}_{\mathrm{n}}, \mathrm{g} \mathrm{mol}^{-1}\right)$ and polydispersity index (PDI) on MMA conversions for experiment T2.

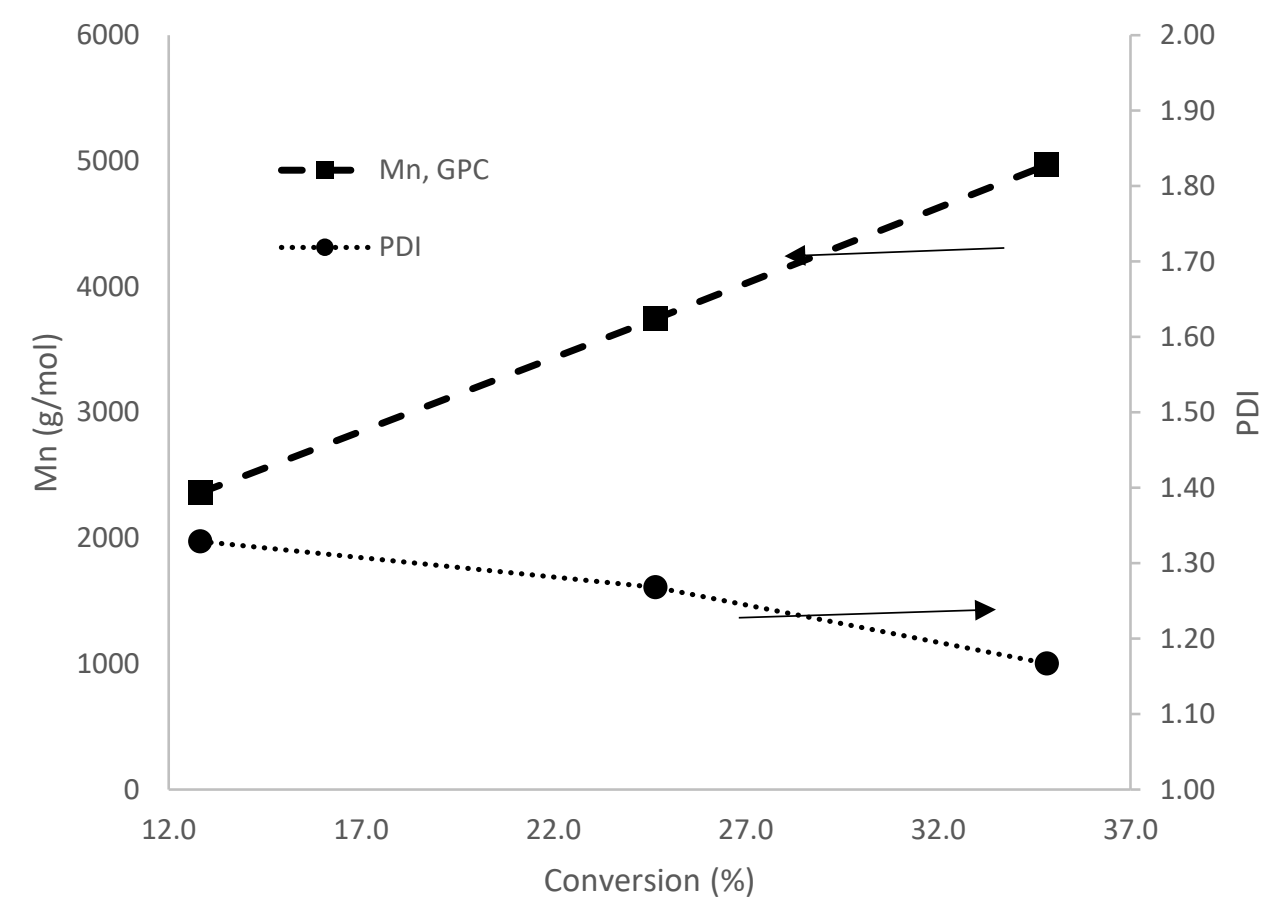

Figure 4.8: Dependence of experimental number-average molar mass $\left(\mathrm{M}_{\mathrm{n}}, \mathrm{g} \mathrm{mol}^{-1}\right)$ and polydispersity index (PDI) on MMA conversions for experiment T3. 


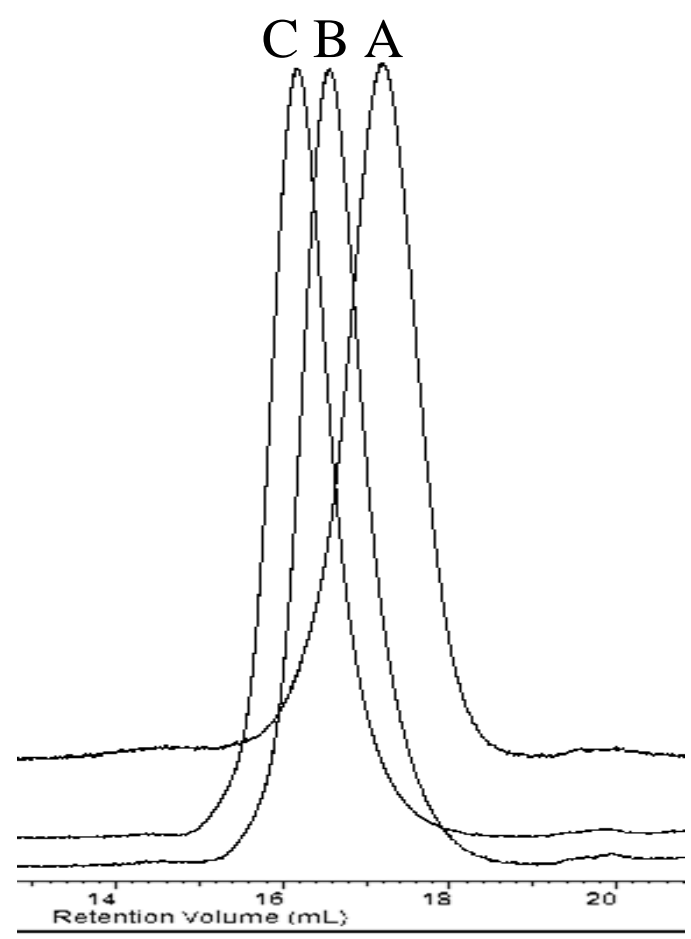

Figure 4.9: A, B, C: GPC traces of PMMA from experiment T3 for 12.8\%, $24.7 \%$ and $34.8 \%$ conversions respectively.

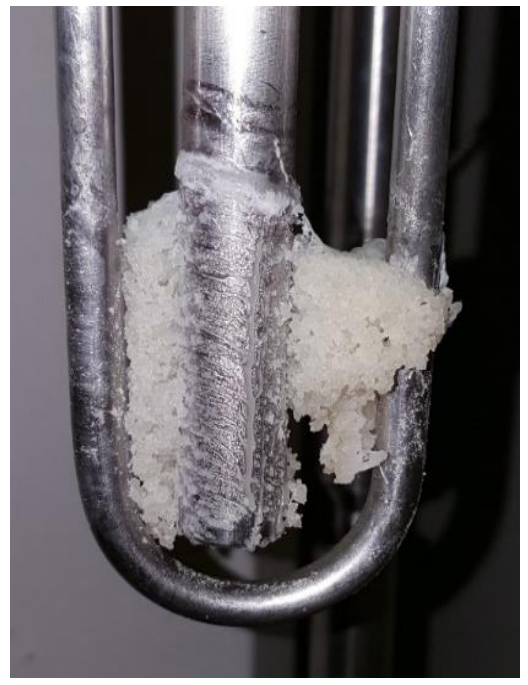

A

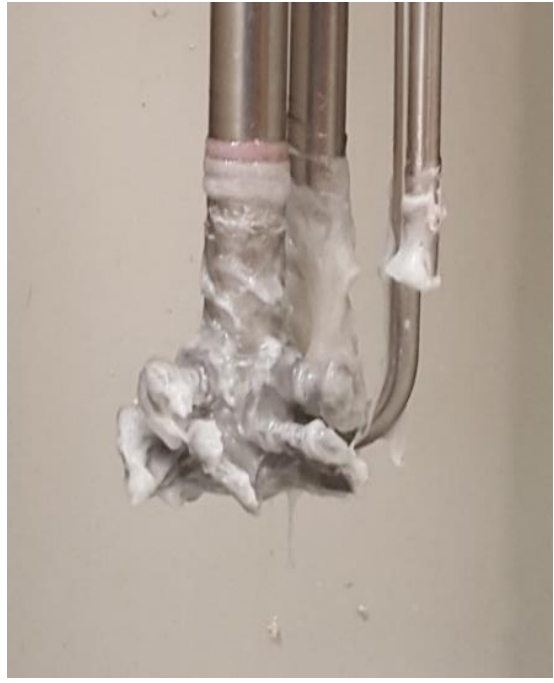

B

Figure 4.10: Aggregation/Coagulation in: A) Single-Step emulsion and B) two-step emulsion. 


\subsection{Concluding Remarks}

Single-step and two-step emulsion AGET ATRP of MMA were performed in a 2-L stirred tank reactor. Air was kept to a minimum level. The aggregation/coagulation phenomenon was found to be a serious issue in both polymerizations. The AGET ATRP polymerizations were much better controlled in the two-step experimental procedure than in the single-step one. In the single-step emulsion with high conversion and good latex stability, the controlled (living) feature was much more compromised. However, the controlled (living) feature of the AGET ATRP polymerization was preserved in the two-step emulsion procedure. Nevertheless, it remains quite challenging to get simultaneously high conversion and good latex stability. Overall, these results show that the two-step emulsion polymerization is more promising than the single-step emulsion polymerization. It can be concluded that with improved latex stability, the two-step emulsion AGET ATRP is certainly a promising polymerization technique to produce well controlled and structured polymers, in case it is thoroughly studied furthermore in academic and industry. 


\section{CHAPTER 5}

\section{EXPERIMENTAL DESIGN AND STATISTICAL ANALYSIS OF TWO-STEP EMUSLION AGET ATRP OF MMA}

\subsection{Introduction}

Most of the experimental studies are usually carried out either in vials or in schlenk flasks, which, while good in laboratory research, cannot be a good representation of the reactors of industrial relevance. The majority of the published papers on polymerization are based on the classical approach of changing one factor at a time to study the effects of factors (independent/input variables) on the process responses (dependent/output variables). Hence, exploring several factors and their effects to investigate the behaviour of a stirred tank reactor in a scientific way by using experimental design and statistical analysis is a topic of interest. From the previous experimental investigation in chapter 4, it was concluded that the two-step emulsion procedure worked much better than the single-step emulsion one to produce a polymer with controlled/living features, which are assessed in terms of lower PDI and relatively low average molecular weights of the polymer. Consequently, the purpose of this chapter is to employ the two-step emulsion method as the experimental procedure to develop an experimental design and statistical analysis to investigate the behaviour of the AGET ATRP of MMA in a 2-L stirred tank reactor in batch mode.

\subsection{Experimental procedure}

This section presents the experimental procedure for two-step emulsion procedure. The materials, reactor setup, reactor operation and polymer characterization methods are discussed in Chapter 3.

The experimental procedure for the two step emulsion system throughout the experimental design is similar to that in Section 4.2.3, except for nitrogen purging and ascorbic acid injection. Here, nitrogen purging was done 10 times, which means that the process of filling the reactor, containing microemulsion, with nitrogen gas to 20 psi and then releasing it fully was repeated consecutively for 10 times to make sure that no air is left. The solution of ascorbic acid was 
prepared in $30 \mathrm{~mL}$ water. Later $15 \mathrm{~mL}$ of ascorbic acid solution was used in two parts. The first part of ascorbic acid (AA I) was $10 \mathrm{~mL}$ and the second part (AA II) was $5 \mathrm{~mL}$ in all experiments.

\subsection{Fractional Factorial Experimental Design}

Since the purpose of this investigation is more than just screening, thus the two level and resolution 5 fractional factorial design $\left(2 \mathrm{v}^{5-1}\right)$ with 5 center points was chosen to develop a set of experiments to investigate thoroughly the effects of the process input variables: temperature, catalyst complex $\left(\mathrm{CuBr}_{2} / \mathrm{dNbpy}\right)$, initiator $(\mathrm{EBiB})$, surfactant (Brij 98) and reducing agent (ascorbic acid (AA)), as well as the dual interactions of each couple of these independent variables on conversion, molecular weight $\left(\mathrm{M}_{\mathrm{n}}\right)$ and polydispersity index (PDI) of the PMMA product.

The Design Expert version 9.0.6 has been used in this study to develop an experimental design and consequently develop an input-output model based on the independent variables values and the related response variables results.

\subsubsection{Tests Based on Experimental Design}

Referring to Table 5.1, the variables $x_{1}, x_{2}, x_{3}, x_{4}$ and $x_{5}$ denote the temperature, surfactant (Brij 98), reducing agent (ascorbic acid), initiator (EBiB) and catalyst complex $\left(\mathrm{CuBr}_{2} / \mathrm{dNbpy}\right)$, respectively. These variables are coded at three levels $+1,0$ and -1 : to represent the highest, average and lowest numerical values as 5.1.

Table 5.1: Experimental range and coded level of the independent variables employed in $2 \mathrm{v}^{5-1}$ fractional factorial design.

\begin{tabular}{|c|c|c|c|c|}
\hline \multicolumn{2}{|c|}{ Independent variable } & \multicolumn{3}{|c|}{ Level and range of independent variable } \\
\hline Actual Variable & Coded variable & -1 & 0 & 1 \\
\hline Temp $\left({ }^{\circ} \mathrm{C}\right)$ & $x_{1}$ or(A) & 50 & 55 & 60 \\
\hline $\begin{array}{l}{ }^{*} \mathrm{CuBr}_{2} / \mathrm{dNbpy} \\
(\mathrm{g} / \mathrm{g})\end{array}$ & $x_{2}$ or(B) & $0.0838 / 0.3065$ & $0.0922 / 0.3372$ & $0.1006 / 0.3678$ \\
\hline Brij $98(\mathrm{~g})$ & $x_{3}$ or $(\mathrm{C})$ & 15.0000 & 16.5000 & 18.0000 \\
\hline $\mathrm{EBiB}(\mathrm{g})$ & $x_{4}$ or(D) & 0.3987 & 0.4486 & 0.4984 \\
\hline Ascorbic acid (g) & $x_{5}$ or $(\mathrm{E})$ & 0.0600 & 0.0660 & 0.0720 \\
\hline
\end{tabular}

*The molar ratio of catalyst $\left(\mathrm{CuBr}_{2}\right)$ to ligand (dNbpy) is kept constant at 1:2 in every run. 
Based on the data in table 5.1, a set of 21 experiments was generated by the Design Expert software version 9.0.6 from Stat-Ease, based on the experimental design $2{ }_{\mathrm{v}}{ }^{5-1}$. Following the experimental procedure described in Section 5.2, the 21 experiments were carried out using the values of the reactants and temperature shown in Table 5.2.

Table 5.2: Five factor two-level fractional factorial experimental design with resolution $5\left(2 \mathrm{v}^{5-1}\right)$.

\begin{tabular}{|c|c|c|c|c|c|}
\hline \multicolumn{6}{|c|}{ Independent Coded Variables } \\
\hline Run & $\operatorname{Temp}\left({ }^{\circ} \mathrm{C}\right)$ & Brij 98 (g) & Ascorbic acid (g) & EBiB (g) & $\begin{array}{l}\text { Catalyst complex }(\mathrm{g} / \mathrm{g}) \\
\mathrm{CuBr}_{2} / \mathrm{dNbpy}\end{array}$ \\
\hline 1 & $60(1)$ & $15.0000(-1)$ & $0.0600(-1)$ & $0.3987(-1)$ & $0.0838 / 0.3065(-1)$ \\
\hline 2 & $60(1)$ & $18.0000(1)$ & $0.0600(-1)$ & $0.4984(1)$ & $0.0838 / 0.3065(-1)$ \\
\hline 3 & $50(-1)$ & $18.0000(1)$ & $0.0600(-1)$ & $0.3987(-1)$ & $0.0838 / 0.3065(-1)$ \\
\hline 4 & $60(1)$ & $15.0000(-1)$ & $0.0720(1)$ & $0.3987(-1)$ & $0.1006 / 0.3678(1)$ \\
\hline 5 & $55(0)$ & $16.5000(0)$ & $0.0660(0)$ & $0.4486(0)$ & $0.0922 / 0.3372(0)$ \\
\hline 6 & $60(1)$ & $18.0000(1)$ & $0.0720(1)$ & $0.3987(-1)$ & $0.0838 / 0.3065(-1)$ \\
\hline 7 & $60(1)$ & $15.0000(-1)$ & $0.0600(-1)$ & $0.4984(1)$ & $0.1006 / 0.3678(1)$ \\
\hline 8 & $60(1)$ & $15.0000(-1)$ & $0.0720(1)$ & $0.4984(1)$ & $0.0838 / 0.3065(-1)$ \\
\hline 9 & $50(-1)$ & $15.0000(-1)$ & $0.0600(-1)$ & $0.4984(1)$ & $0.0838 / 0.3065(-1)$ \\
\hline 10 & $60(1)$ & $18.0000(1)$ & $0.0600(-1)$ & $0.3987(-1)$ & $0.1006 / 0.3678(1)$ \\
\hline 11 & $60(1)$ & $18.0000(1)$ & $0.0720(1)$ & $0.4984(1)$ & $0.1006 / 0.3678$ \\
\hline 12 & $50(-1)$ & $18.0000(1)$ & $0.0720(1)$ & $0.4984(1)$ & $0.0838 / 0.3065(-1)$ \\
\hline 13 & $50(-1)$ & $18.0000(1)$ & $0.0600(-1)$ & $0.4984(1)$ & $0.1006 / 0.3678$ \\
\hline 14 & $50(-1)$ & $18.0000(1)$ & $0.0720(1)$ & $0.3987(-1)$ & $0.1006 / 0.3678$ \\
\hline 15 & $55(0)$ & $16.5000(0)$ & $0.0660(0)$ & $0.4486(0)$ & $0.0922 / 0.3372(0)$ \\
\hline 16 & $50(-1)$ & $15.0000(-1)$ & $0.0720(1)$ & $0.3987(-1)$ & $0.0838 / 0.3065(-1)$ \\
\hline 17 & $50(-1)$ & $15.0000(-1)$ & $0.0600(-1)$ & $0.3987(-1)$ & $0.1006 / 0.3678$ \\
\hline 18 & $55(0)$ & $16.5000(0)$ & $0.0660(0)$ & $0.4486(0)$ & $0.0922 / 0.3372(0)$ \\
\hline 19 & $55(0)$ & $16.5000(0)$ & $0.0660(0)$ & $0.4486(0)$ & $0.0922 / 0.3372(0)$ \\
\hline 20 & $55(0)$ & $16.5000(0)$ & $0.0660(0)$ & $0.4486(0)$ & $0.0922 / 0.3372(0)$ \\
\hline 21 & $50(-1)$ & $15.0000(-1)$ & $0.0720(1)$ & $0.4984(1)$ & $0.1006 / 0.3678(1)$ \\
\hline
\end{tabular}

Following condition is kept constant for all runs (1-21):

\begin{tabular}{|l|l|}
\hline $\mathrm{N}_{2}$ purging & Only for microemulsion (10 times, as explained in Section 5.2) \\
\hline Motor Speed & $250 \mathrm{rpm}$ \\
\hline Pressure & $20 \mathrm{psi}$ \\
\hline MMA I $(\mathrm{g})$ & 14.0400 \\
\hline MMA II $(\mathrm{g})$ & 42.1200 \\
\hline MMA total $(\mathrm{g})$ & 56.1600 \\
\hline Water $(\mathrm{g})$ & 348.9500 \\
\hline
\end{tabular}


All experimental runs were conducted in the stirred tank reactor under a nitrogen blanket. The samples were collected at designated times and were subsequently analyzed to determine conversion, molecular weight and polydispersity index. The reaction time is a very important parameter of the ATRP polymerization process. The FFD procedure is run for the 5 independent variables listed in Table 5.1 and statistical analysis is carried out using the experimental values of samples collected at a given fixed time. Hence, the FFD program will generate a time invariant input-output model. Therefore, samples were collected exactly after $2 \mathrm{~h}$ reaction time for each experiment and they were analyzed to determine the MMA conversion, $\mathrm{M}_{\mathrm{n}}$ and PDI of the polymer samples, thus collected. Time is not treated as an independent variable.

Even though the conversion of MMA is relatively low in the AGET ATRP process, the results of the $\mathrm{M}_{\mathrm{n}}$ and PDI are very encouraging. They reflect that this process is effectively a controlled/living polymerization. The reaction condition in the 2-L batch reactor did not allow us to carry out the reaction for longer time.

Table 5.3: Experimental results of the measured responses

\begin{tabular}{|cccc|}
\hline \multicolumn{4}{|c|}{ Process Responses } \\
\hline Run & Conv, \% & Mn (g/mol) & PDI \\
\hline 1 & 35.4 & 29981 & 1.210 \\
2 & 48.2 & 19012 & 1.358 \\
3 & 22.6 & 13314 & 1.203 \\
4 & 35.3 & 23277 & 1.249 \\
5 & 29.3 & 13894 & 1.312 \\
6 & 49.6 & 21623 & 2.476 \\
7 & 28.5 & 11093 & 1.619 \\
8 & 33.3 & 10524 & 1.657 \\
9 & 19.1 & 9665 & 1.267 \\
10 & 32.9 & 17292 & 1.399 \\
11 & 43.0 & 11872 & 1.925 \\
12 & 25.7 & 10252 & 1.419 \\
13 & 18.7 & 13038 & 1.166 \\
14 & 22.3 & 12906 & 1.265 \\
15 & 30.2 & 12667 & 1.308 \\
16 & 22.0 & 11455 & 1.307 \\
17 & 19.7 & 10183 & 1.244 \\
18 & 30.4 & 13610 & 1.361 \\
19 & 29.7 & 10263 & 1.312 \\
20 & 26.7 & 12464 & 1.320 \\
21 & 20.3 & 10709 & 1.140 \\
\hline
\end{tabular}




\subsubsection{Model Development}

The experimental results in Table 5.3 show that the conversion varied from $18.7 \%$ (run 13) to $49.6 \%$ (run 6), whereas the number average molecular weights $\left(M_{n}\right)$ varied from $9665 \mathrm{~g} / \mathrm{mol}$ (run 9) to $29981 \mathrm{~g} / \mathrm{mol}$ (run 1). The polydispersity index of the polymer sample varied from 1.140 (run 21) to 2.476 (run 6). Once a design of experiments has been constructed and the related experimental testing have been carried out, it is customary to use the input and output data to construct a model based on the regression principles. Considering that higher order interactions between the factors ( 3 factors interaction or more) are neglected and the quadratic effects cannot be estimated with high confidence by the proposed FFD experimental design, the experimental data were fitted to the following second-order equation (e.g., 5.1).

$y_{k}=b_{o}+\sum_{i=1}^{n} b_{i} x_{i}+\sum_{i=1}^{n-1} \sum_{j=2}^{n} b_{i j} x_{i} x_{j}$

where $k$ varies from 1 to $\mathrm{m}$ with $\mathrm{m}$ being the number of process outputs.

$y_{k}$ represents the response variable (process output), $n$ is the number of independent variables, $b_{o}$ is the regression coefficient at the intercept and $b_{i}$ and $b_{i j}$ are the regression coefficients for the linear and interaction of each factor $x_{i}$ respectively. According to the design of experiments, the mathematical model is used for theoretical prediction of response variables.

In terms of coded factors, the following model was established which includes all independent variables and their two factor interactions.

$$
\begin{aligned}
& y_{1}=29.66+8.49 x_{1}-2.20 x_{2}+3.09 x_{3}-0.19 x_{4}+1.65 x_{5}-1.15 x_{1} x_{2}+2.06 x_{1} x_{3}+ \\
& 0.16 x_{1} x_{4}+0.37 x_{1} x_{5}-1.45 x_{2} x_{3}+0.22 x_{2} x_{4}+0.99 x_{2} x_{5}+1.21 x_{3} x_{4}+0.63 x_{3} x_{5}- \\
& 0.68 x_{4} x_{5} \\
& y_{2}=14242.57+3322.00 x_{1}-966.00 x_{2}+151.38 x_{3}-2741.62 x_{4}-685.00 x_{5}- \\
& 1234.75 x_{1} x_{2}-785.87 x_{1} x_{3}-2217.38 x_{1} x_{4}-575.25 x_{1} x_{5}-170.63 x_{2} x_{3}+623.37 x_{2} x_{4}+ \\
& 1579.75 x_{2} x_{5}+1371.50 x_{3} x_{4}-65.38 x_{3} x_{5}-496.38 x_{4} x_{5} \\
& y_{3}=1.41+0.18 x_{1}-0.056 x_{2}+0.095 x_{3}+0.012 x_{4}+0.12 x_{5}-0.008 x_{1} x_{2}+ \\
& 0.083 x_{1} x_{3}+0.016 x_{1} x_{4}+0.092 x_{1} x_{5}-0.032 x_{2} x_{3}+0.074 x_{2} x_{4}-0.10 x_{2} x_{5}- \\
& 0.072 x_{3} x_{4}+0.12 x_{3} x_{5}-0.032 x_{4} x_{5}
\end{aligned}
$$

Where $y_{1}, y_{2}$ and $y_{3}$ are the conversion (\%), the PMMA molecular weight $(\mathrm{g} / \mathrm{mol})$ and the polydispersity index of molecular weight (PDI) respectively; whereas $x_{1}, x_{2}, x_{3}, x_{4}$ and $x_{5}$ are 
independent (input) variables defined in Table 5.1. The model can be used to predict the response variables at other experimental conditions within the ranges specified in Table 5.2.

Referring to Table 5.4, based on the $p$-value of the coefficients, the algorithm has excluded from the model (5.2) - (5.4) the non-significant two factor interactions: $x_{1} x_{4}, x_{1} x_{5}, x_{2} x_{4}$ and $x_{3} x_{5}$ in conversion, $x_{2} x_{3}, x_{4} x_{5}, x_{2} x_{4}$ and $x_{3} x_{5}$ in molecular weight and $x_{1} x_{2}$ and $x_{1} x_{4}$ in PDI. This exclusion resulted into improved residual analysis at the cost of reduced value of $\mathrm{R}^{2}$ and $\mathrm{R}^{2}$ adj.

The predictive quality and reliability of the model are discussed in Section 5.3.3.

Table 5.4: Regression coefficients and probability values of statistical analysis for the prediction of the response variables.

\begin{tabular}{|ccccccc|}
\hline $\begin{array}{c}\text { Regression } \\
\text { Coefficient }\end{array}$ & $\begin{array}{c}\text { Coefficient for } \\
\text { conversion }\end{array}$ & $\boldsymbol{p}$-value & $\begin{array}{c}\text { Coefficient for } \\
\text { polymer MW }\end{array}$ & $\boldsymbol{p}$-value & $\begin{array}{c}\text { Coefficien } \\
\text { t for PDI }\end{array}$ & $\boldsymbol{p}$-value \\
\hline b1 & 8.49 & $<0.0001$ & 3322 & $<0.001$ & 0.18 & $<0.0001$ \\
b2 & -2.2 & 0.0003 & -966 & 0.0239 & -0.056 & 0.0006 \\
b3 & 3.09 & $<0.0001$ & $151.38^{*}$ & 0.6661 & 0.095 & $<0.0001$ \\
b4 & $-0.19^{*}$ & 0.5909 & -2741.63 & $<0.0001$ & $0.012^{*}$ & 0.1950 \\
b5 & 1.65 & 0.0016 & $-685^{*}$ & 0.0810 & 0.12 & $<0.0001$ \\
b12 & -1.15 & 0.0106 & -1234.75 & 0.0079 & $-0.008^{*}$ & 0.2178 \\
b13 & 2.06 & 0.0004 & $-785.88^{*}$ & 0.0520 & 0.083 & $<0.0001$ \\
b14 & $0.16^{*}$ & 0.6860 & -2217.38 & 0.0003 & 0.016 & 0.0452 \\
b15 & $0.37^{*}$ & 0.3723 & $-575.25^{*}$ & 0.1307 & 0.092 & $<0.0001$ \\
b23 & -1.45 & 0.0033 & $-170.62^{*}$ & 0.6580 & -0.032 & 0.0093 \\
b24 & $0.22^{*}$ & 0.5795 & $623.38^{*}$ & 0.1561 & 0.074 & 0.0001 \\
b25 & 0.99 & 0.0209 & 1579.75 & 0.0022 & -0.1 & $<0.0001$ \\
b34 & 1.21 & 0.0083 & $1371.5^{*}$ & 0.0047 & -0.072 & 0.0001 \\
b35 & $0.63 *$ & 0.1026 & $-65.37 *$ & 0.8638 & 0.12 & $<0.0001$ \\
b45 & $-0.68^{*}$ & 0.0822 & $-496.38^{*}$ & 0.2373 & -0.032 & 0.0094 \\
& & & & & & \\
$\mathbf{R}^{2}(\%)$ & 98.78 & & 94.35 & & 97.21 & \\
$\mathbf{R}^{2}$ adj $(\%)$ & 97.29 & & 85.87 & & 92.04 & \\
$\mathbf{R}_{\text {pred }}^{2}(\%)$ & 93.69 & & 56.13 & & 54.81 & \\
Adeq precision $^{2}$ & 30.261 & & 13.802 & & 18.968 & \\
\hline$*$ Insignificant coefficient values based on the corresponding $p$-value $\geq 0.05$ & & \\
\hline
\end{tabular}




\subsubsection{Statistical Data Analysis}

Based on analysis of variance (ANOVA) results and the multi-regression method, the second order Equations (5.2) - (5.4) were found to accurately represent the experimental data. As shown in Figures 5.3 (D), 5.8 (D) and 5.13 (D), there is a good agreement between the experimental data and the model with the coefficients of determination $\left(\mathrm{R}^{2}\right)$ of $98.78,94.35$ and $97.21 \%$ respectively. Table 5.4 shows the value of coefficients of each response variables (conversion \%, molecular weight $\mathrm{g} / \mathrm{mol}$ and PDI) and the $p$-value of each coefficient in the model. The high values of coefficient of determination $\mathrm{R}^{2}$ and $\mathrm{R}_{\text {adj }}^{2}$ indicate that the developed model (Equations 5.2-5.4) predict the experimental data reliably well within the experimental conditions of the experiments.

Table 5.5: ANOVA summary of the model for conversion, molecular weight and PDI.

\begin{tabular}{|c|c|c|c|c|c|c|}
\cline { 2 - 7 } \multicolumn{1}{c|}{} & \multicolumn{2}{c|}{ Conversion } & \multicolumn{2}{c|}{ Molecular weight } & \multicolumn{2}{c|}{ PDI } \\
\hline & $F$-value & $p$-value & $F$-value & $p$-value & $F$-value & $p$-value \\
\hline Model & 75.28 & $<0.0001$ & 23.70 & 0.0002 & 121.37 & $<0.0001$ \\
\hline Curvature & 0.60 & 0.4648 & 10.04 & 0.0157 & 39.22 & 0.0008 \\
\hline Lack of Fit & 0.52 & 0.6911 & 0.73 & 0.5856 & 5.20 & 0.0771 \\
\hline
\end{tabular}

\subsection{Results and Discussion of Model Predictions}

The model predictive capability of each process variable: Conversion, $\mathrm{M}_{\mathrm{n}}$ and PDI is discussed next.

\subsubsection{Conversion}

The monomer is the most important reactant in a polymerization system. The rate of consumption of the monomer is principally affected by the reaction temperature, initiator and catalyst. Nevertheless, the remaining reactants can also impact on the monomer conversion. An analysis of variance (ANOVA) was carried out to determine the significant effects and main interaction effects of factors which causes influence on the conversion. 
For the selection of statistically significant effects half-normal plot is far easier to use and more likely to result in a correctly "picked" model as compared to normal plot and Pareto chart. Referring to Figure 5.1, the vertical line in the half normal probability plot is the reference standard, points away from this line represent larger effect. Filled points represent unselected effects. Triangular points are for the estimation of error. Black points are for positive effects and grey points are for negative effects.

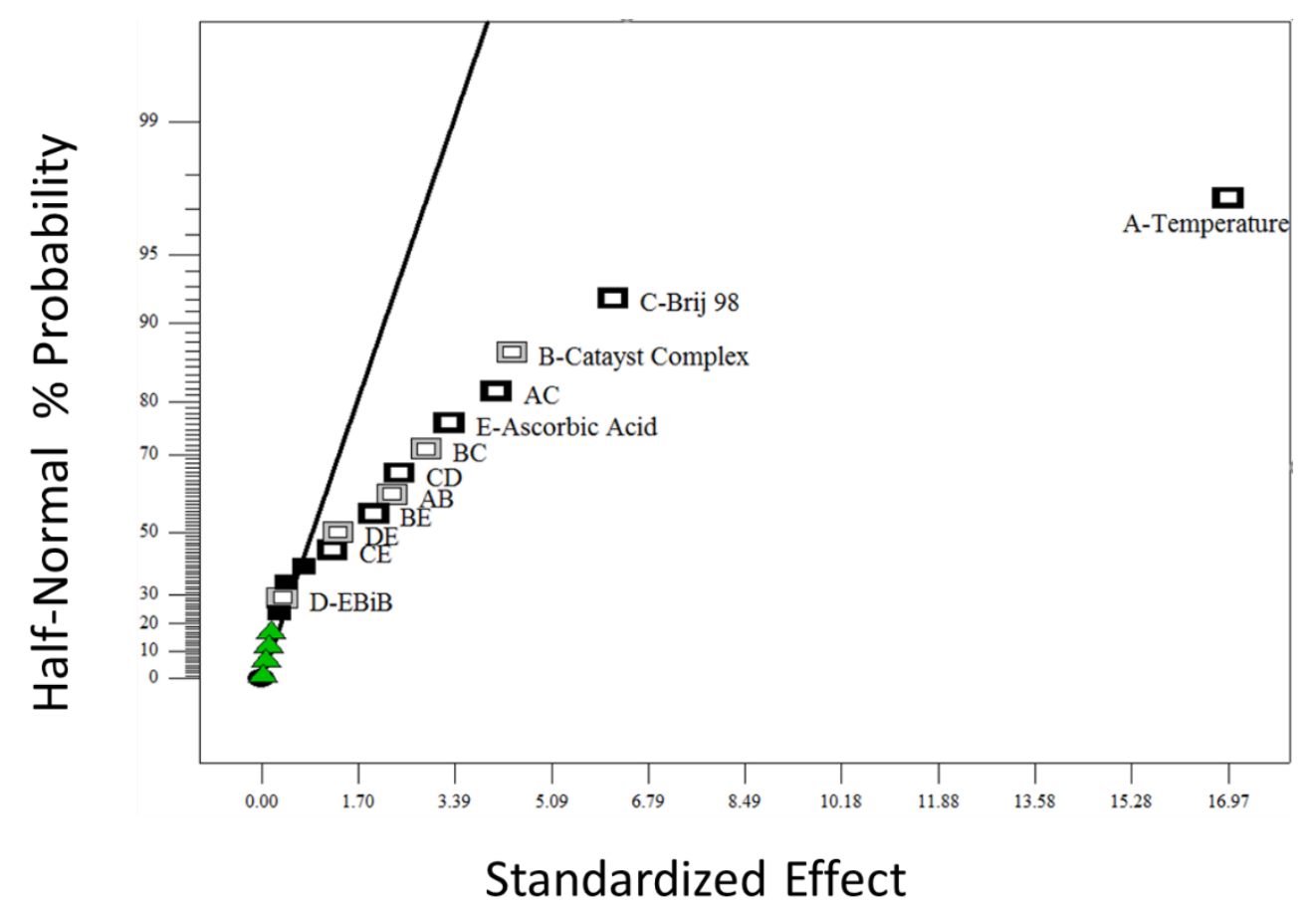

Figure 5.1: Half normal probability plot for the selection of main factors and two factor interaction effects influencing conversion

Referring to Figure 5.2, the Pareto chart shows the absolute values of main input variables and interaction factors after the selection of effects on the half-normal plot. Effects above the Bonferroni Limit are significant. Effects above the t-Value Limit are possibly significant. Effects below the t-Value limit are not likely to be significant. Only filled bars represent not selected effects. In Appendix D, Table D.1 and D.2 show the details of ANOVA results about conversion. 


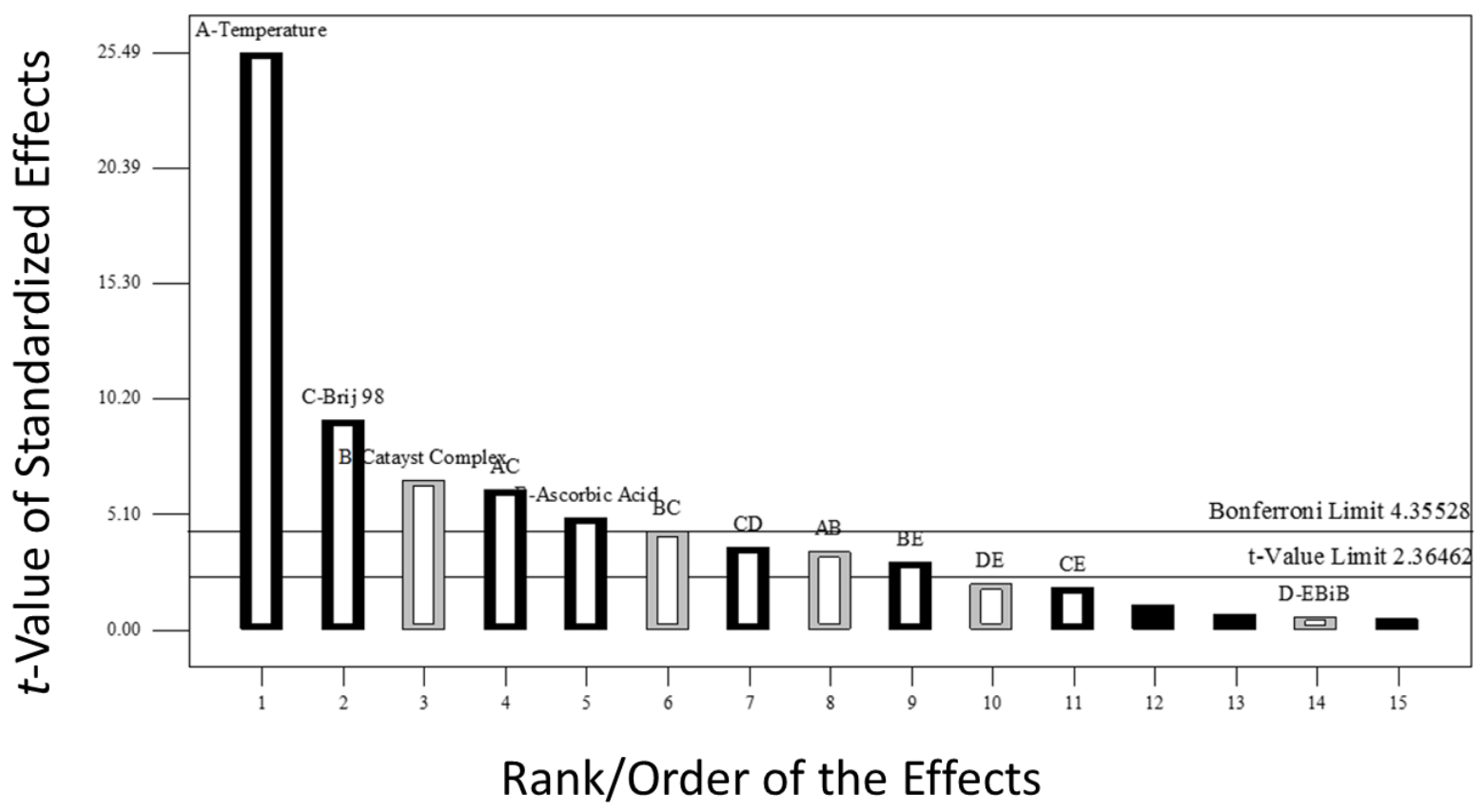

Figure 5.2: Pareto chart with the list of effects according to their influence level on conversion.

The adequate precision value of 30.261 (minimum desired value is 4) in Table 5.4, indicates an adequate signal, hence this model can be used to navigate the design space. Referring to Table 5.4 along with the half-normal probability plot (Figure5.1), effects list (Table 5.6.) and Pareto chart (Figure 5.2) it is found that A, B, C, E, AB, AC, BC, BE, CD are significant terms. Factor D on its own is not significant, however for hierarchical reason, this factor was included for the analysis. The model $F$-value of 75.28 along with insignificant curvature and insignificant lack of fit (Table 5.5) proves the significance and validity of the model.

There are four main diagnostic plots to check the assumptions of ANOVA, and they are: normal probability plot of residuals, residuals versus predicted plot, residual versus run plot and predicted versus actual plot (Figure 5.3.). Studentizing the residuals maps all the different normal distributions to a single standard normal distribution. Externally studentized residuals based on a deletion method are more sensitive than internally studentized residuals to find problems with the analysis. Hence plots of externally studentized residuals are used for the analysis. 
Table 5.6: Effects list showing \% contribution of main factors and two factor interaction effects on conversion.

\begin{tabular}{|c|c|c|c|}
\hline Term & $\begin{array}{c}\text { Stdized } \\
\text { Effect }\end{array}$ & $\begin{array}{c}\text { Sum of } \\
\text { Squares }\end{array}$ & $\begin{array}{c}\text { \% } \\
\text { Contribution }\end{array}$ \\
\hline A-Temperature & 16.97 & 1152.6 & 71.34 \\
B-Catalyst Complex & -4.4 & 77.44 & 4.79 \\
C-Brij 98 & 6.18 & 152.52 & 9.44 \\
D-EBiB & -0.37 & 0.56 & 0.035 \\
E-Ascorbic acid & 3.3 & 43.56 & 2.7 \\
AB & -2.3 & 21.16 & 1.31 \\
AC & 4.13 & 68.06 & 4.21 \\
AD & 0.32 & 0.42 & 0.026 \\
AE & 0.75 & 2.25 & 0.14 \\
BC & -2.9 & 33.64 & 2.08 \\
BD & 0.45 & 0.81 & 0.05 \\
BE & 1.98 & 15.6 & 0.97 \\
CD & 2.42 & 23.52 & 1.46 \\
CE & 1.25 & 6.25 & 0.39 \\
DE & -1.35 & 7.29 & 0.45 \\
Curvature & -0.51 & 1.06 & 0.066 \\
Lack of Fit & & 0 & 0 \\
Pure Error & & 8.93 & 0.55 \\
\hline
\end{tabular}

Viewed from Figure 5.3 (A), all the residuals are close to the straight line; therefore, the normal distribution assumption is satisfied. In Figure 5.3 (B), because all the residual points are scattered randomly all over the graph within the upper and lower bounds instead of accumulating in the other areas, thus the assumption of constant variance/ homoscedasticity is fulfilled. Figure 5.3 (C) shows that all the residual points are spread within upper and lower bounds, showing no pattern, which verifies the assumption of independence. In Figure 5.3 (D), all the points are close to the straight line, showing that the 'predicted vs actual plot' is satisfactory and the model fits well. Hence all diagnostic plots indicate that all the required assumptions of ANOVA are fulfilled. 


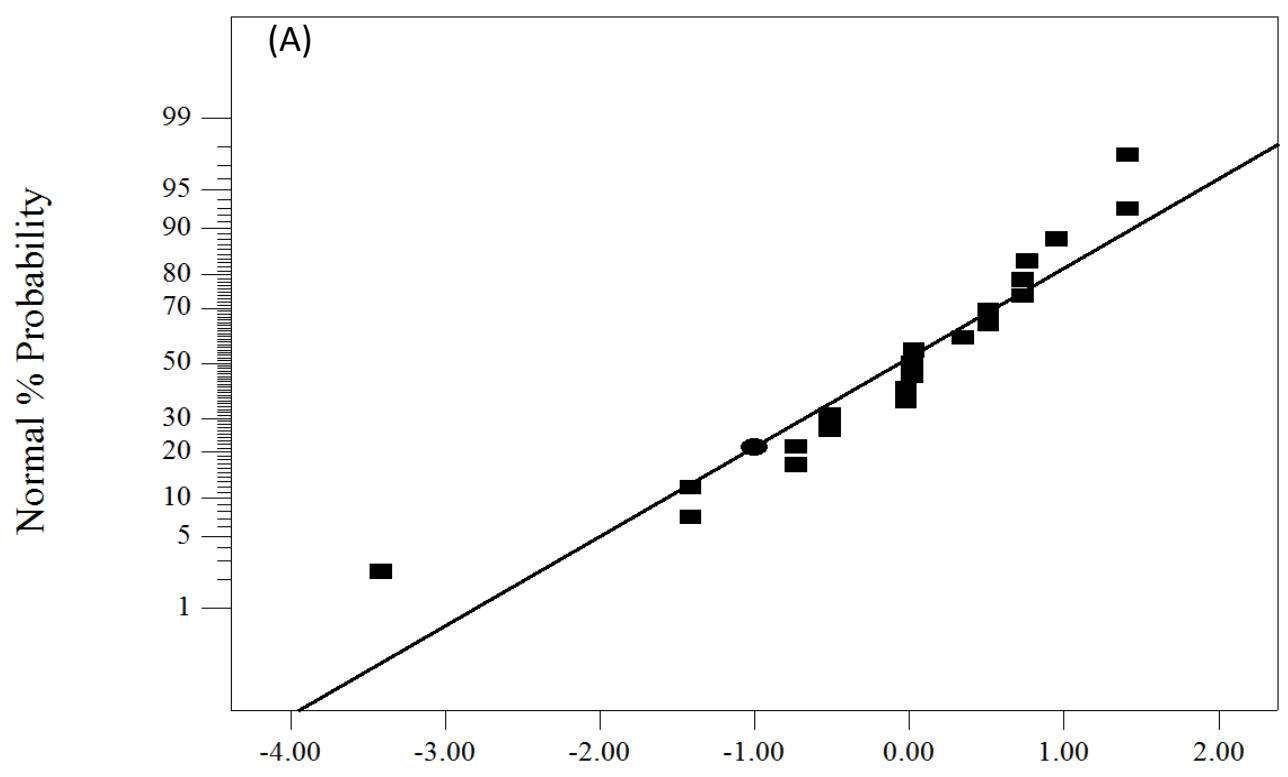

Externally Studentized Residuals

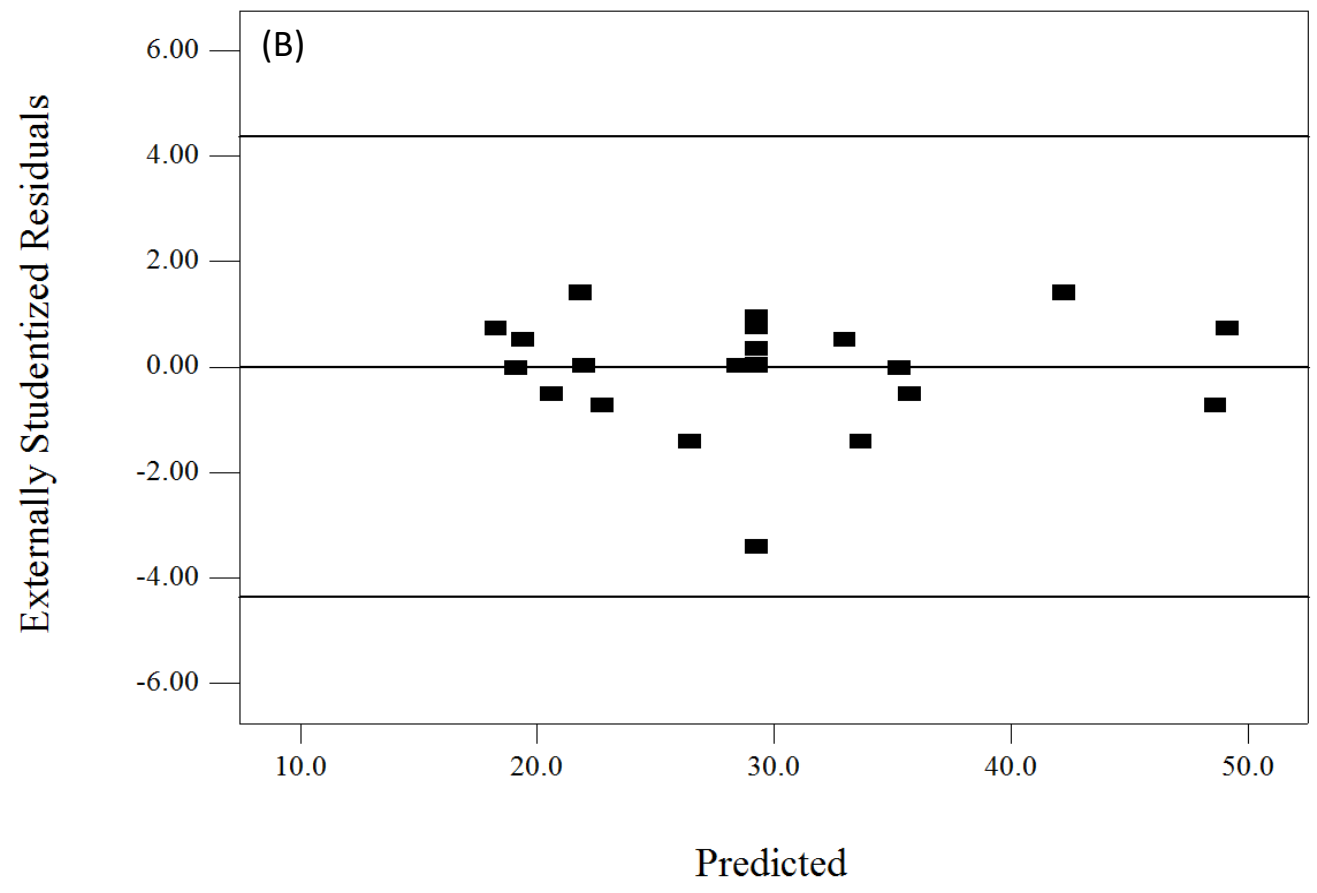




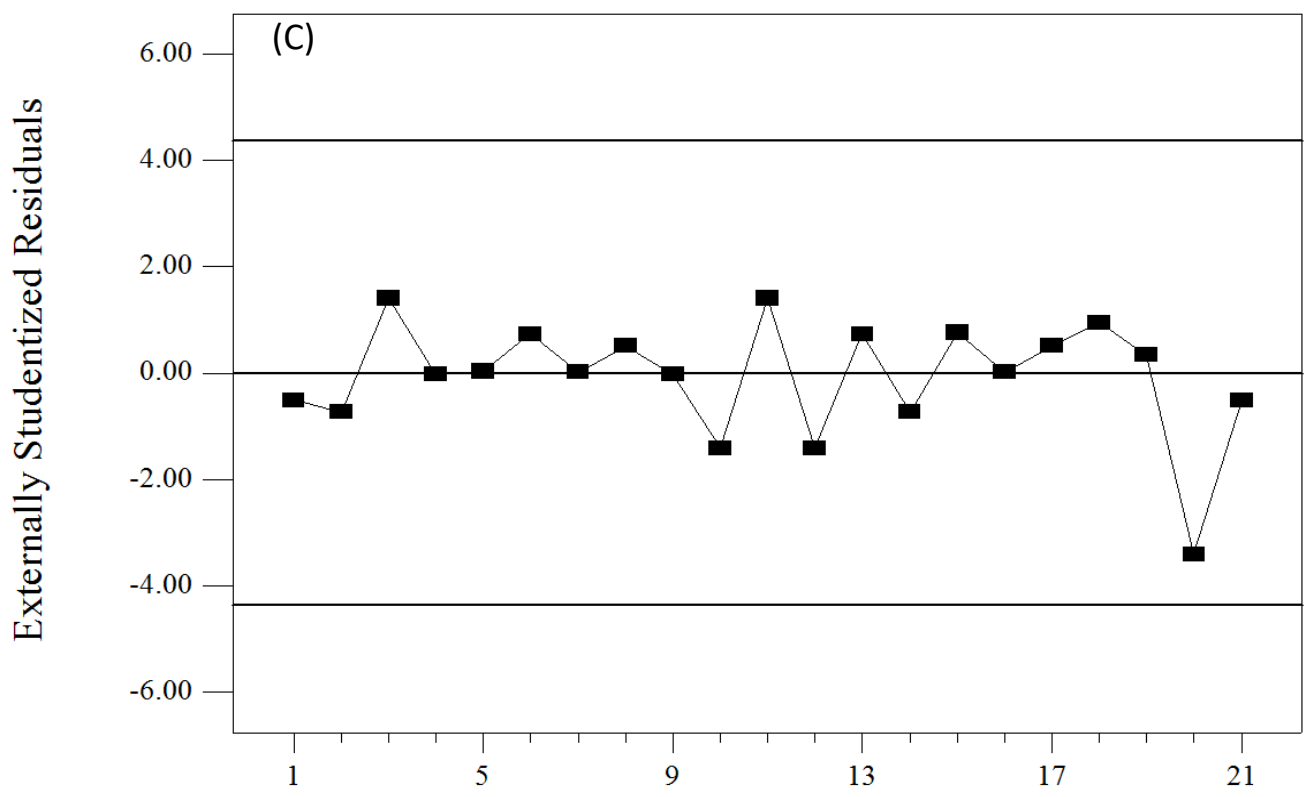

Run Number

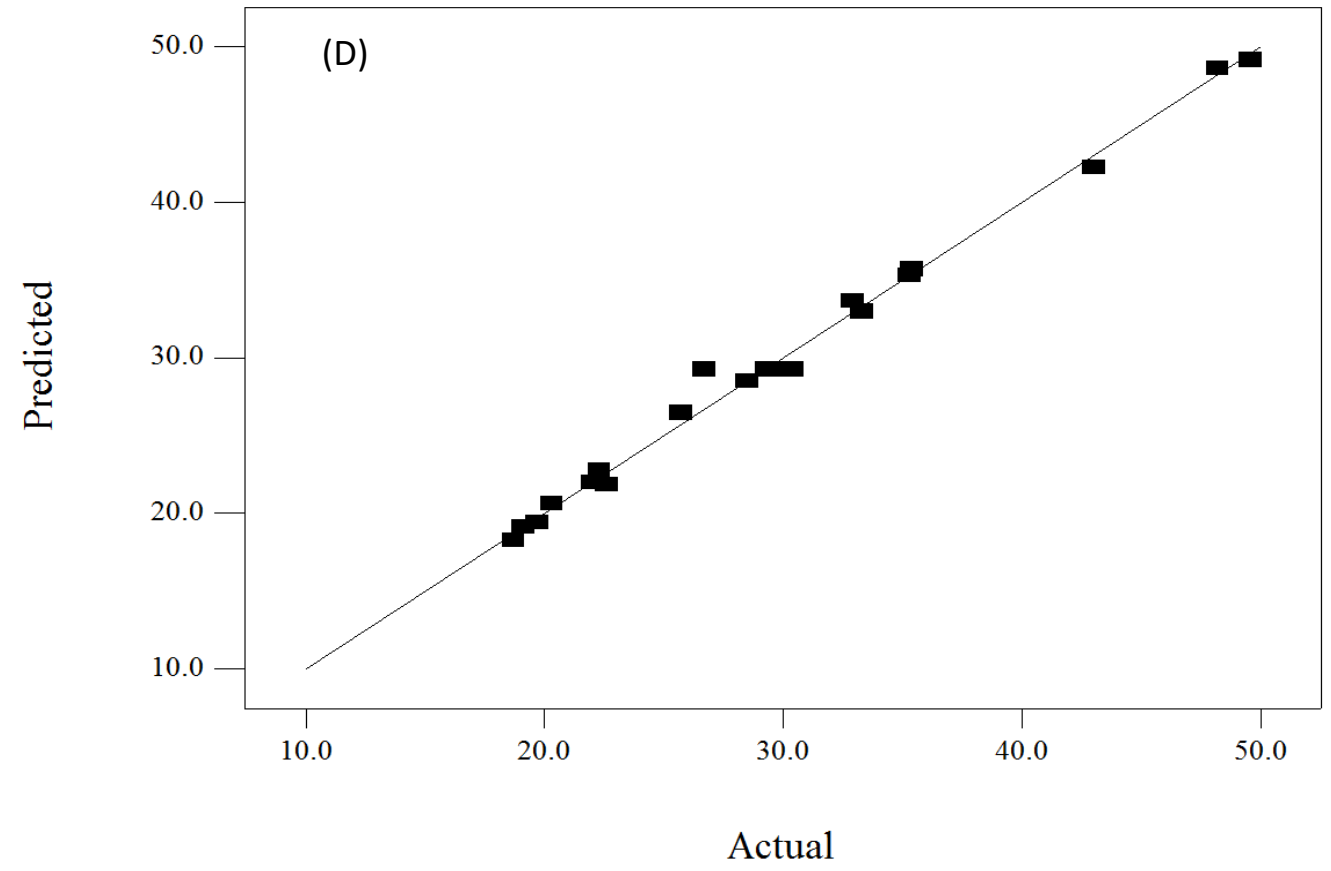

Figure 5.3: Diagnostic plots for conversion: (A) normal probability plot of residuals, (B) residuals vs predicted (C) residuals vs run and (D) predicted vs actual. 


\section{a) Individual Effects of Independent Variables on Conversion}

Compiling the data of all 21 experimental runs (Tables 5.2. and 5.3.), the plots shown in Figures 5.4 were generated to study the individual effects of process parameters. Each plot depicts the variation of conversion with respect to one key factor while the other four factors were kept invariant at their respective central points. According to Table 5.4, the $p$-values of all the independent variables but $\mathrm{EBiB}$ are less than 0.05 . Hence each key factor except initiator (EBiB) can significantly affect the conversion. However, for hierarchical reasons it was included for the analysis as its interaction with surfactant 'Brij 98' (CD) is significant with a $p$-value smaller than 0.05 .

As can be seen from the Pareto chart (Figure 5.2) and effects list (Table 5.6), temperature has highest influence $(71.34 \%)$ on conversion, followed by Brij 98 (9.44\%), catalyst complex $(4.79 \%)$ and ascorbic acid (2.7\%). Effect of EBiB is not significant (0.035\%) for conversion. Only the catalyst complex and $\mathrm{EBiB}$ have a negative impact on conversion, whereas the other independent factors have positive impact.

The increased conversion with increasing temperature can be attributed to the increased $K_{p}$, $\mathrm{K}_{\mathrm{act}}$ and $\mathrm{K}_{\mathrm{ATRP}}$ values (Qu et al., 2013; Seeliger and Matyjaszewski, 2009; Tang et al., 2008).

Decreased conversion with increased catalyst complex can be attributed to the high proportion of deactivator over the activator, which according to Equation 2.1, results in the lower monomer conversions (Matyjaszewski et al., 1997).

By controlling the particle size and number, the surfactant concentration can control the polymerization rate and molecular weight. With increasing surfactant, there will be more micelles for nucleation to occur, which results in increased monomer conversion (Pan et al., 2002).

Increased reducing agent concentration likely keeps deactivator concentration low by converting deactivator into activator, changing the copper(I)/copper(II) ratio and increasing reaction rate as evidenced by Equation 2.1.

The negligible effect of initiator concentration on conversion is more likely to be the result of higher proportion of initiator as compared to the catalyst concentration. It is believed that the activation deactivation step controls the rate of polymerization when number of active sites far outweighs the number of catalyst species in the system. 


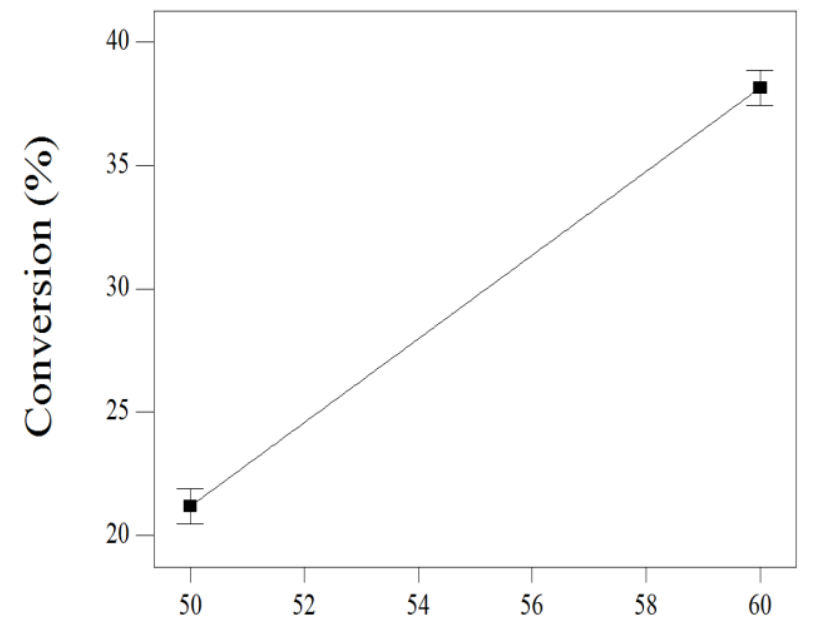

A: Temperature $\left({ }^{\circ} \mathrm{C}\right)$
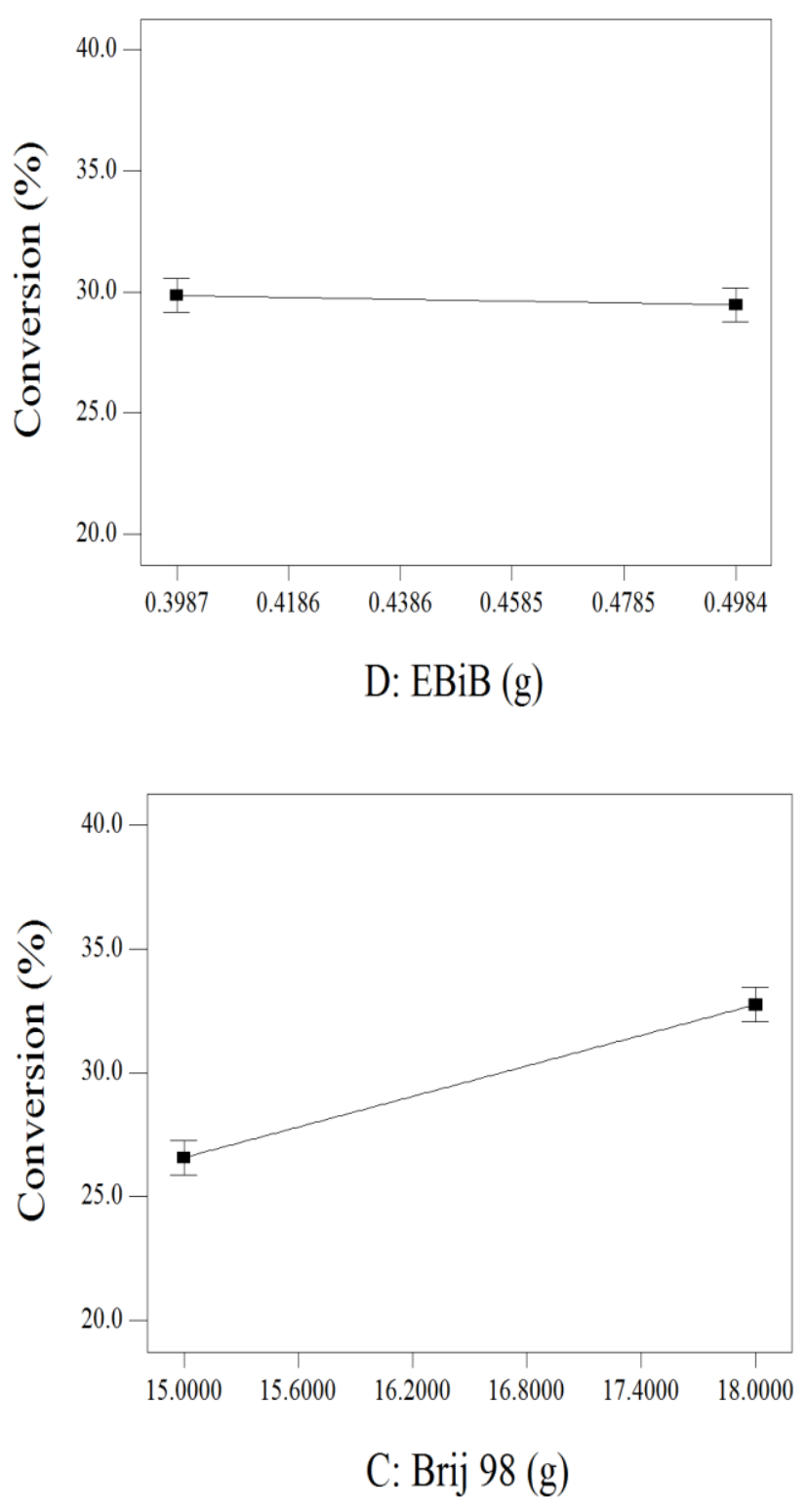
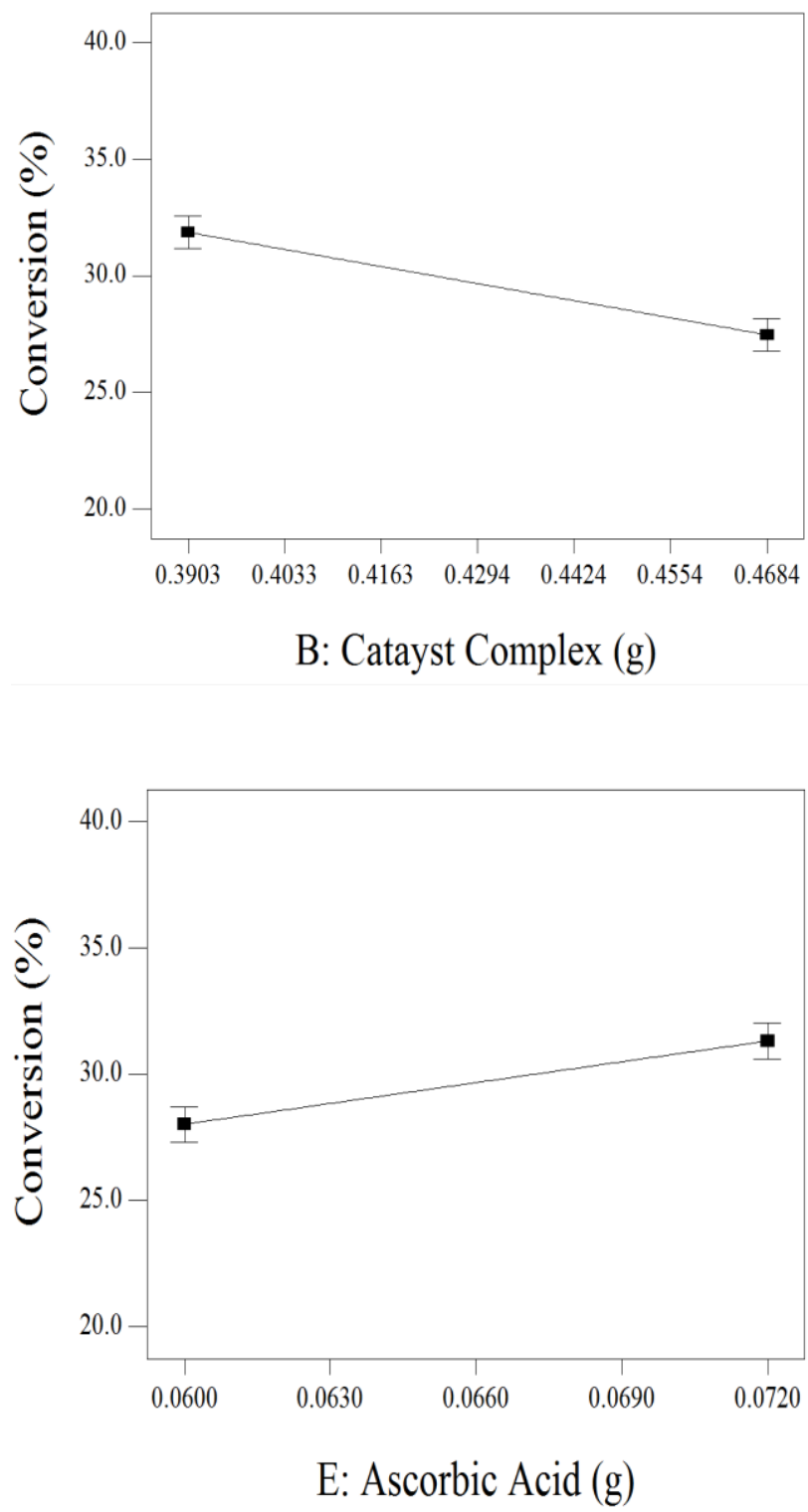

Figure 5.4: Effects of process inputs on the conversion 


\section{b) Effects of Interactions of Independent Variables on Conversion}

The interaction effect plots are shown in Figure. 5.5. The non-parallel lines in this Figure show an interaction between the two factors. The coefficients in Table 5.4, show 7 positive and 3 negative interactions. However, based on the $p$-value (Table 5.4) 4 positive interactions: temperature and surfactant (AC), surfactant and initiator (CD), catalyst complex and reducing agent (BE) as well as surfactant and reducing agent (CE) are found to be significant. Similarly, two negative interactions: temperature and catalyst complex $(\mathrm{AB})$ and catalyst complex and surfactant (BC) are found to be significant. As evidenced from Pareto chart (Figure 5.2) and coefficient $p$-value (Table 5.4) interaction between the temperature and surfactant is the most important positive interaction and the interaction between the catalyst complex and surfactant is the most important negative interaction.

In the Figure 5.5 the points with triangle symbols and square symbols are for the factors (shown in top of each graph) at high levels and low levels respectively. For another factor involved in the interaction (shown at the bottom of each graph), labels on $\mathrm{x}$-axis show the low and high levels. 


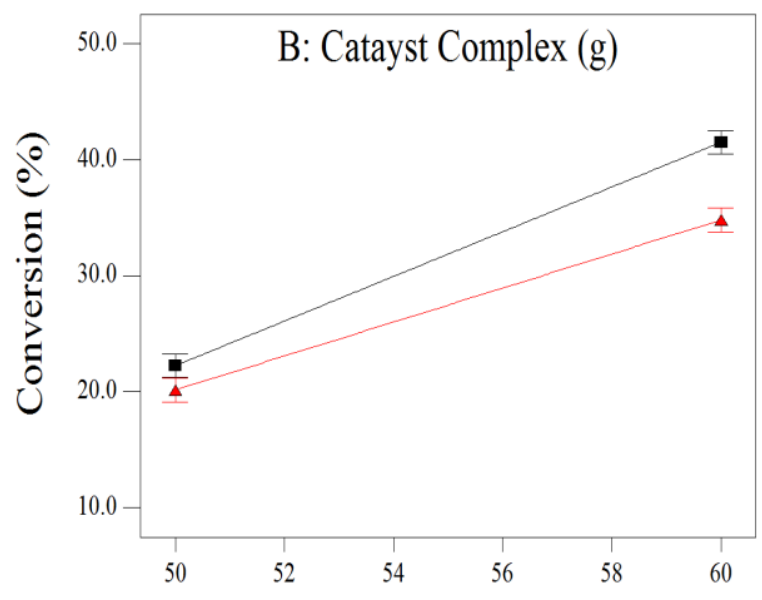

A: Temperature $\left({ }^{\circ} \mathrm{C}\right)$
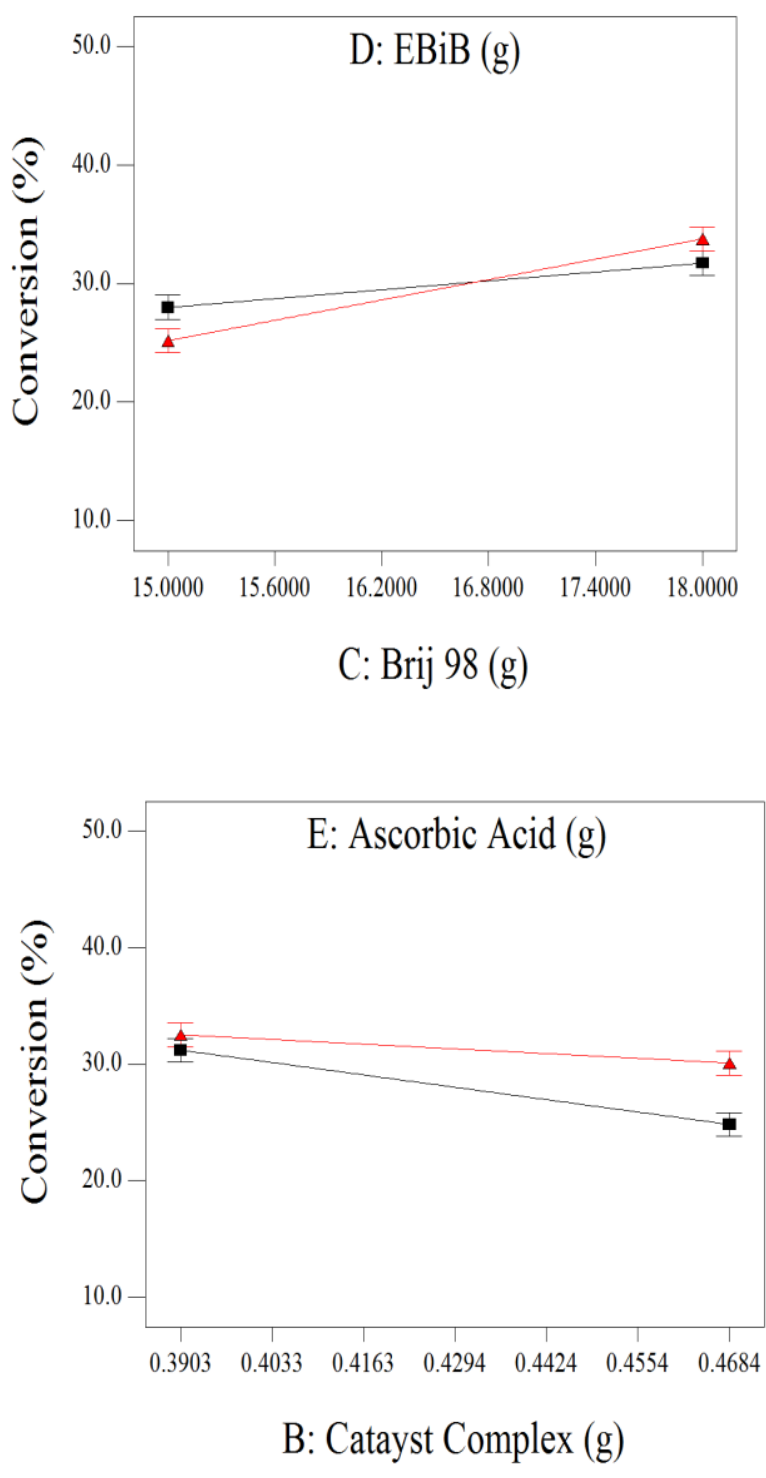

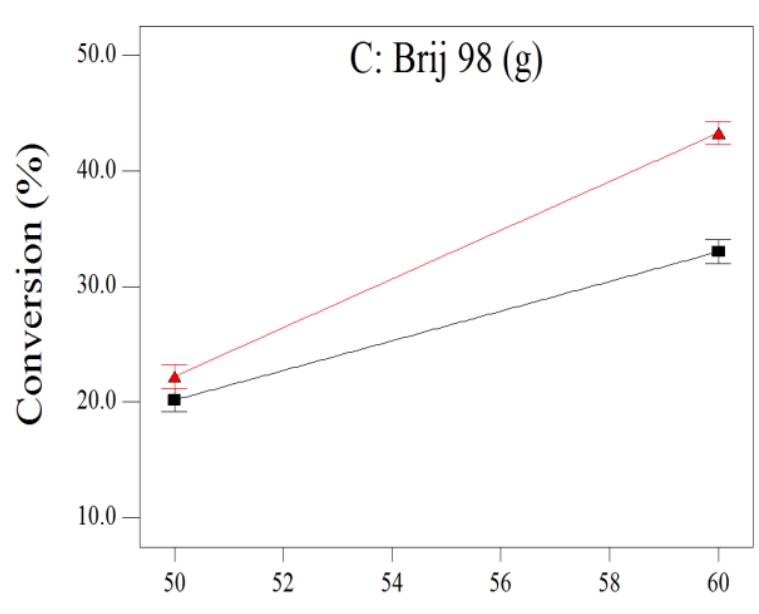

A: Temperature $\left({ }^{\circ} \mathrm{C}\right)$
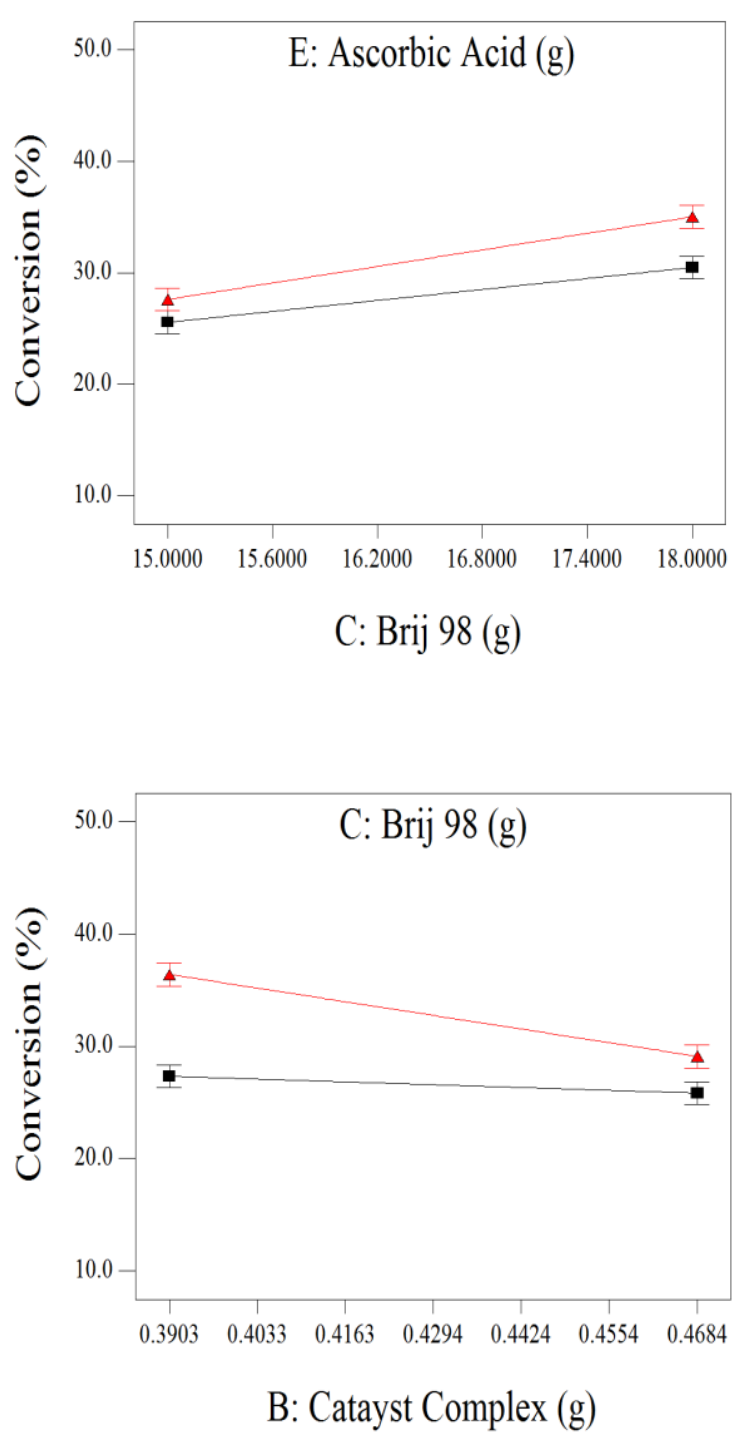

Figure 5.5: Interaction of process inputs and their effects on the conversion 


\subsubsection{Molecular weight}

For the same reasons explained in chapter 5.3.4.1, half normal plot (Figure 5.6) was used to select the terms for the model. Based on the $p$-value (Table 5.4) and from Pareto chart (Figure 5.7), it is found that only A, D, AD and BE are found to be the significant terms, which is clearly evidenced by Bonferroni limit in Pareto chart. However other terms $\mathrm{CD}, \mathrm{AB}, \mathrm{B}, \mathrm{AC}$, $\mathrm{E}, \mathrm{AE}$ and $\mathrm{C}$ are included in the model because of hierarchical reason and for better ANOVA assumptions as evidenced by residual analysis results.

The model $F$-value of 23.70 along with $p$-value of 0.0002 (Table 5.5) proves the significance and validity of the model. However small curvature suggests that the quadratic effects need to be considered if optimization is targeted.

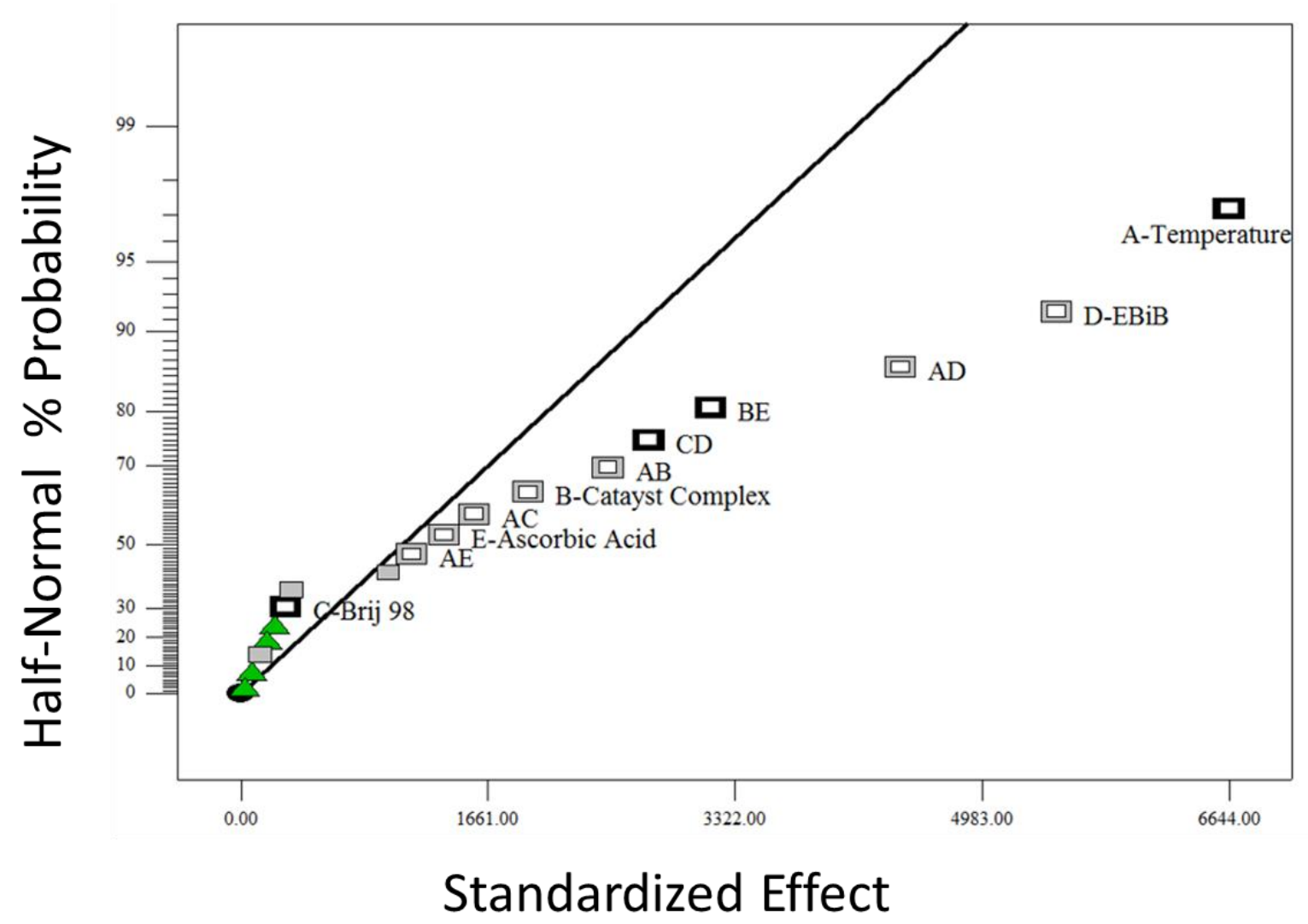

Figure 5.6: Half normal probability plot for the selection of main factors and two factor interaction effects influencing polymer molecular weight. 


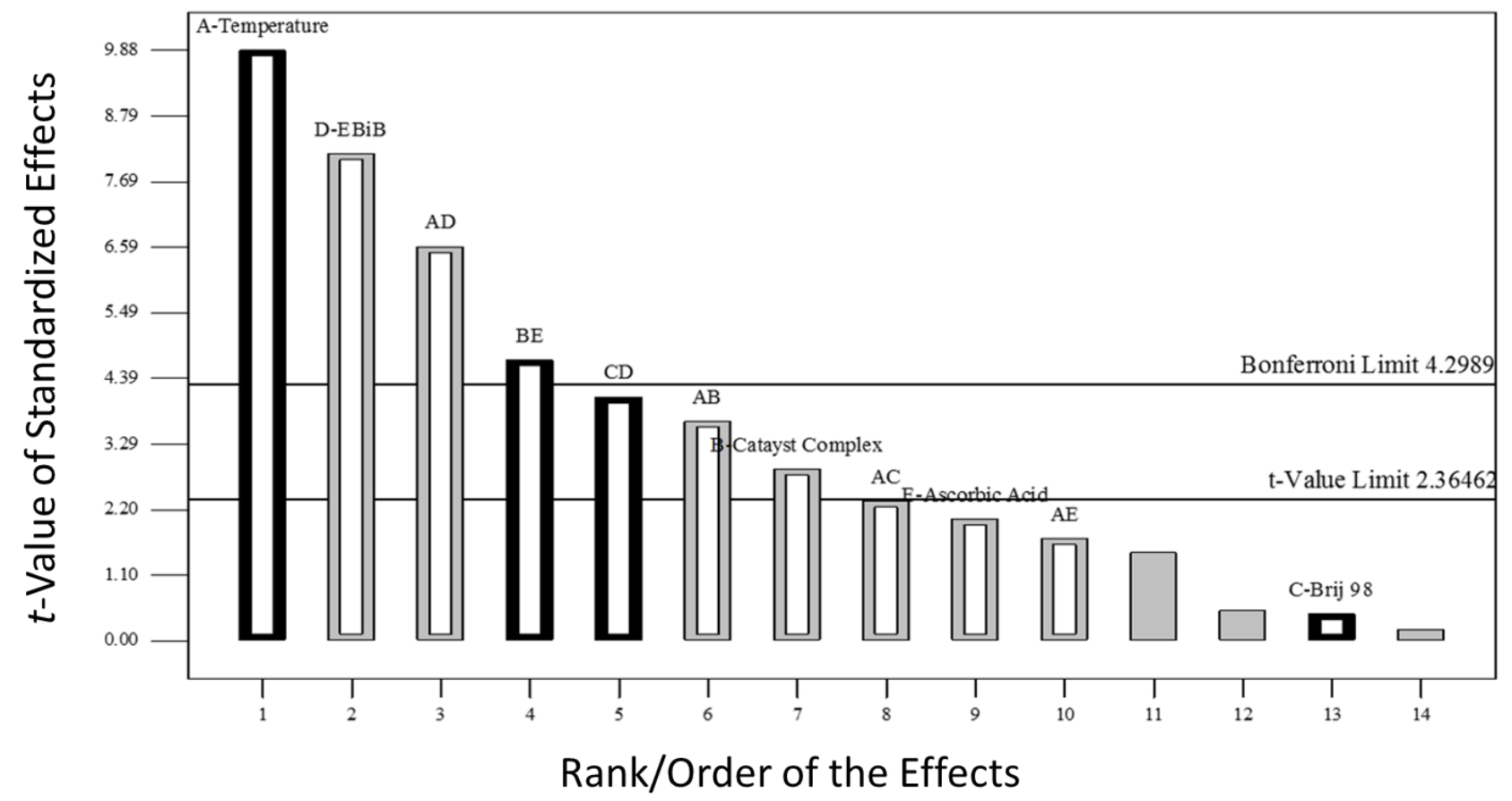

Figure 5.7: Pareto chart with the list of effects according to their influence level on the molecular weight.

Referring Figure 5.8, all diagnostic plots indicate that all the required assumptions of ANOVA are fulfilled. Viewed from Figure. 5.8. (A), all the residuals are close to the straight line; therefore, the normal distribution assumption is satisfied. In Figure 5.8. (B), because all the residual points are scattered randomly all over the graph within the upper and lower bounds instead of accumulating in the other areas, the assumption of constant variance is fulfilled. Figure 5.8. (C), shows that all the residual points are spread within upper and lower bounds, showing no pattern, which verifies the assumption of independence. In Figure 5.8. (D), all the points are close to the straight line, showing that the 'predicted vs actual plot' is satisfactory and the model fits well. 


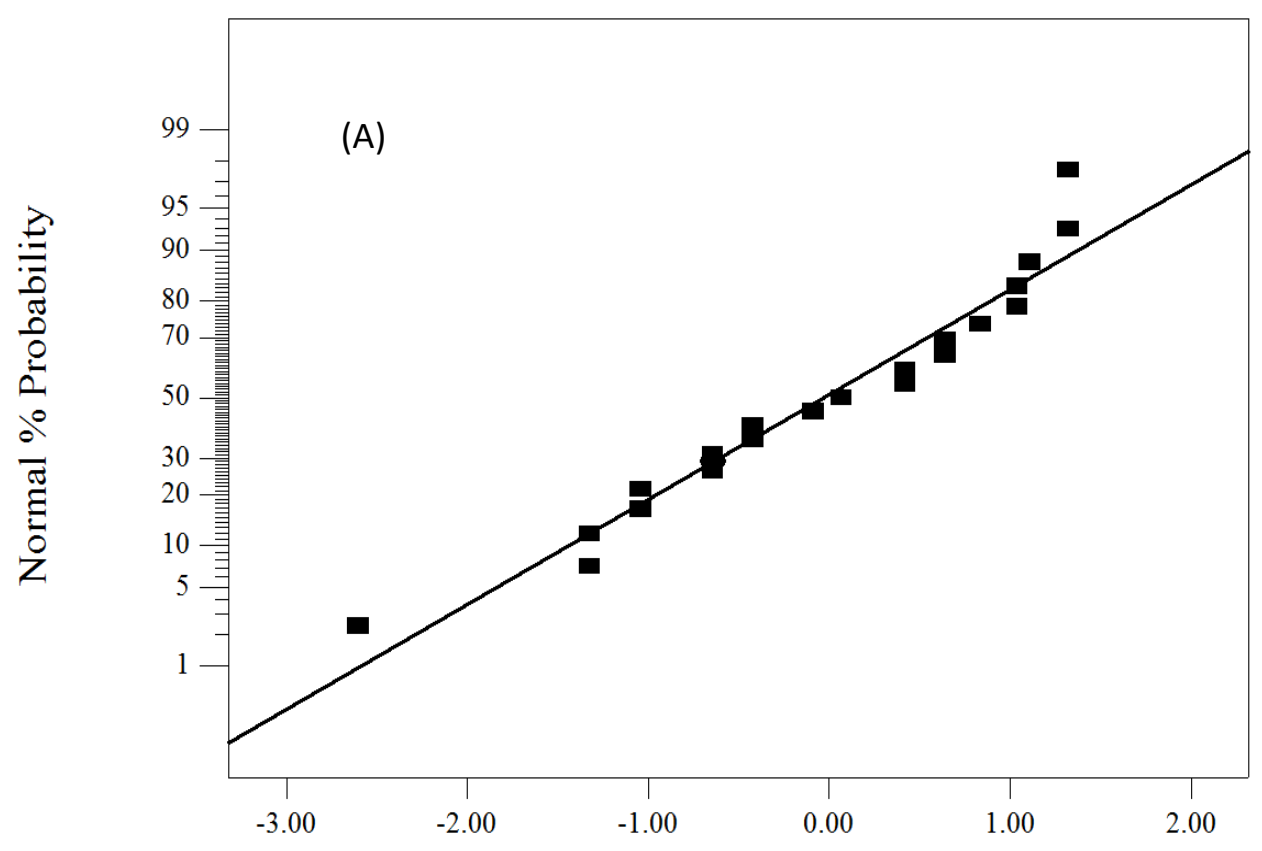

Externally Studentized Residuals

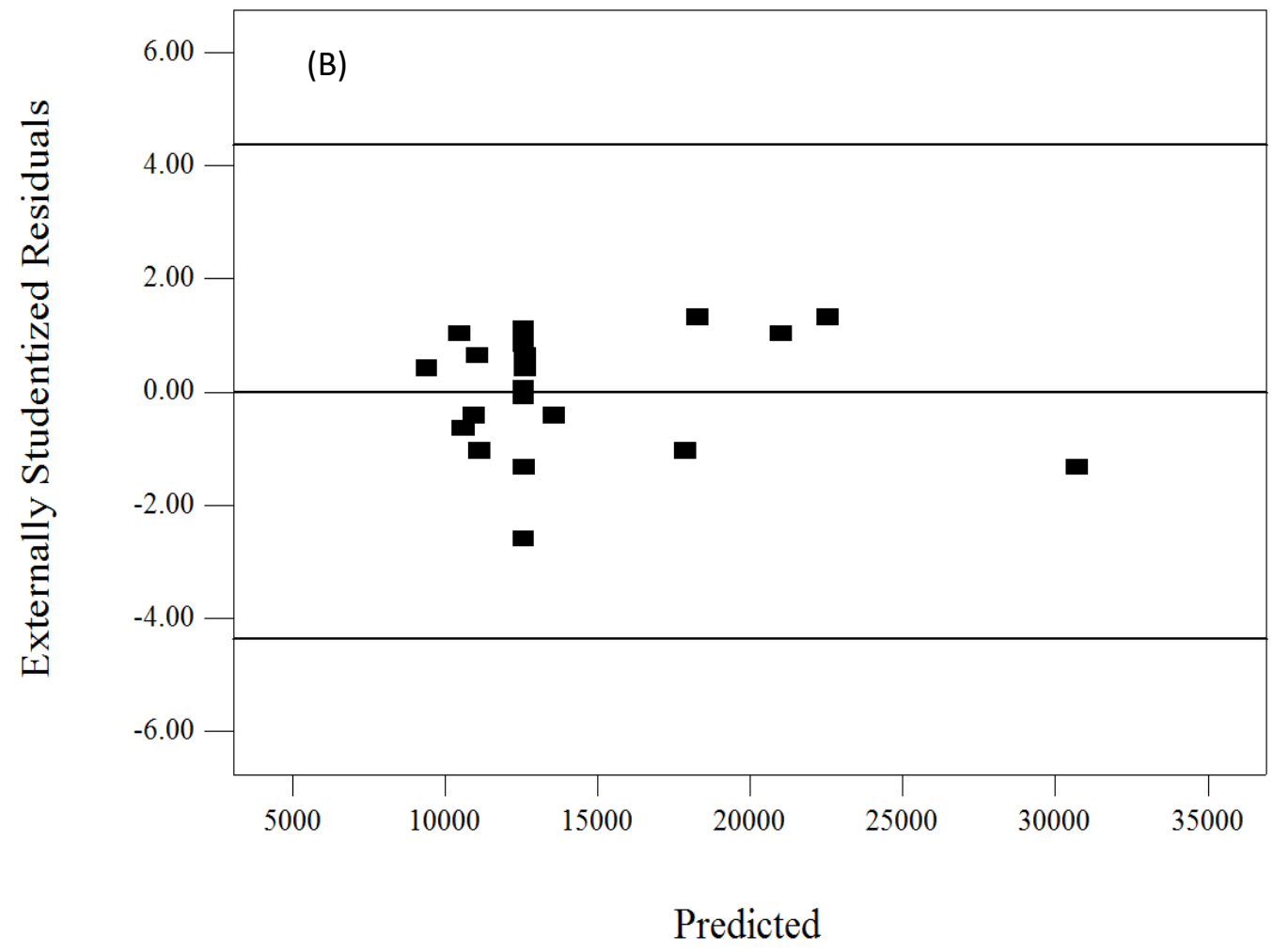




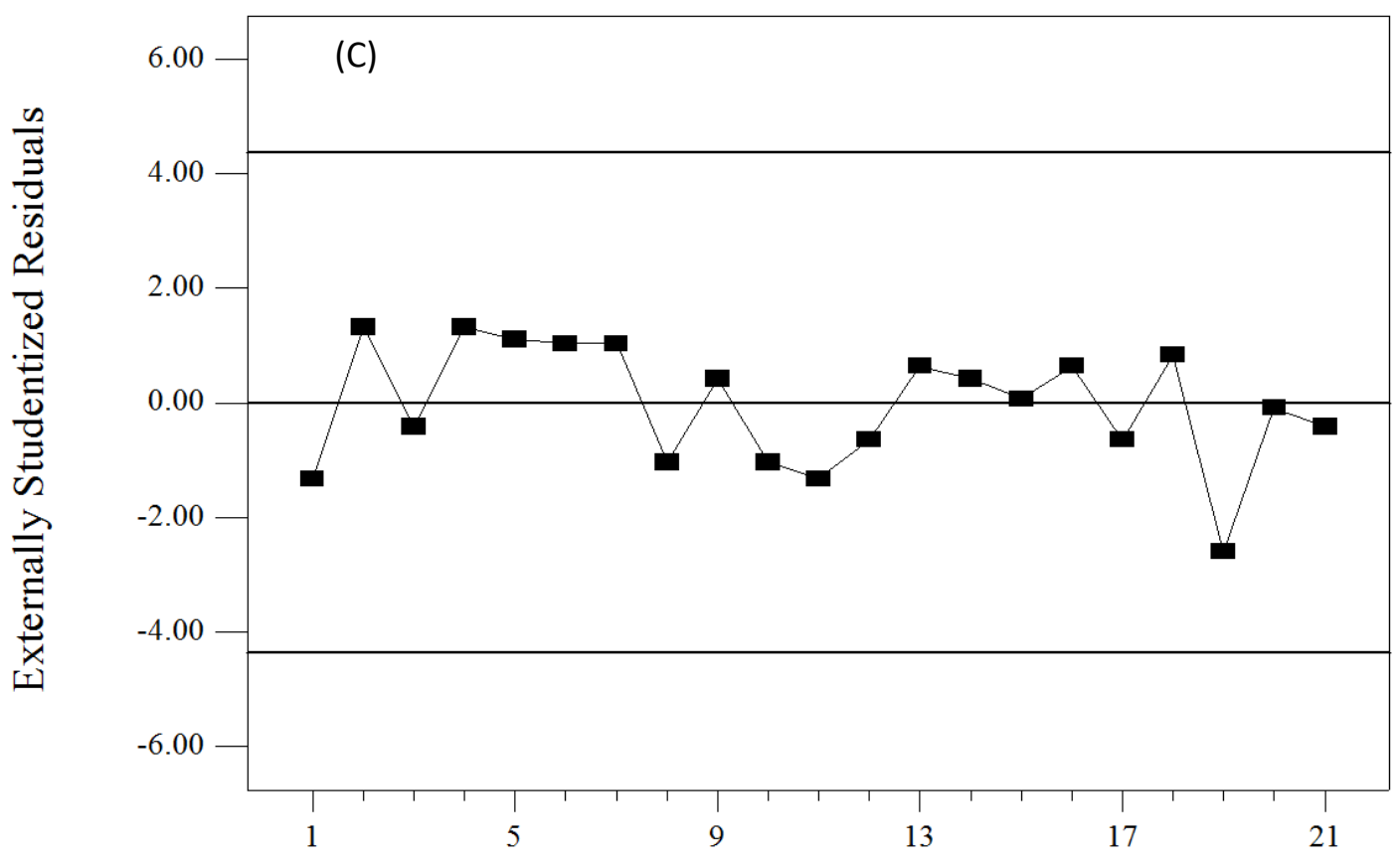

Run Number

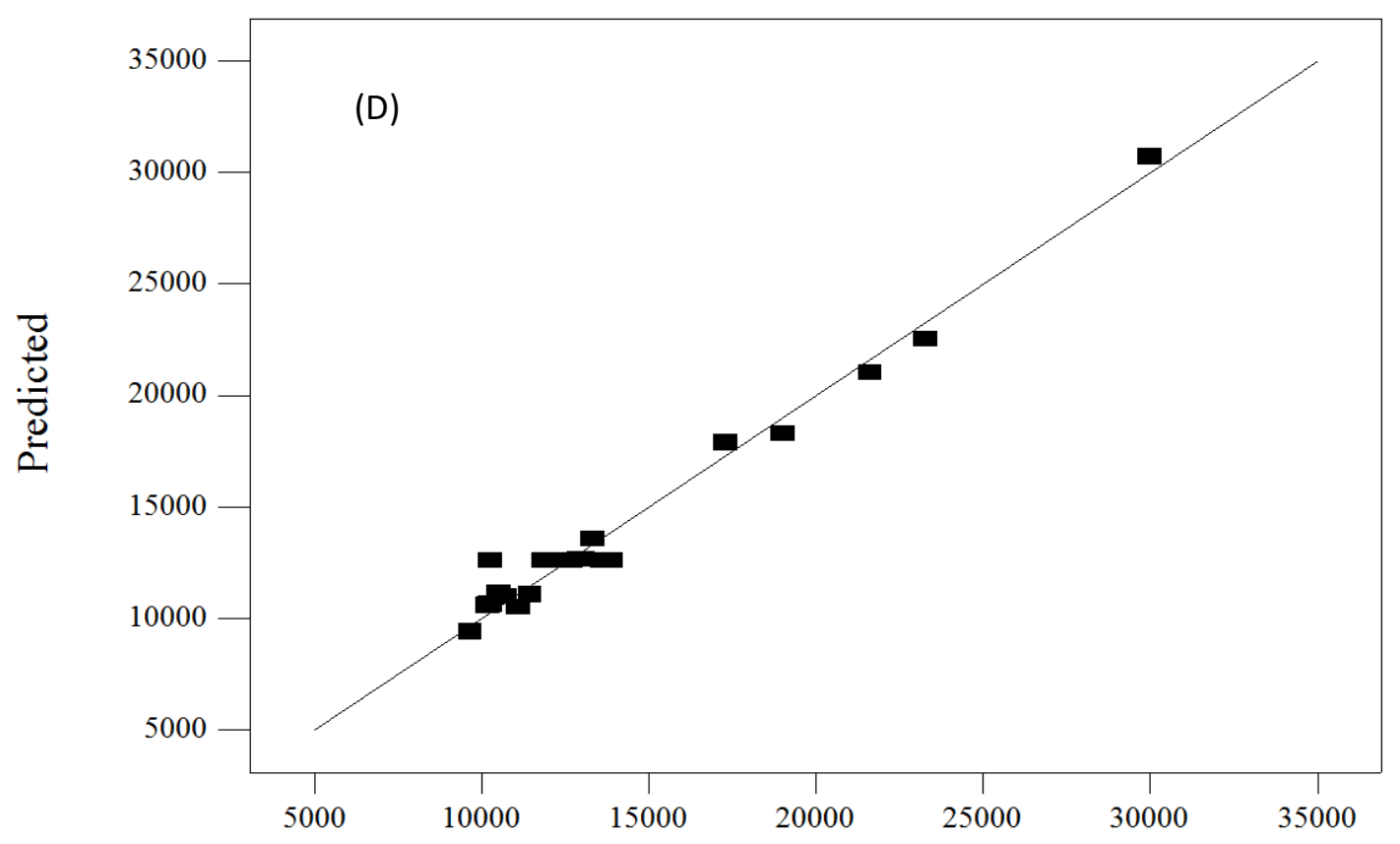

Actual

Figure 5.8: Diagnostic plots for molecular weight: (A) normal probability plot of residuals, (B) residuals vs predicted (C) residuals vs run, and (D) predicted vs actual. 


\section{a) Individual Effects of Independent Variables on Molecular Weight}

The plots shown in Figure 5.9 shows the individual effects of process inputs. Each plot depicts the variation of molecular weight with respect to one key factor while the other four factors were kept invariant at their respective central points. According to Table 5.4, the $p$-values of only two independent variables, reducing agent and surfactant, are more than 0.05 , showing their statistically insignificant influence on the molecular weight.

As can be seen from the Pareto chart (Figure 5.7) and effects list (Table 5.7), temperature has the highest positive influence (32.78\%), followed by the negative influence (22.32\%) of EBiB.

Table 5.7: Effects list showing \% contribution of main factors and two factor interaction effects on molecular weight.

\begin{tabular}{|c|c|c|c|}
\hline Term & $\begin{array}{c}\text { Stdized } \\
\text { Effect }\end{array}$ & $\begin{array}{c}\text { Sum of } \\
\text { Squares }\end{array}$ & $\begin{array}{c}\text { \% } \\
\text { Contribution }\end{array}$ \\
\hline A-Temperature & 6644 & $1.77 \mathrm{E}+08$ & 32.78 \\
B-Catalyst Complex & -1932 & $1.49 \mathrm{E}+07$ & 2.77 \\
C-Brij 98 & 302.75 & $3.67 \mathrm{E}+05$ & 0.068 \\
D-EBiB & -5483.25 & $1.20 \mathrm{E}+08$ & 22.32 \\
E-Ascorbic acid & -1370 & $7.51 \mathrm{E}+06$ & 1.39 \\
AB & -2469.5 & $2.44 \mathrm{E}+07$ & 4.53 \\
AC & -1571.75 & $9.88 \mathrm{E}+06$ & 1.83 \\
AD & -4434.75 & $7.87 \mathrm{E}+07$ & 14.6 \\
AE & -1150.5 & $5.30 \mathrm{E}+06$ & 0.98 \\
BC & -341.25 & $4.66 \mathrm{E}+05$ & 0.086 \\
BD & & Aliased & \\
BE & 3159.5 & $3.99 \mathrm{E}+07$ & 7.41 \\
CD & 2743 & $3.01 \mathrm{E}+07$ & 5.59 \\
CE & -130.75 & 68382.25 & 0.013 \\
DE & -992.75 & $3.94 \mathrm{E}+06$ & 0.73 \\
Curvature & -2130.05 & $1.82 \mathrm{E}+07$ & 3.37 \\
Lack of Fit & & 0 & 0 \\
Pure Error & & $8.18 \mathrm{E}+06$ & 1.52 \\
\hline
\end{tabular}

However, influence of all other independent variables is below $3 \%$. As increasing temperature also increases $\mathrm{K}_{\mathrm{p}}, \mathrm{K}_{\mathrm{act}}$ and $\mathrm{K}_{\mathrm{ATRP}}$ values (Qu et al., 2013; Seeliger and Matyjaszewski, 2009; Tang et al., 2008), it can be inferred that it results in the fast incorporation of monomer units at the active sites causing higher molecular weight at the given time. As the degree of 
polymerization is inversely proportional to the initiator concentration (Braunecker and Matyjaszewski, 2007; Matyjaszewski et al., 1997), molecular weight can be altered by changing the initiator to monomer ratio, which implies that an increase in the initiator concentration decreases the molecular weight.

Though the individual effects of the catalyst complex, reducing agent and surfactant are not very significant, their interaction is clearly important and is discussed below. 

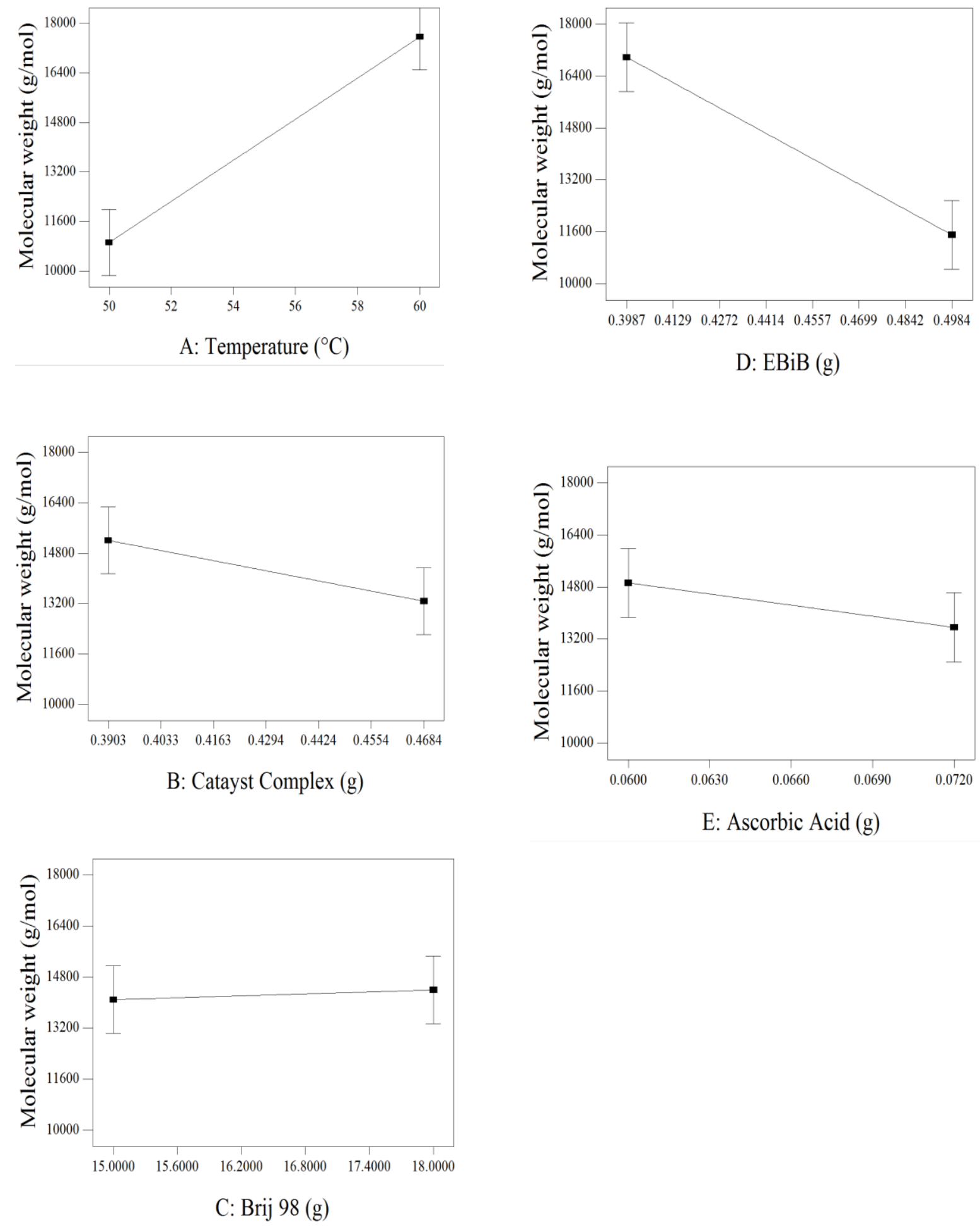

Figure 5.9: Individual effects of process inputs on the average molecular weight of the polymer (PMMA). 


\section{b) Effects of Interactions of Independent Variables on Molecular Weight}

The interaction effect plots are shown in Figure. 5.10. The non-parallel lines in this figure shows an interaction between the two factors. The coefficients in Table 5.4., show 7 negative and 3 positive interactions. However, based on the $p$-value (Table 5.4.), interactions between temperature and initiator (AD) and interactions between catalyst complex and reducing agent (BE) are found to have significant negative and positive impacts on the molecular weight respectively. Despite having a high $p$-value, interactions between surfactant and initiator (CD), and temperature and surfactant (AC) are also important which is also clear from the t-value of Pareto Chart (Figure 5.7). The selection of factors and interactions, despite their high $p$-values in the model is for the better assumption of ANOVA as evidenced by Figure 5.8.

Note: Triangular symbol stands for the high level (+1) and square symbol stands for the low level (-1) of each factor appearing on the top of each plot in Figure 5.10. 


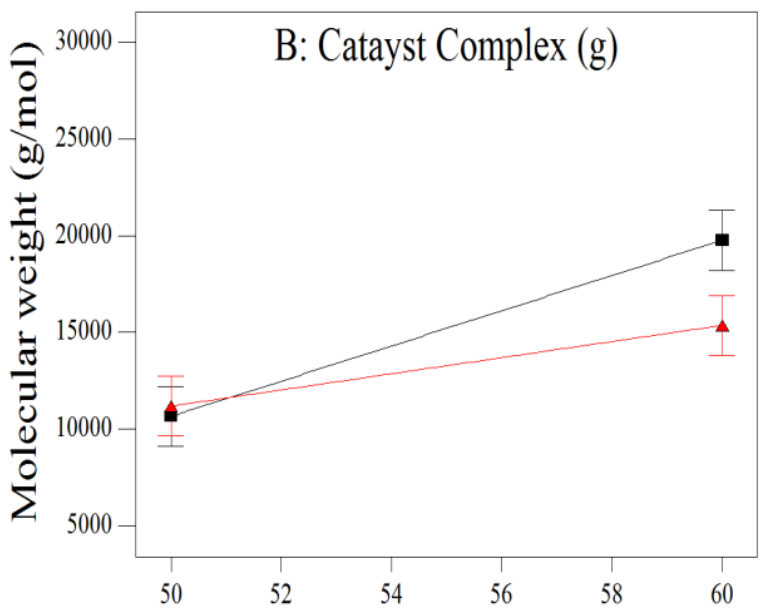

A: Temperature $\left({ }^{\circ} \mathrm{C}\right)$

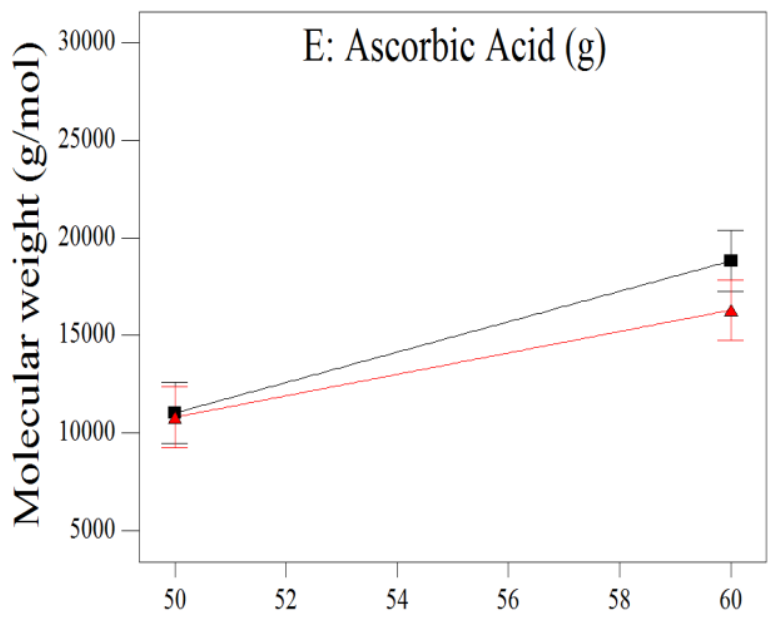

A: Temperature $\left({ }^{\circ} \mathrm{C}\right)$

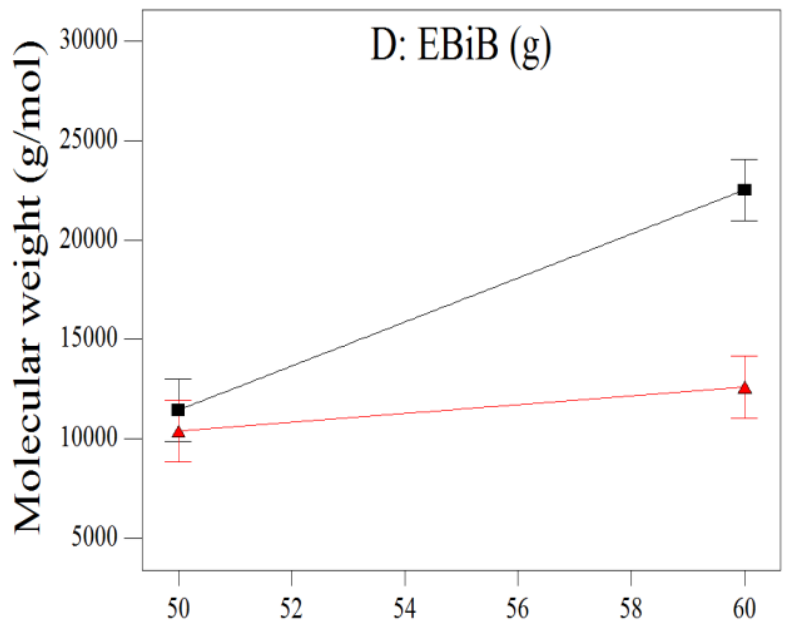

A: Temperature $\left({ }^{\circ} \mathrm{C}\right)$

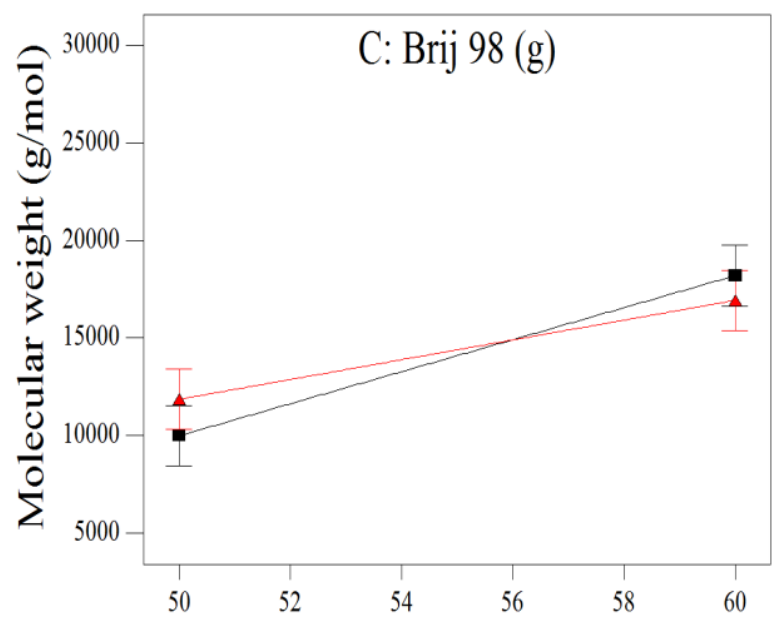

A: Temperature $\left({ }^{\circ} \mathrm{C}\right)$

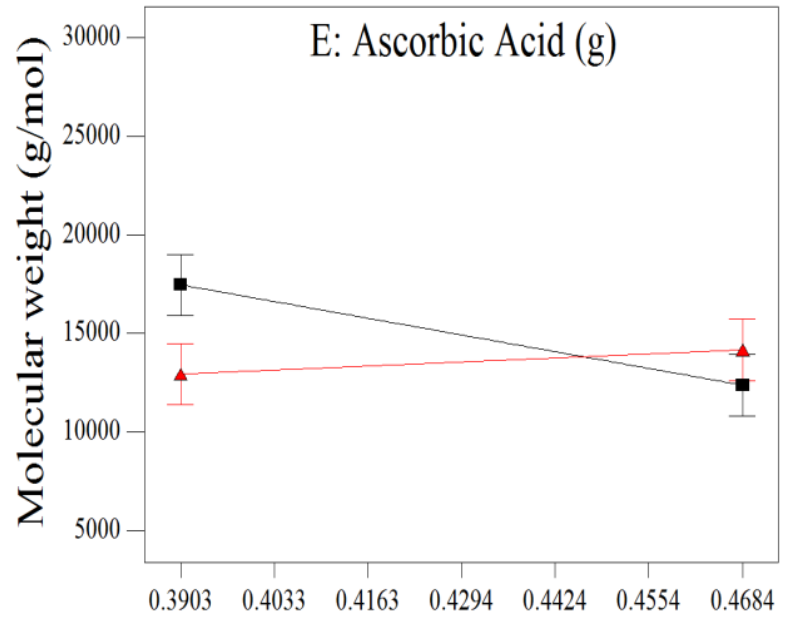

B: Catayst Complex (g)

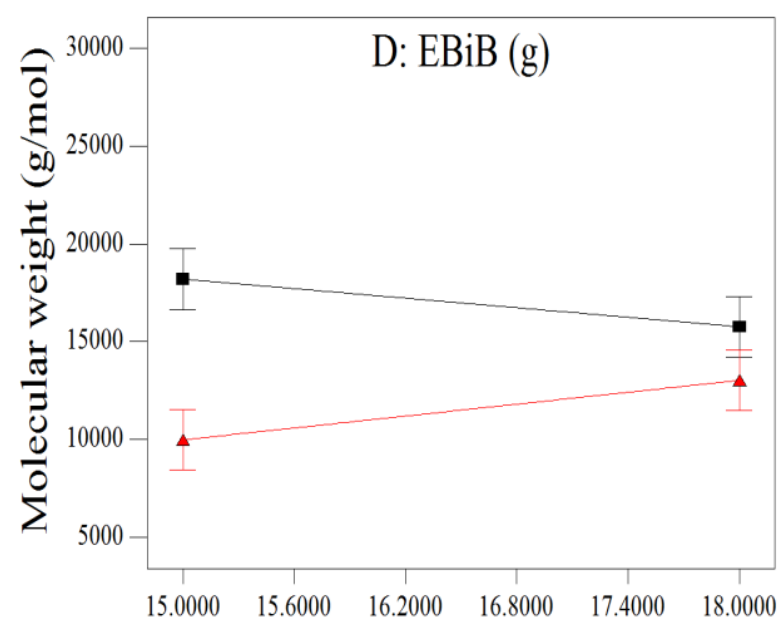

C: Brij 98 (g)

Figure 5.10: Interaction of process inputs and their effect on the average molecular weight of the polymer (PMMA). 


\subsubsection{PDI}

Half normal plot (Figure 5.11) was used for the selection of statistically significant effects. Pareto chart (Figure 5.12) and list of effects (Table 5.8) shows contribution of each effect on the PDI.

The model $F$-value of 121.37 along with $p$-value $<0.00001$ (Table 5.5) proves the significance and validity of the model. However significant curvature suggests that the quadratic effects need to be considered for better prediction (model).

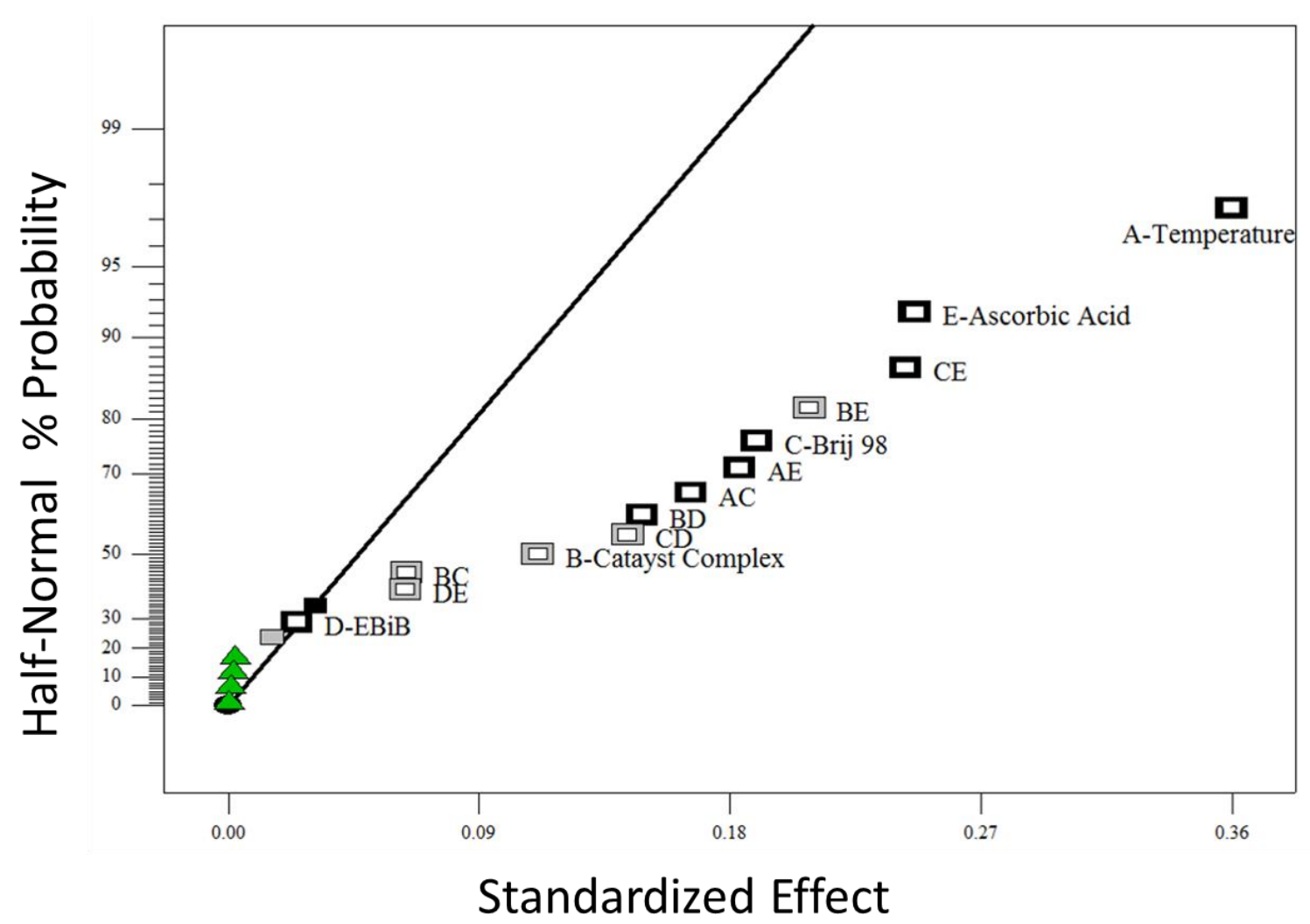

Figure 5.11: Half normal probability plot for the selection of main factors and two factor interaction effects influencing PDI. 
Table 5.8: Effects list showing \% contribution of main factors and two factor interaction effects on PDI.

\begin{tabular}{|c|c|c|c|}
\hline Term & $\begin{array}{c}\text { Stdized } \\
\text { Effect }\end{array}$ & $\begin{array}{c}\text { Sum of } \\
\text { Squares }\end{array}$ & $\begin{array}{c}\text { \% } \\
\text { Contribution }\end{array}$ \\
\hline A-Temperature & 0.36 & 0.52 & 27.76 \\
B-Catalyst Complex & -0.11 & 0.05 & 2.65 \\
C-Brij 98 & 0.19 & 0.14 & 7.7 \\
D-EBiB & 0.025 & $2.45 \mathrm{E}-03$ & 0.13 \\
E-Ascorbic acid & 0.25 & 0.24 & 13 \\
AB & -0.016 & $1.02 \mathrm{E}-03$ & 0.055 \\
AC & 0.17 & 0.11 & 5.9 \\
AD & 0.032 & $3.97 \mathrm{E}-03$ & 0.21 \\
AE & 0.18 & 0.14 & 7.22 \\
BC & -0.064 & 0.016 & 0.88 \\
BD & 0.15 & 0.088 & 4.72 \\
BE & -0.21 & 0.17 & 9.32 \\
CD & -0.14 & 0.082 & 4.41 \\
CE & 0.24 & 0.24 & 12.66 \\
DE & -0.064 & 0.016 & 0.87 \\
Curvature & -0.11 & 0.045 & 2.42 \\
Lack of Fit & & 0 & 0 \\
Pure Error & & $1.92 \mathrm{E}-03$ & 0.1 \\
\hline
\end{tabular}

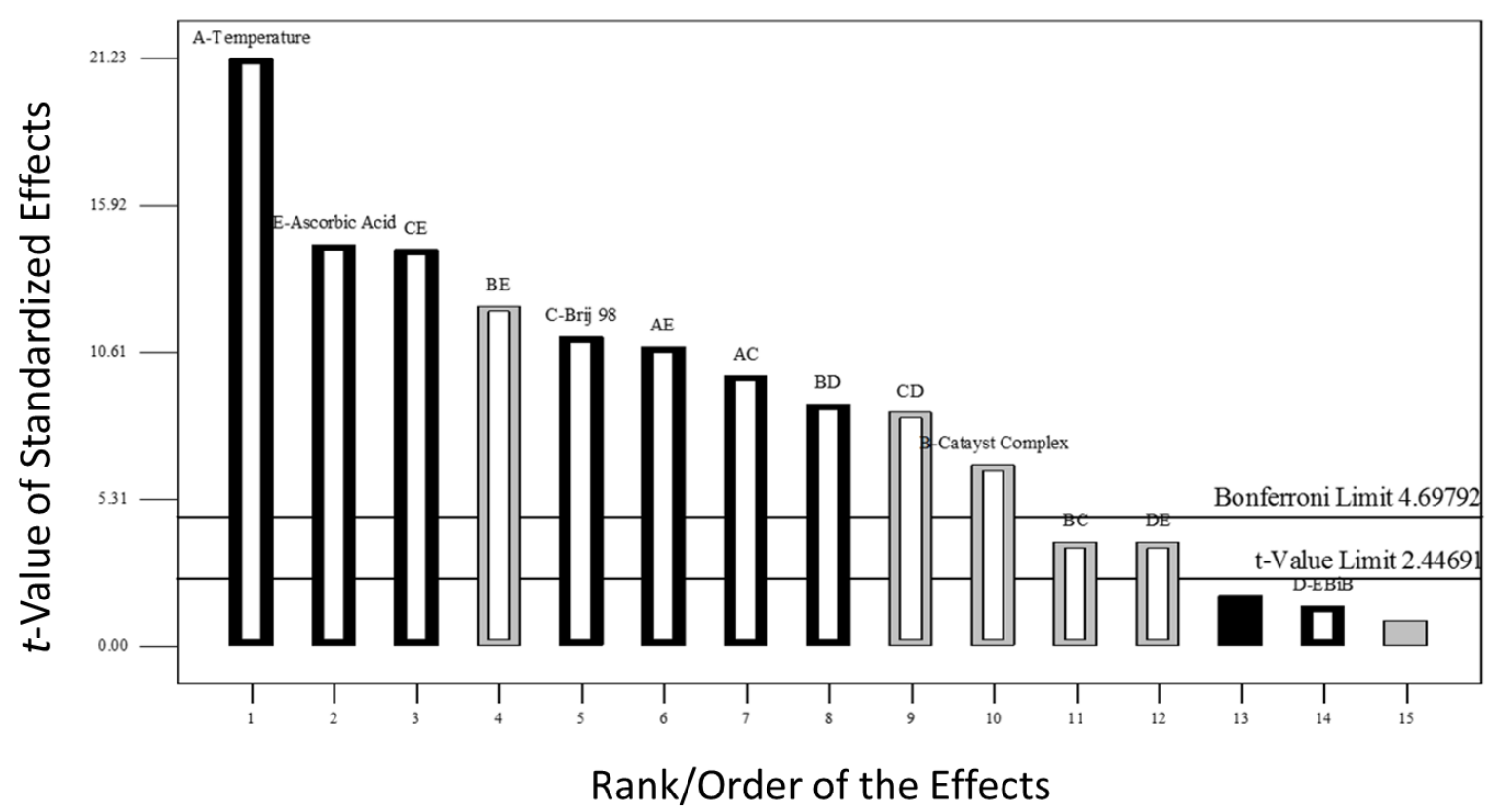

Figure 5.12: Pareto chart with the list of effects according to their influence level on PDI. 
Referring Figure 5.13, all diagnostic plots indicate that all the required assumptions of ANOVA are fulfilled. Viewed from Figure. 5.13. (A), all the residuals are close to the straight line; therefore, the normal distribution assumption is satisfied. In Figure 5.13(B), because all the residual points are scattered randomly all over the graph within the upper and lower bounds instead of accumulating in the other areas, the assumption of constant variance is fulfilled. Figure 5.13. (C), shows that all the residual points are spread within upper and lower bounds, showing no pattern, which verifies the assumption of independence. In Figure 5.13. (D), all the points are close to the straight line, showing that the 'predicted vs actual plot' is satisfactory and the model fits well. 


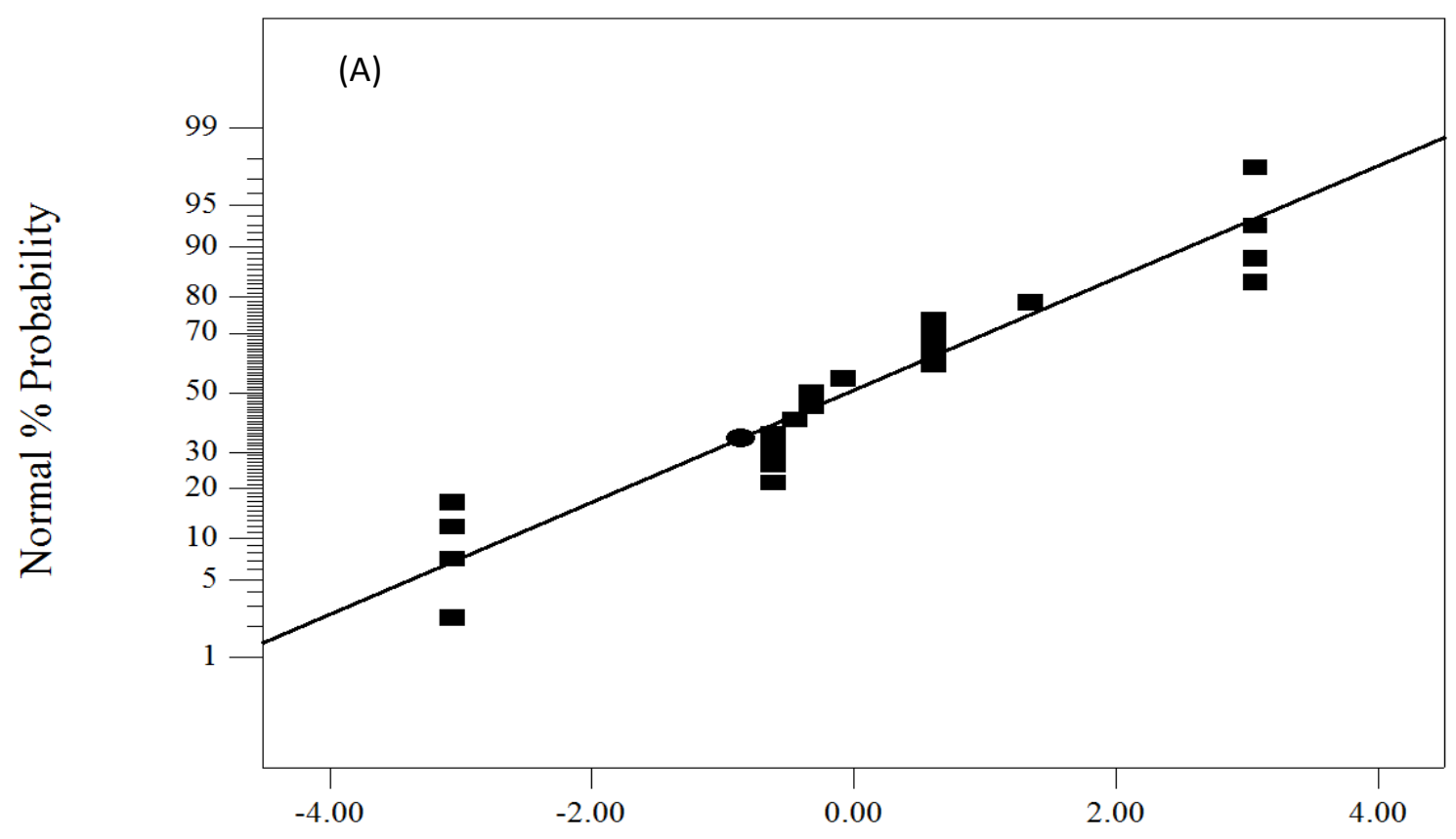

Externally Studentized Residuals

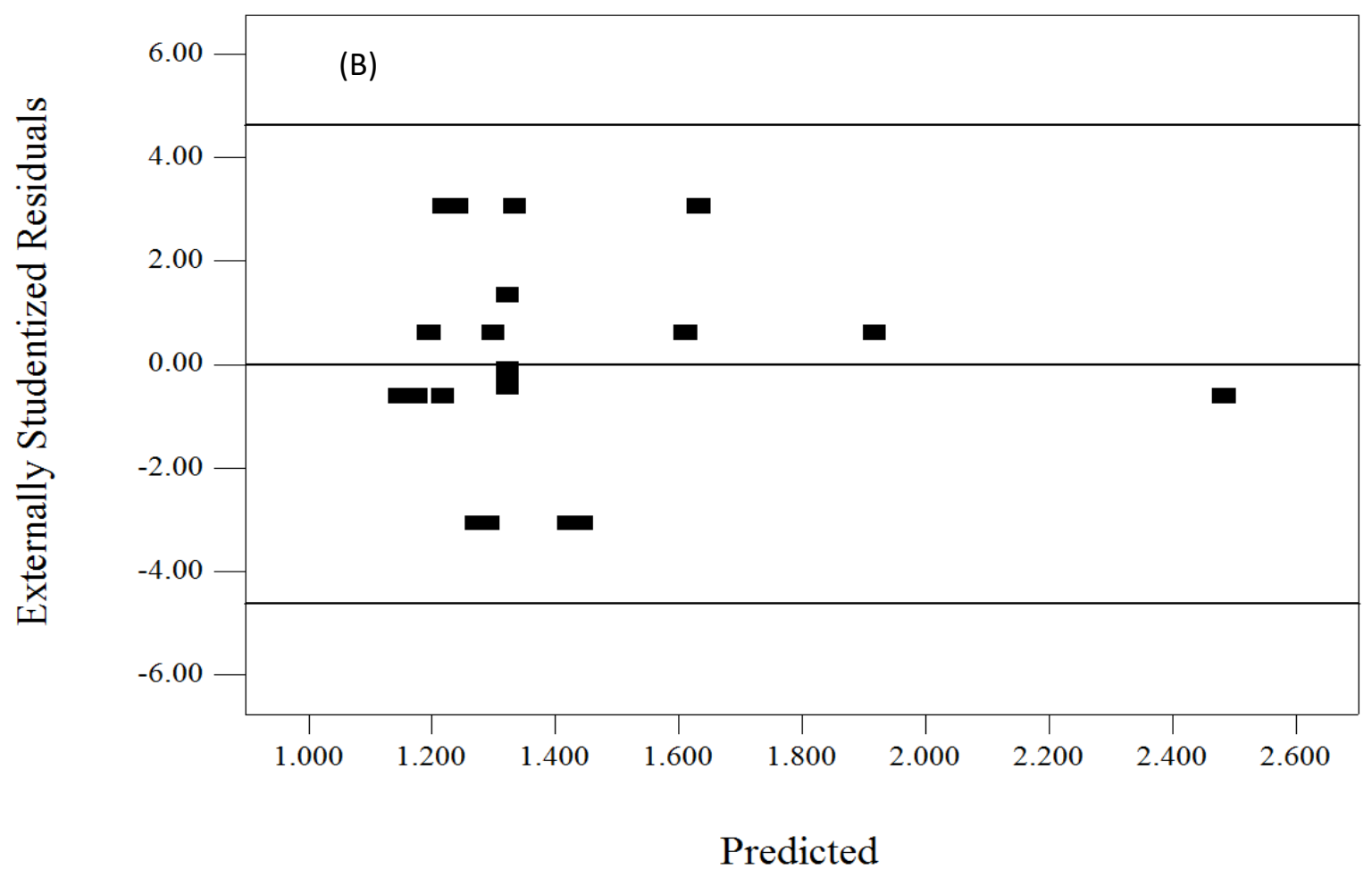




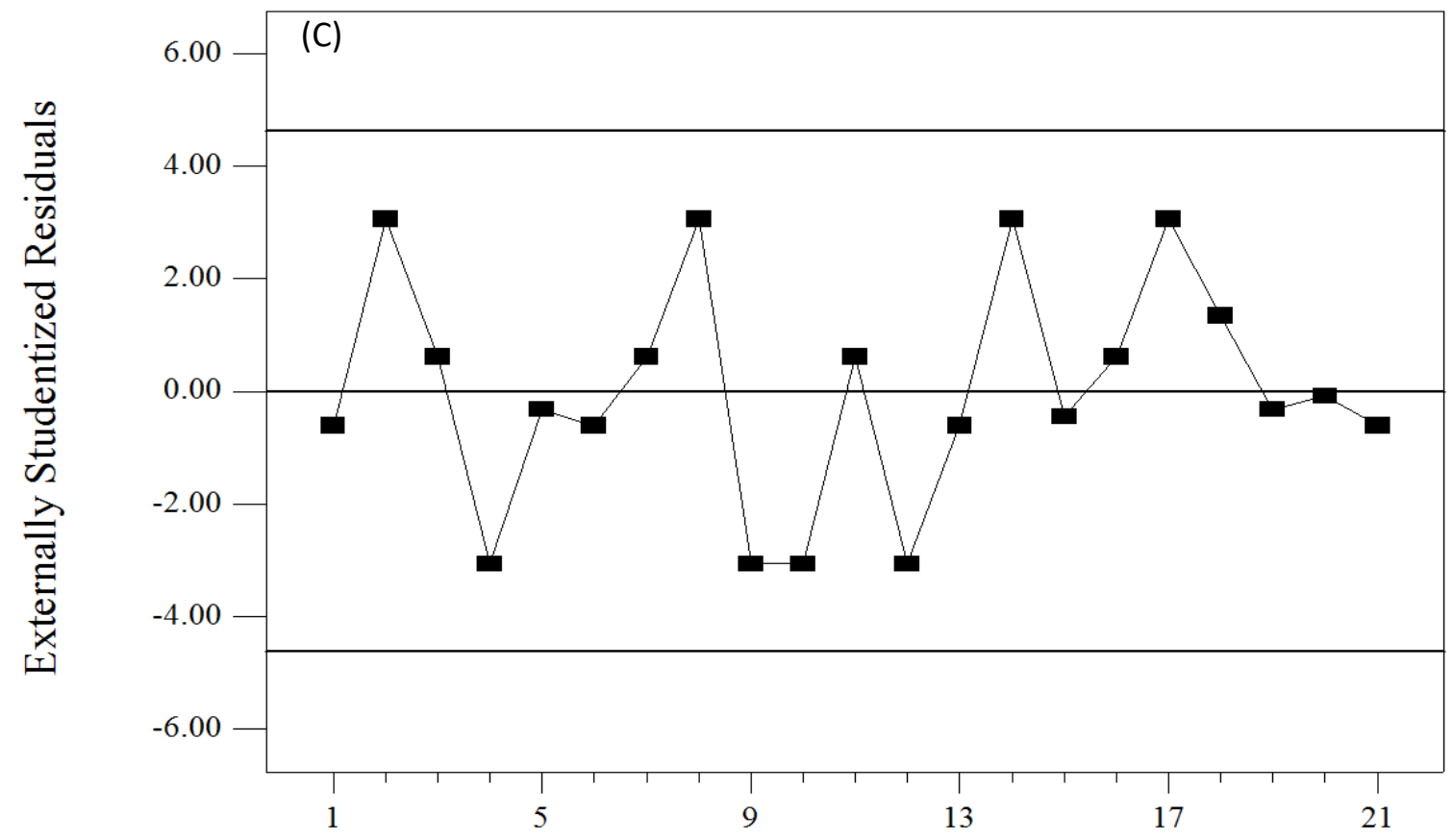

Run Number

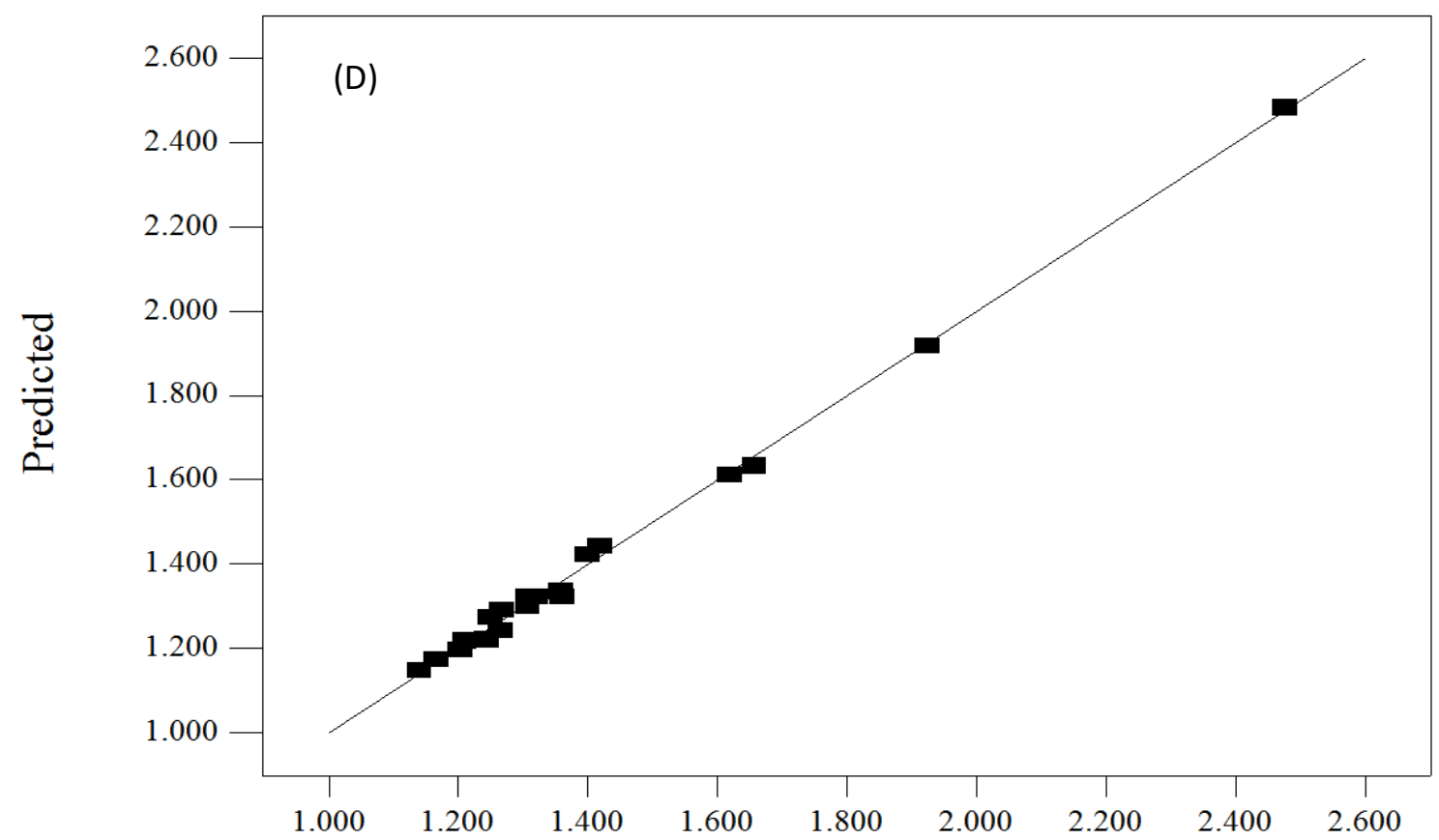

Actual

Figure 5.13: Diagnostic plots for PDI: (A) normal probability plot of residuals, (B) residuals vs predicted, (C) residuals vs run and (D) predicted vs actual 


\section{a) Individual Effects of Independent Variables on PDI}

The plots shown in Figure 5.14., show the individual effects of process parameters on PDI. Each plot depicts the variation of PDI with respect to one key factor while the other four factors were kept invariant at their respective central points. Based on the $p$-value (Table 5.4) it is found that the initiator is not important on its own, however its interaction with other factors is important for PDI.

As can be seen from the Pareto chart (Figure 5.12) and effects list (Table 5.8), temperature has the highest positive influence $(27.76 \%)$, followed by reducing agent $(13 \%)$, surfactant $(7.7 \%)$ and initiator $(0.13 \%)$. Catalyst complex has highest negative influence $(2.65 \%)$.

At higher temperatures, side reactions (chain transfer) can become pronounced (Qu et al., 2013) which can result in higher PDI. Further, at higher temperature loss of control can also result in high PDI.

According to Equation 2.2, it can be said that smaller amounts of deactivator in the system can cause broad PDI. An increased concentration of reducing agent converts more deactivators into activators, hence high PDI is expected. However, the proportion of reducing agent with catalyst complex is more important for the control of the polymerization and hence PDI. Again from the same Equation it can also be inferred that increased deactivator in the system decreases, which is evidenced by the negative effect of the catalyst complex on PDI (Figure 5.14).

With higher amount of surfactant, it is more likely that there will be more polymerization loci with different activator and deactivator ratio resulting into different rate of polymerization and hence broad PDI.

Negligible effect of initiator on PDI can again be blamed on the very high initiator to catalyst molar ratio, where a change in initiator concentration does not make much change in the PDI, as the catalyst complex is always lower in number than the chains that can propagate. Otherwise increased initiator concentration should increase PDI, as indicated by Equation (2.2). Very small positive effect of initiator on PDI is obvious in Figure 5.14. 


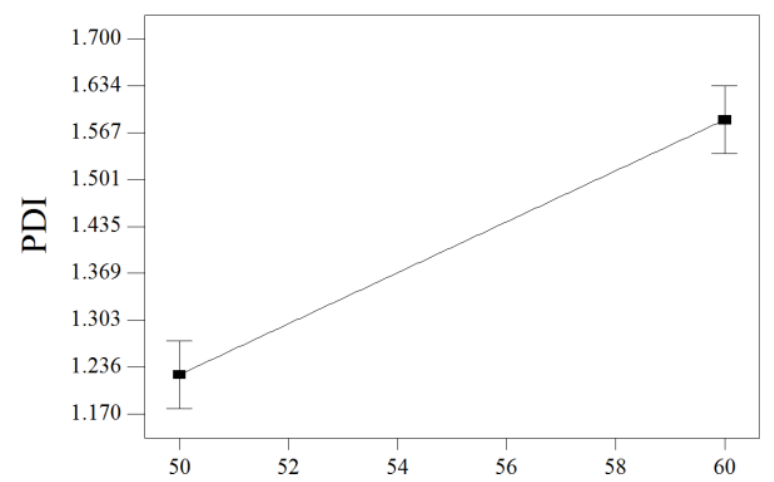

A: Temperature $\left({ }^{\circ} \mathrm{C}\right)$
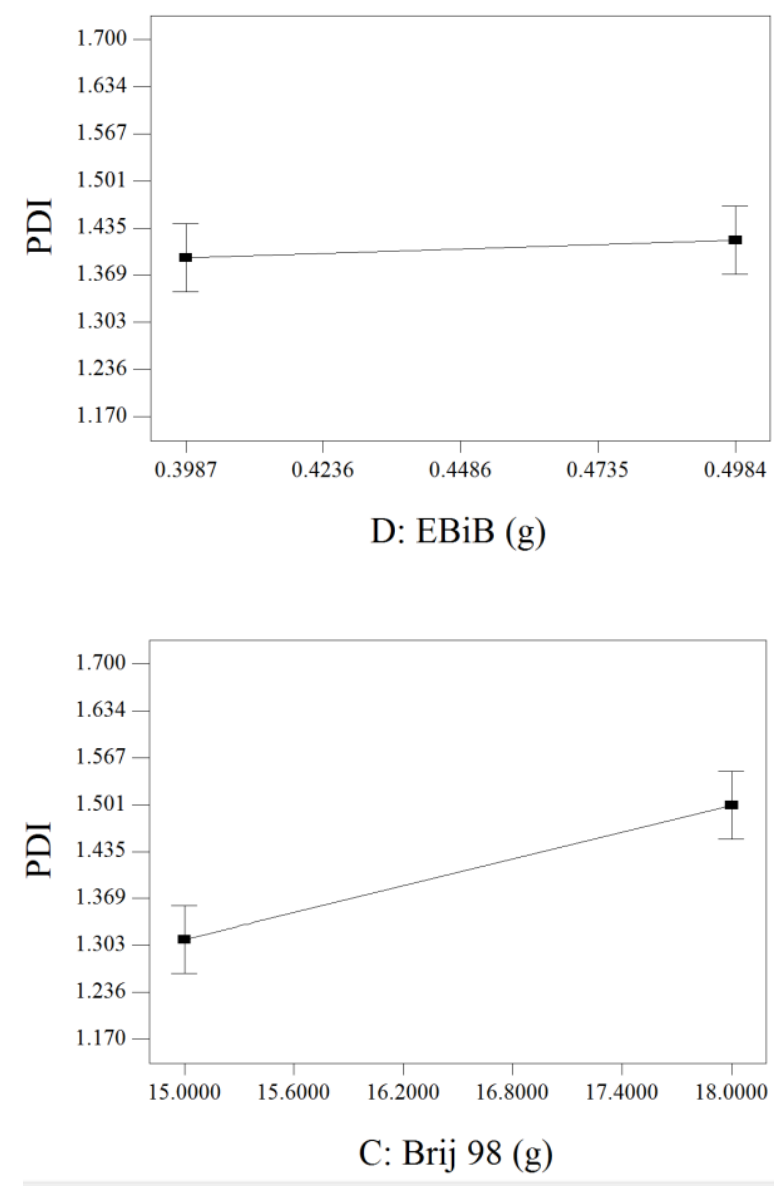
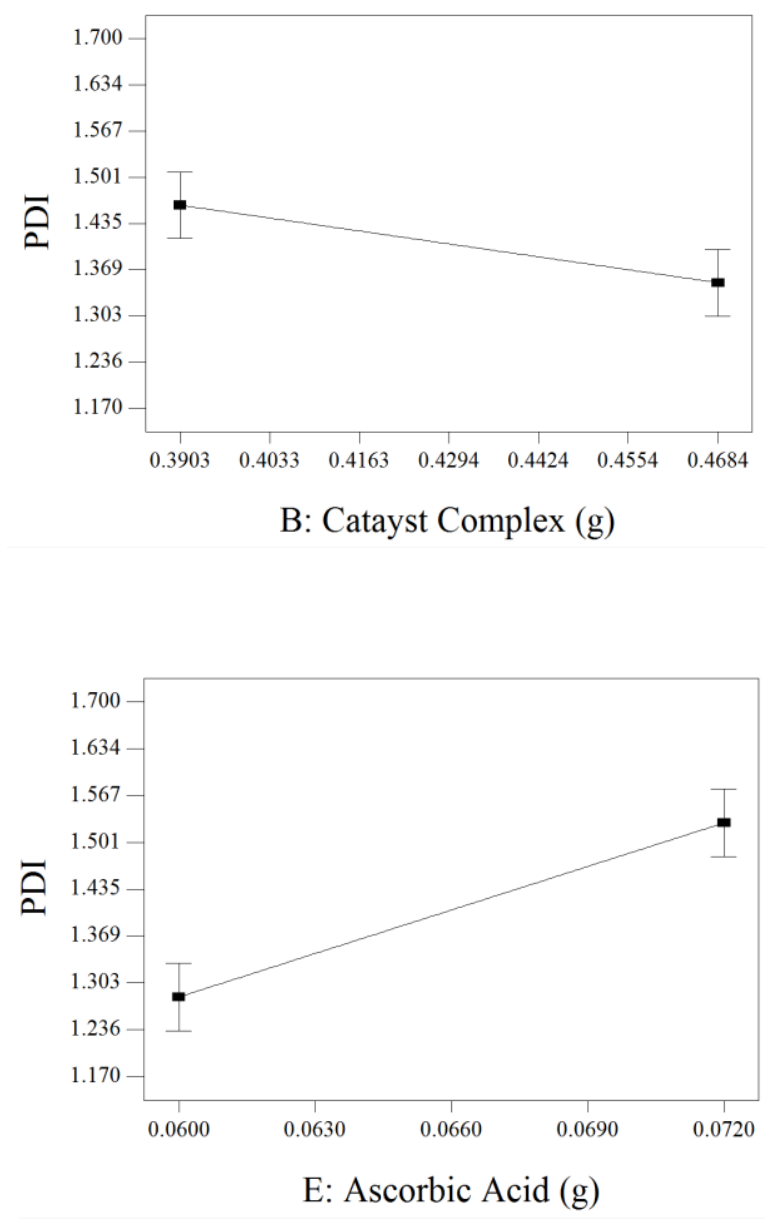

Figure 5.14: Individual effects of process inputs on the PDI 


\section{b) Effects of Interactions of Independent Variables on PDI}

The interaction effect plots are shown in Figure. 5.15. The non-parallel lines in this Figure show an interaction between the two factors. The coefficients in Table 5.4., shows 5 positive and 5 negative interactions. However, based on the $p$-value, only the interaction between temperature and catalyst complex $(\mathrm{AB})$ is found to be statistically insignificant. However, based on the effect list (Table 5.8) it can be said that interactions CE, BE, AE, AC, BD and CD are more important. Along with the significant individual effect, reducing agent affects PDI in multiple ways through the significant interaction with surfactant $(\mathrm{CE})$, catalyst complex $(\mathrm{BE})$, temperature (AE) and initiator (DE). However, interaction between catalyst complex and reducing agent $(\mathrm{BE})$ has most significant negative impact and interaction between surfactant and reducing agent has most significant positive impact on PDI.

Note: In Figure 5.15 triangular symbol stands for the high level $(+1)$ and square symbol stands for the low level (-1) of each factor appearing on the top of each plot. 


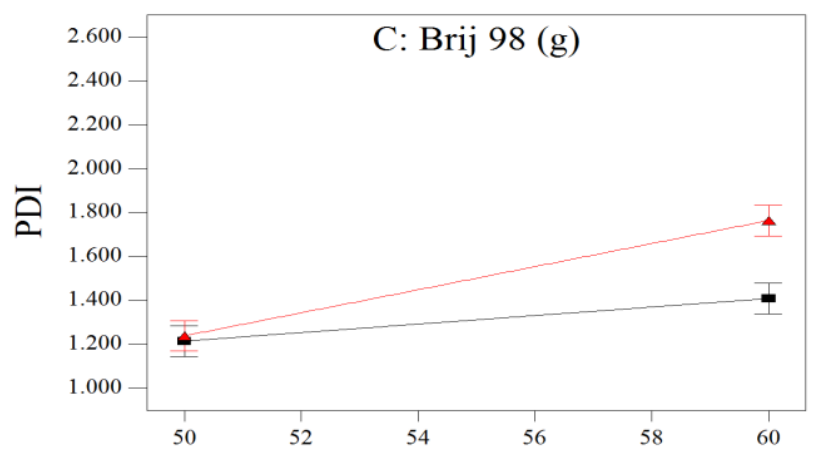

A: Temperature $\left({ }^{\circ} \mathrm{C}\right)$
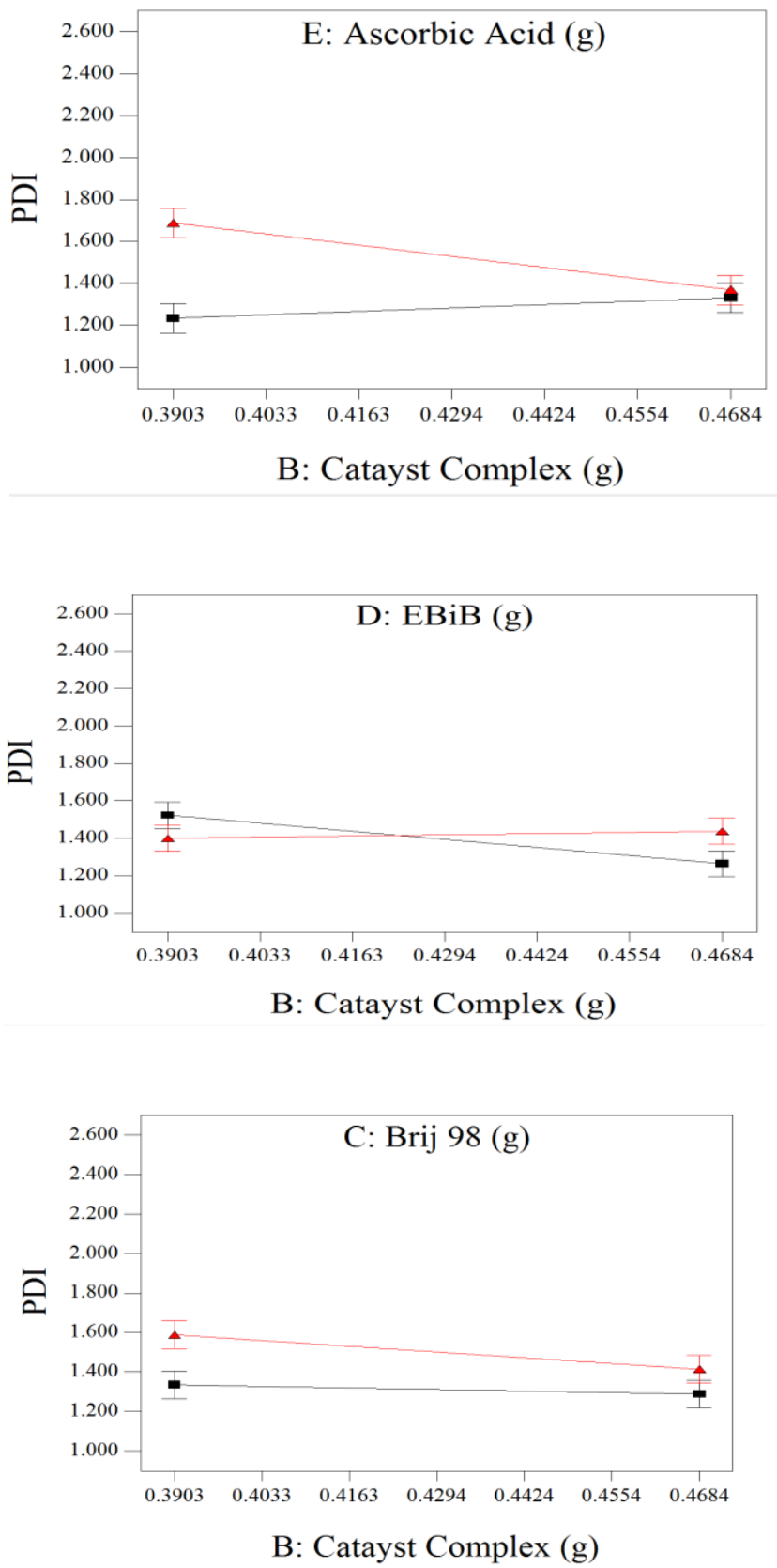

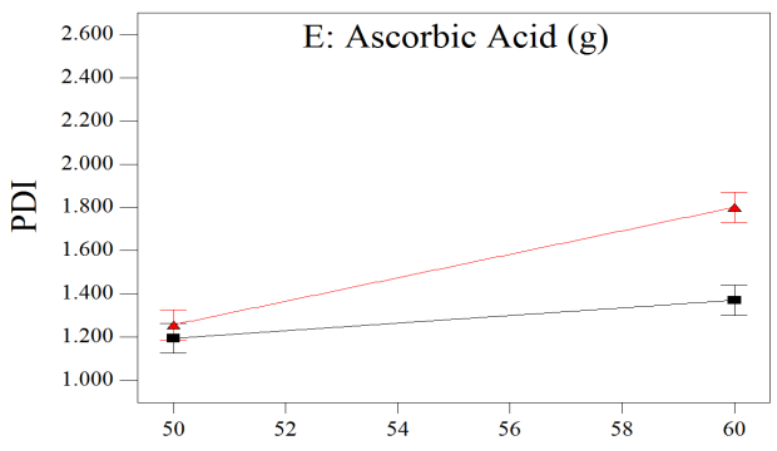

A: Temperature $\left({ }^{\circ} \mathrm{C}\right)$
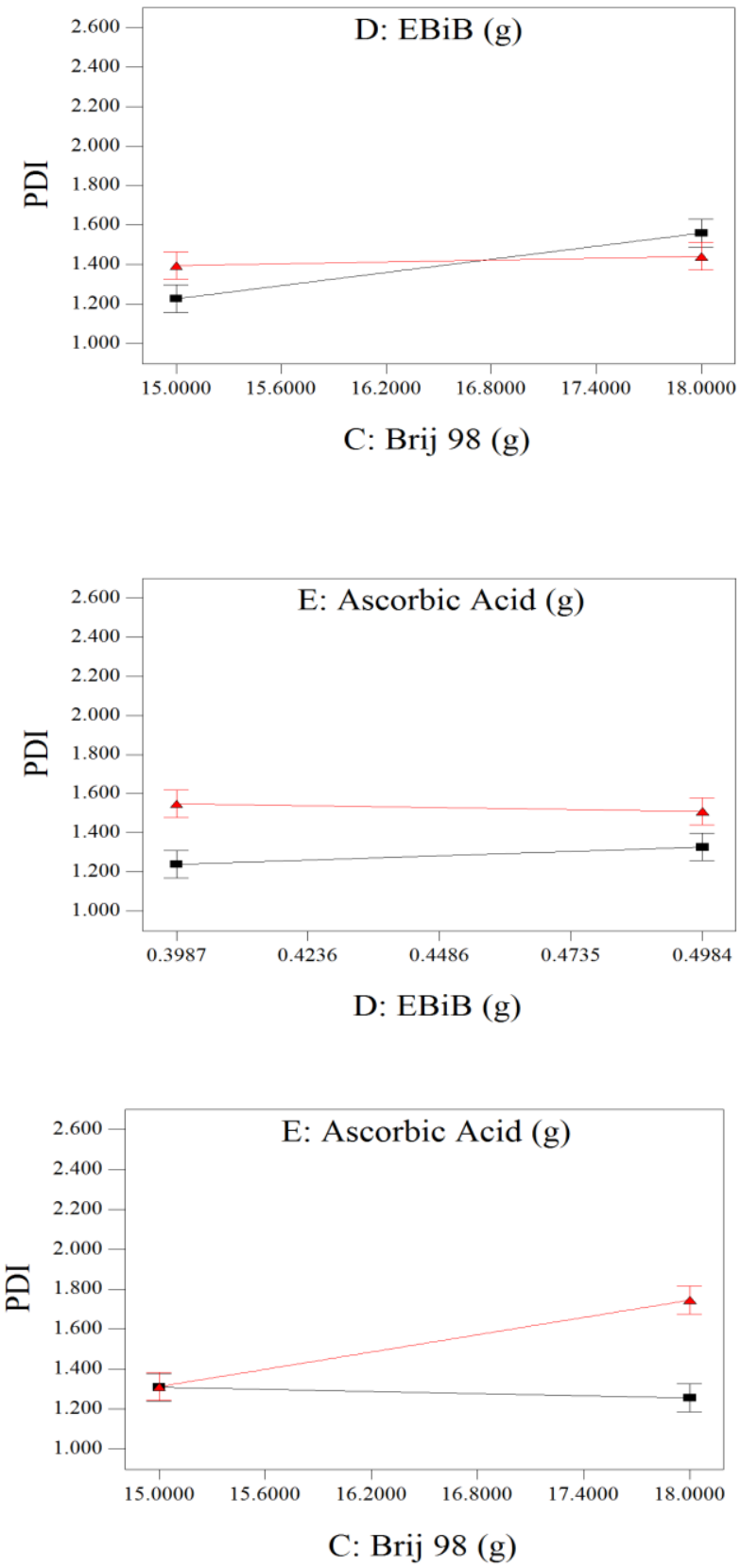

Figure 5.15: Interaction of process inputs and their effect on the PDI 


\subsection{Concluding Remarks}

In this chapter, the two-step emulsion AGET ATRP of MMA was investigated in the 2-L stirred tank reactor. Resolution 5 fractional factorial design $\left(2{ }_{\mathrm{v}}{ }^{5-1}\right)$ was employed to set a design of experiments and determine the most significant factors and their interaction effects on the process variables, which are: conversion, PMMA average molecular weight and PDI. Out of the five independent variables, reaction temperature is found to be the most influential factor for all three process variables with $71.34,32.78$ and $27.76 \%$ contribution to the conversion, molecular weight and PDI, respectively. Next, the surfactant, initiator and reducing agent are found to be influential factors for conversion, molecular weight and PDI, respectively. However, the initiator is the least influential factor for both conversion and PDI, whereas the surfactant is the least influential factor for the PMMA average molecular weight. For all three response variables, the temperature has positive influence, whereas the catalyst complex has a negative one. On assessing the interaction effects, the interaction between the reaction temperature and surfactant is most important for conversion, however, the interaction between the temperature and initiator is the most important for molecular weight. Interactions between surfactant and ascorbic acid mostly affect the PDI of the polymers. 


\section{CHAPTER 6}

\section{CONCLUSIONS AND RECOMMENDATIONS}

The research topic (AGET ATRP of MMA in emulsion system) undertaken in this thesis is relatively new and it is still largely unstudied area, especially in chemical reactors of regular sizes. In this last chapter of the thesis, the results obtained in this study are concisely summarized and few recommendations are outlined for future work.

\subsection{Conclusions}

Emulsion ATRP polymerization of methyl methacrylate (MMA) was thoroughly investigated in a 2-L stirred tank reactor using activators generated by electron transfer (AGET) as the initiation technique. The most important concluding remarks are as follows:

1. The topic studied in this thesis is considered a novel research investigation since no similar study has been previously done on AGET ATRP of a vinyl polymer in a dispersed media in a reactor of 2-L or even closer size. Most if not all ATRP polymerization have been done in small reactors and bulk/solution medium. Therefore, so far there is no guidelines to perform successful experiments. The first part of this thesis was to establish a reliable experimental procedure.

2. A set of experiments were performed according to single-step and two-step procedures in the presence of limited amounts of air using commercially available chemicals: copper bromide/4,4'-dinonyl-2,2'-dipyridyl ( $\left.\mathrm{CuBr}_{2} / \mathrm{dNbpy}\right)$ as the catalyst complex and polyoxyethylene (20) oleyl ether (Brij 98) as the surfactant. Ascorbic acid (AA) and ethyl-2bromoisobutyrate $(\mathrm{EBiB})$ were used as the reducing agent and initiator respectively. The aim of the study was to investigate the feasibility and limitations of each experimental procedure: the single-step and two-step emulsion polymerizations to produce PMMA. Then, to determine and use the most appropriate method of the two procedures for a good design of experiments and statistical analysis for process identification. In one hand, the screening results showed that aggregation/coagulation is a serious issue in both procedures. For a single-step procedure with high conversion and good latex stability, the controlled/living feature was more compromised but for limited experimental conditions only. On the other hand, the controlled/living features of AGET ATRP of MMA were preserved much better in two-step than in single-step 
procedures. However, it is quite challenging to get simultaneously high conversion and good latex stability in the two-step procedure. In overall, the two-step procedure was found to be more promising than single-step procedure.

3. Preliminary experimental investigation served as experimental evidence to opt for a twostep emulsion polymerization. Consequently, a series of experimental tests based on the twostep procedure were carried out to determine the main effects and two factor interaction effects of the temperature, surfactant, catalyst complex, initiator and reducing agent on the monomer conversion, polymer molecular weight and polydispersity index (PDI). Two level fractional factorial design with resolution five $\left(2_{\mathrm{v}}{ }^{5-1}\right)$, was adopted to design the set of experiments required to determine the impact of operating variables on the response variables. An inputoutput process identification model was developed using the FFD procedure. The quality of the model was assessed using statistical analysis.

4. The results of experimental data and statistical analysis revealed that temperature is the most influential factor for all three process responses with $71.34,32.78$ and $27.76 \%$ contribution to the conversion, molecular weight and PDI respectively. Beside temperature surfactant, initiator and reducing agent are found to be the most important contributing factors for conversion, molecular weight and PDI respectively. The initiator is the least influential factor for both conversion and PDI, whereas the surfactant is the least influential factor for molecular weight. For all three response variables temperature has a positive influence and the catalyst complex has a negative influence. Among the interaction effects, interaction between temperature and surfactant is most important for conversion, interaction between temperature and initiator is most important for molecular weight and interaction between surfactant and ascorbic acid is most important for PDI.

\subsection{Recommendations for Future Work}

Based on the results achieved in this study, the following topics are suggested for future work.

1. To investigate two-step emulsion procedure using more appropriate ligand BPMODA instead of dNbpy to achieve higher conversion without coagulation/aggregation.

2. To account for the curvature detected in the model of PDI and molecular weight and for the purpose of optimization use of response surface methodology (RSM), selecting 
most significant factors: temperature, surfactant and reducing agent is highly recommended.

3. Investigation of the emulsion ATRP of MMA in industrially relevant semi-batch or CSTR system is highly desirable. 


\section{APPENDICES}

\section{Appendix A: Recent Developments in ATRP}

Table A.1: ATRP Methods based on initiation techniques.

\begin{tabular}{|c|c|c|c|}
\hline $\begin{array}{l}\text { Initiating } \\
\text { System }\end{array}$ & Description & Advantages & Disadvantages \\
\hline $\begin{array}{l}\text { Normal ATRP } \\
\text { (Braunecker } \\
\text { and } \\
\text { Matyjaszewski, } \\
\text { 2007) }\end{array}$ & $\begin{array}{l}\text {-Alkyl halide }(\mathrm{R}-\mathrm{X}) \\
\text { initiator, activator }\left(M t^{n} / L\right) \\
\text { and monomers are starting } \\
\text { materials. }\end{array}$ & $\begin{array}{l}\text { - Near instantaneous } \\
\text { initiation of all chains }\end{array}$ & $\begin{array}{l}\text { - Highly sensitive to oxidants. } \\
\text {-Requires special handling } \\
\text { procedures. } \\
\text {-Needs large amount of metal } \\
\text { complex. } \\
\text {-Extensive post } \\
\text { polymerization purification } \\
\text { may be necessary. } \\
\text {-Very difficult to carry out in } \\
\text { aqueous media. }\end{array}$ \\
\hline Reverse ATRP & $\begin{array}{l}\text {-Conventional free radical } \\
\text { initiator, deactivators }(\mathrm{X}- \\
\left.\mathrm{Mt}^{\mathrm{q}+1} / \mathrm{L}\right) \text {, and monomers are } \\
\text { starting reagents. } \\
\text {-Activators }(\mathrm{Mt} / \mathrm{Li}) \text { and } \\
\text { ATRP initiators (alkyl } \\
\text { halide, } \mathrm{R}-\mathrm{X}) \text { are generated } \\
\text { in situ. }\end{array}$ & $\begin{array}{l}\text {-Initial components of the } \\
\text { system are not sensitive to } \\
\text { oxidants as standard } \\
\text { ATRP. } \\
\text {-Convenient to handle. }\end{array}$ & $\begin{array}{l}\text {-Block copolymers cannot be } \\
\text { formed with this technique. } \\
\text {-Uses large amounts of metal } \\
\text { complex. }\end{array}$ \\
\hline SR and NI & $\begin{array}{l}\text {-Conventional free radical } \\
\text { initiators as well as ATRP } \\
\text { initiators, activators, } \\
\text { deactivators, and monomers } \\
\text { are used as starting } \\
\text { reagents. } \\
\text {-Radicals generated from } \\
\text { conventional free radical } \\
\text { initiators act as reducing } \\
\text { agent to generate more } \\
\text { activators in the system. }\end{array}$ & $\begin{array}{l}\text {-Initial components of the } \\
\text { system are not as sensitive } \\
\text { to oxidants as standard } \\
\text { ATRP } \\
\text {-Block copolymers can be } \\
\text { formed } \\
\text {-Can be successfully } \\
\text { employed in emulsion. }\end{array}$ & $\begin{array}{l}\text {-Cannot produce clean block } \\
\text { copolymers (homopolymers } \\
\text { are formed along with the } \\
\text { block copolymers). } \\
\text {-Uses large amounts of metal } \\
\text { complex as compared to } \\
\text { ARGET ATRP. }\end{array}$ \\
\hline AGET & $\begin{array}{l}\text {-Reducing agent, } \\
\text { deactivator, and monomers } \\
\text { are used as starting agents. } \\
\text {-Activators are generated in } \\
\text { situ }\end{array}$ & $\begin{array}{l}\text {-Clean block copolymers } \\
\text { are formed } \\
\text {-Particularly useful in } \\
\text { aqueous systems. }\end{array}$ & $\begin{array}{l}\text { - Compared to ARGET, } \\
\text { electrochemical and photo } \\
\text { induced ATRP, it uses a large } \\
\text { amounts of metal complex. } \\
\text {-Metal complex often needs to } \\
\text { be purified from product. }\end{array}$ \\
\hline $\begin{array}{l}\text { Hybrid and } \\
\text { bimetallic } \\
\text { catalytic } \\
\text { system }\end{array}$ & $\begin{array}{l}\text {-Uses immobilized } \\
\text { activators. } \\
\text {-Soluble efficient } \\
\text { deactivator, and monomers } \\
\text { are starting reagents. } \\
\text {-efficient deactivator } \\
\text { regenerates immobilized } \\
\text { activators due to their high } \\
\text { affinity to halogen atoms. }\end{array}$ & $\begin{array}{l}\text {-Allows for easy } \\
\text { purification of metal } \\
\text { catalyst by filtration. }\end{array}$ & $\begin{array}{l}\text {-Requires purification } \\
\text { processes. } \\
\text {-Requires that relatively high } \\
\text { amounts of metal complexes } \\
\text { be employed. } \\
\text {-Less control over MW and } \\
\text { MWD. }\end{array}$ \\
\hline
\end{tabular}




\begin{tabular}{|c|c|c|c|}
\hline ICAR & $\begin{array}{l}\text {-Conventional radical } \\
\text { initiators, activators, and } \\
\text { monomers are used as } \\
\text { starting agents. } \\
\text {-Differs from SR\&NI due } \\
\text { to slow decomposition of } \\
\text { initiator over } \\
\text { polymerization and the fact } \\
\text { that large amount of excess } \\
\text { free radicals used. } \\
\text {-Activators are regenerated } \\
\text { from deactivators due to } \\
\text { free radical initiator. }\end{array}$ & $\begin{array}{l}\text {-Metal complex } \\
\text { concentration in ppm } \\
\text {-Oxidants are scavenged } \\
\text { by generated activators } \\
\text { and excess initiators. }\end{array}$ & $\begin{array}{l}\text { - Homopolymers are formed } \\
\text { along with the block } \\
\text { copolymers. } \\
\text {-Small chain impurities are } \\
\text { formed }\end{array}$ \\
\hline ARGET & $\begin{array}{l}\text {-Reducing agent, ATRP } \\
\text { initiators (R-X), activators } \\
\text { and monomers are used as } \\
\text { starting reagents. } \\
\text {-Reducing agent used to } \\
\text { regenerate activators from } \\
\text { deactivators. }\end{array}$ & $\begin{array}{l}\text {-Metal complex } \\
\text { concentration in ppm. } \\
\text {-Oxidants are scavenged } \\
\text { by generated activators } \\
\text { and excess initiators. }\end{array}$ & $\begin{array}{l}\text { - Reducing agent often needs } \\
\text { to be purified from product. }\end{array}$ \\
\hline $\begin{array}{l}\text { eATRP } \\
\text { (Hosseiny and } \\
\text { van Rijn, 2013) }\end{array}$ & $\begin{array}{l}\text {-Monomers and activators } \\
\text { are used as starting } \\
\text { reagents. } \\
\text {-Electrochemical processes } \\
\text { used to regenerate } \\
\text { activators. }\end{array}$ & $\begin{array}{l}\text {-Cleanest method. } \\
\text { - Metal complex } \\
\text { concentration in ppm. } \\
\text {-Oxidants are scavenged. } \\
\text {-Polymerization can be } \\
\text { turned on/off. }\end{array}$ & -Requires complicated reactor. \\
\hline $\begin{array}{l}\text { Photo induced } \\
\text { ATRP } \\
\text { (Chantasirichot } \\
\text { et al., 2015; } \\
\text { Dadashi-Silab } \\
\text { et al., 2014; } \\
\text { Konkolewicz } \\
\text { et al., 2012) }\end{array}$ & $\begin{array}{l}\text {-Monomers and activators } \\
\text { are used as starting } \\
\text { reagents. } \\
\text {-Light radiation used to } \\
\text { generate activators. }\end{array}$ & $\begin{array}{l}\text {-Small amount of metal } \\
\text { complex. } \\
\text {-Low temperature. } \\
\text {-Broad range of } \\
\text { monomers. }\end{array}$ & $\begin{array}{l}\text {-Not applicable for light } \\
\text { sensitive monomers. } \\
\text {-May need expensive metal } \\
\text { ligand complexes. }\end{array}$ \\
\hline $\begin{array}{l}\text { Metal free } \\
\text { ATRP (Treat et } \\
\text { al., 2014) }\end{array}$ & $\begin{array}{l}\text {-Monomers and organic } \\
\text { photo redox catalyst are } \\
\text { used as starting reagents. } \\
\text {-Light radiation activates } \\
\text { the catalyst. }\end{array}$ & $\begin{array}{l}\text { - Complete avoidance of } \\
\text { transition metals. } \\
\text {-Friendly for Bio and } \\
\text { microelectronic } \\
\text { applications. }\end{array}$ & $\begin{array}{l}\text {-Developing field and lack of } \\
\text { sufficient literature. } \\
\text {-Noncommercial Reagents } \\
\text { (photo redox catalyst). }\end{array}$ \\
\hline
\end{tabular}




\section{Appendix B: Polymerization in aqueous dispersed media}

Suspension Polymerization: It starts with the mixture of monomer, water, surfactant /emulsifier and monomer soluble initiator. Polymerization occurs inside monomer droplet stabilized by surfactant. Stirring is normally required throughout the whole process to prevent aggregation of particles. It contains particles with broad PSD (particle size distribution), usually between 10-500 $\mu \mathrm{m}$ (Arshady, 1992).

Dispersion Polymerization: It is a special case of precipitation polymerization where a polymeric stabilizer is added in the reaction medium. In this polymerization, the selected medium must be a good solvent for the monomer and stabilizer, but it must be a non-solvent for the polymer to be produced. The polymer particle produced in the homogeneous solution gets coated with stabilizer. Nucleation stage determines the particle size distribution (PSD), usually short nucleation stage is necessary for uniform particles. Ideally no more particles or coagulum should be formed during the particle growth stage. Polymer particle size usually vary between 1-10 $\mu \mathrm{m}$ (Arshady, 1992).

Emulsion Polymerization: It is the most common industrial technique to produce polymer latex whose applications are found in coatings, adhesives, paints and other areas. It also starts with monomer (usually hydrophobic or sparingly soluble in water), surfactant, water and initiator (generally water soluble). Surfactant concentration must be above the critical micelle concentration (CMC), but not high enough to enclose all of monomer into the micelle. Hence, most of the monomer is aggregated as droplets (several $\mu \mathrm{m}$ in size) but are prevented from coalescence by surfactants on the surface. Partially, the monomer is found in monomer swollen micelles and tiny fraction is found dissolved in the aqueous phase (Li, 2012; Min, 2008; Min and Matyjaszewski, 2009).

Emulsion polymerization is supposed to proceed through three stages:

Interval I, also called particle nucleation period, gives birth of polymer particles either through micellar or through homogeneous nucleation. During this interval less than $10 \%$ monomer conversion occurs. It is characterized by the presence of all: monomer droplets, precursor particles and micelles. The disappearance of micelles ends interval I.

Interval II, contains monomer droplets and precursor particles only. Even though the volume fraction of monomer droplet is high, the number fraction of precursor particles is even higher, 
polymerization mainly occurs inside the polymeric particles. Monomer droplets function as monomer reservoirs. During interval II, the number of polymer particles remains constant and monomer diffuses from the droplets to the particles. Usually 10-40 \% monomer conversion occurs during interval II. End of interval II is marked by the disappearance of monomer droplets.

Interval III, is marked by the absence of monomer droplets and micelles. During this interval monomers remaining inside the particles continue to polymerize. Monomer conversion gets higher and there is significant increase in the medium viscosity. When all the monomer inside the particles is consumed or remain unusable, it marks the end of interval III, and hence the end of polymerization. Finally, polymer particles $(50-500 \mathrm{~nm})$ with relatively narrow size distribution are formed.

The average molecular weight of polymers obtained in emulsion polymerization (thorough FRP) are usually higher than those obtained in bulk/solution polymerizations.

Miniemulsion Polymerization: This polymerization is particularly much suitable for very hydrophobic monomers where monomer diffusion from droplets to polymerizing particles is relatively difficult. It differs from emulsion polymerization in the particle nucleation mechanism. Very high shear force, such as ultra-sonication is used to generate nanometer $(\mathrm{nm})$ size monomer droplets that are prevented from coalescence/Ostwald ripening as small amount of surfactant/stabilizer is added into the system. Droplet nucleation is a predominant method of particle nucleation, whereas homogeneous and micellar nucleation are negligible. Monomer droplets are kinetically stable but thermodynamically unstable. Relatively uniform size of monomer droplets results in the formation of uniform particle size (low PSD, particle size distribution) with normal range 50-500 $\mathrm{nm}$. Compatibility for very hydrophobic monomer, need of less surfactant (below CMC condition) and less sensitivity to the initiator amount added, agitation speed and polymerization temperature are some of the advantages of miniemulsion polymerization (W. Li, 2012; Min, 2008; Min \& Matyjaszewski, 2009).

Microemulsion Polymerization: It is a thermodynamically stable and optically transparent/translucent dispersion system which is prepared by the spontaneous dispersion of monomer (oil) in water in the presence of excess surfactant (far above CMC condition). The mechanisms of microemulsion differs significantly from that of miniemulsion. Micellar nucleation is the primary process of nucleation and growth. No intense shear is required. Particle size can be as low as around $10 \mathrm{~nm}$, depending on the amount of surfactant. Non 
nucleated micelles function as monomer reservoirs. High level of surfactant is the distinct disadvantage of microemulsion polymerization (Candau et al., 1985; W. Li, 2012; Min \& Matyjaszewski, 2009).

The essential features of microemulsion polymerization are:

1. Only two intervals of emulsion polymerization are present. Where interval II is missing.

2. The average number of radicals per particle during polymerization is between $0.1-0.3$.

3. Particle size and number increases with conversion.

4. Polymer chains are highly compressed. 


\section{Appendix C: Design Evaluation}

Table C.1: Degrees of freedom for evaluation

\begin{tabular}{|c|c|}
\hline Source & $\begin{array}{c}\text { Degree of } \\
\text { freedom }\end{array}$ \\
\hline Model & 15 \\
\hline Residuals & 5 \\
\hline Lack of Fit & 1 \\
\hline Pure Error & 4 \\
\hline Corr Total & 20 \\
\hline
\end{tabular}

Leverages for each experimental run and center point run are: 0.9851 and 0.0476 respectively. The average leverage of the whole design is 0.7619 .

The evaluation of design is based on the ANOVA result.

Table C.2: Design Matrix Evaluation for Factorial 2FI Model

\begin{tabular}{ccccccc|}
\hline & & & \multicolumn{4}{c|}{$\begin{array}{c}\text { Power at 5 \% alpha level to detect } \\
\text { signal/noise ratios of }\end{array}$} \\
Term & StdErr $^{1}$ & VIF & Ri-Squared & 0.5 Std. Dev. & 1 Std. Dev. & 2 Std. Dev. \\
A & 0.25 & 1 & 0 & $13.00 \%$ & $36.80 \%$ & $88.70 \%$ \\
B & 0.25 & 1 & 0 & $13.00 \%$ & $36.80 \%$ & $88.70 \%$ \\
C & 0.25 & 1 & 0 & $13.00 \%$ & $36.80 \%$ & $88.70 \%$ \\
D & 0.25 & 1 & 0 & $13.00 \%$ & $36.80 \%$ & $88.70 \%$ \\
E & 0.25 & 1 & 0 & $13.00 \%$ & $36.80 \%$ & $88.70 \%$ \\
AB & 0.25 & 1 & 0 & $13.00 \%$ & $36.80 \%$ & $88.70 \%$ \\
AC & 0.25 & 1 & 0 & $13.00 \%$ & $36.80 \%$ & $88.70 \%$ \\
AD & 0.25 & 1 & 0 & $13.00 \%$ & $36.80 \%$ & $88.70 \%$ \\
AE & 0.25 & 1 & 0 & $13.00 \%$ & $36.80 \%$ & $88.70 \%$ \\
BC & 0.25 & 1 & 0 & $13.00 \%$ & $36.80 \%$ & $88.70 \%$ \\
BD & 0.25 & 1 & 0 & $13.00 \%$ & $36.80 \%$ & $88.70 \%$ \\
BE & 0.25 & 1 & 0 & $13.00 \%$ & $36.80 \%$ & $88.70 \%$ \\
CD & 0.25 & 1 & 0 & $13.00 \%$ & $36.80 \%$ & $88.70 \%$ \\
CE & 0.25 & 1 & 0 & $13.00 \%$ & $36.80 \%$ & $88.70 \%$ \\
DE & 0.25 & 1 & 0 & $13.00 \%$ & $36.80 \%$ & $88.70 \%$ \\
\hline
\end{tabular}

${ }^{1}$ Basis Std. Dev. $=1.0$

Standard errors should be similar within type of coefficient. Smaller is better. Ideal VIF is 1.0. VIFs above 10 are cause for alarm, indicating coefficients are poorly estimated due to multicollinearity. Ideal Ri-squared is 0.0 . High Ri-squared means terms are correlated with 
each other, possibly leading to poor models. Power should be approximately $80 \%$ for the effect desired to be detected.

Table C.3: Measures derived from the information matrix

\begin{tabular}{|ccc|}
\hline Run & Leverage & Space Type \\
1 & 0.9851 & Factorial \\
2 & 0.9851 & Factorial \\
3 & 0.9851 & Factorial \\
4 & 0.9851 & Factorial \\
5 & 0.0476 & Center \\
6 & 0.9851 & Factorial \\
7 & 0.9851 & Factorial \\
8 & 0.9851 & Factorial \\
9 & 0.9851 & Factorial \\
10 & 0.9851 & Factorial \\
11 & 0.9851 & Factorial \\
12 & 0.9851 & Factorial \\
13 & 0.9851 & Factorial \\
14 & 0.9851 & Factorial \\
15 & 0.0476 & Center \\
16 & 0.9851 & Factorial \\
17 & 0.9851 & Factorial \\
18 & 0.0476 & Center \\
19 & 0.0476 & Center \\
20 & 0.0476 & Center \\
21 & 0.9851 & Factorial \\
Average $=0.7619$ & \\
\hline & & \\
\hline
\end{tabular}

Leverage is the potential for a design point to influence the fit of the model coefficients, based on its position in the design space. A leverage of 1 means the model must exactly fit the observed value. A design for the same model but having more runs will tend to have lower leverage for each point. 


\section{Appendix D: Statistical Analysis}

Table D.1: Analysis of variance table [Partial sum of squares - Type III] for conversion

\begin{tabular}{|c|c|c|c|c|cc|}
\hline Source & $\begin{array}{c}\text { Sum of } \\
\text { Squares }\end{array}$ & df & $\begin{array}{c}\text { Mean } \\
\text { Square }\end{array}$ & $\begin{array}{c}\mathbf{F} \\
\text { Value }\end{array}$ & $\begin{array}{c}\boldsymbol{p} \text {-value } \\
\text { Prob }>\mathbf{F}\end{array}$ & \\
\hline Model & 1602.22 & 12 & 133.52 & 75.28 & $<0.0001$ & significant \\
A-Temperature & 1152.6 & 1 & 1152.6 & 649.9 & $<0.0001$ & \\
B-Catalyst Complex & 77.44 & 1 & 77.44 & 43.67 & 0.0003 & \\
C-Brij 98 & 152.52 & 1 & 152.52 & 86 & $<0.0001$ & \\
D-EBiB & 0.56 & 1 & 0.56 & 0.32 & 0.5909 & \\
E-Ascorbic acid & 43.56 & 1 & 43.56 & 24.56 & 0.0016 & \\
AB & 21.16 & 1 & 21.16 & 11.93 & 0.0106 & \\
AC & 68.06 & 1 & 68.06 & 38.38 & 0.0004 & \\
BC & 33.64 & 1 & 33.64 & 18.97 & 0.0033 & \\
BE & 15.6 & 1 & 15.6 & 8.8 & 0.0209 & \\
CD & 23.52 & 1 & 23.52 & 13.26 & 0.0083 & \\
CE & 6.25 & 1 & 6.25 & 3.52 & 0.1026 & \\
DE & 7.29 & 1 & 7.29 & 4.11 & 0.0822 & \\
Curvature & 1.06 & 1 & 1.06 & 0.6 & 0.4648 & \\
Residual & 12.41 & 7 & 1.77 & & & \\
Lack of Fit & 3.48 & 3 & 1.16 & 0.52 & 0.6911 & not significant \\
Pure Error & 8.93 & 4 & 2.23 & & & \\
Cor Total & 1615.69 & 20 & & & & \\
\hline
\end{tabular}

Table D.2: Coefficient estimate for conversion

\begin{tabular}{|c|c|c|c|c|c|c|}
\hline Factor & $\begin{array}{c}\text { Coefficient } \\
\text { Estimate }\end{array}$ & df & $\begin{array}{c}\text { Standard } \\
\text { Error }\end{array}$ & $\begin{array}{c}\mathbf{9 5 \%} \text { CI } \\
\text { Low }\end{array}$ & $\begin{array}{c}\mathbf{9 5 \%} \text { CI } \\
\text { High }\end{array}$ & VIF \\
\hline Intercept & 29.66 & 1 & 0.28 & 29.01 & 30.31 & \\
A-Temperature & 8.49 & 1 & 0.32 & 7.74 & 9.24 & 1 \\
B-Catalyst Complex & -2.2 & 1 & 0.32 & -2.95 & -1.45 & 1 \\
C-Brij 98 & 3.09 & 1 & 0.32 & 2.34 & 3.84 & 1 \\
D-EBiB & -0.19 & 1 & 0.32 & -0.94 & 0.56 & 1 \\
E-Ascorbic acid & 1.65 & 1 & 0.32 & 0.9 & 2.4 & 1 \\
AB & -1.15 & 1 & 0.32 & -1.9 & -0.4 & 1 \\
AC & 2.06 & 1 & 0.32 & 1.31 & 2.81 & 1 \\
BC & -1.45 & 1 & 0.32 & -2.2 & -0.7 & 1 \\
BE & 0.99 & 1 & 0.32 & 0.24 & 1.74 & 1 \\
CD & 1.21 & 1 & 0.32 & 0.46 & 1.96 & 1 \\
CE & 0.63 & 1 & 0.32 & -0.12 & 1.37 & 1 \\
DE & -0.67 & 1 & 0.32 & -1.42 & 0.073 & 1 \\
\hline
\end{tabular}


Table D.3: Analysis of variance table [Partial sum of squares - Type III] for molecular weight

\begin{tabular}{|c|c|c|c|c|cc|}
\hline Source & $\begin{array}{c}\text { Sum of } \\
\text { Squares }\end{array}$ & df & $\begin{array}{c}\text { Mean } \\
\text { Square }\end{array}$ & $\begin{array}{c}\mathbf{F} \\
\text { Value }\end{array}$ & $\begin{array}{c}\boldsymbol{p} \text {-value } \\
\text { Prob > F }\end{array}$ & \\
\hline Model & $5.14 \mathrm{E}+08$ & 12 & $4.28 \mathrm{E}+07$ & 23.7 & 0.0002 & significant \\
A-Temperature & $1.77 \mathrm{E}+08$ & 1 & $1.77 \mathrm{E}+08$ & 97.68 & $<0.0001$ & \\
B-Catalyst Complex & $1.49 \mathrm{E}+07$ & 1 & $1.49 \mathrm{E}+07$ & 8.26 & 0.0239 & \\
C-Brij 98 & $3.67 \mathrm{E}+05$ & 1 & $3.67 \mathrm{E}+05$ & 0.2 & 0.6661 & \\
D-EBiB & $1.20 \mathrm{E}+08$ & 1 & $1.20 \mathrm{E}+08$ & 66.53 & $<0.0001$ & \\
E-Ascorbic acid & $7.51 \mathrm{E}+06$ & 1 & $7.51 \mathrm{E}+06$ & 4.15 & 0.081 & \\
AB & $2.44 \mathrm{E}+07$ & 1 & $2.44 \mathrm{E}+07$ & 13.49 & 0.0079 & \\
AC & $9.88 \mathrm{E}+06$ & 1 & $9.88 \mathrm{E}+06$ & 5.47 & 0.052 & \\
AD & $7.87 \mathrm{E}+07$ & 1 & $7.87 \mathrm{E}+07$ & 43.52 & 0.0003 & \\
AE & $5.30 \mathrm{E}+06$ & 1 & $5.30 \mathrm{E}+06$ & 2.93 & 0.1307 & \\
BE & $3.99 \mathrm{E}+07$ & 1 & $3.99 \mathrm{E}+07$ & 22.09 & 0.0022 & \\
CD & $3.01 \mathrm{E}+07$ & 1 & $3.01 \mathrm{E}+07$ & 16.65 & 0.0047 & \\
Curvature & $1.82 \mathrm{E}+07$ & 1 & $1.82 \mathrm{E}+07$ & 10.04 & 0.0157 & \\
Residual & $1.27 \mathrm{E}+07$ & 7 & $1.81 \mathrm{E}+06$ & & & \\
Lack of Fit & $4.48 \mathrm{E}+06$ & 3 & $1.49 \mathrm{E}+06$ & 0.73 & 0.5856 & not significant \\
Pure Error & $8.18 \mathrm{E}+06$ & 4 & $2.04 \mathrm{E}+06$ & & & \\
Cor Total & $5.45 \mathrm{E}+08$ & 20 & & & & \\
\hline
\end{tabular}

Table D.4: Coefficient estimate for molecular weight

\begin{tabular}{|c|c|c|c|c|c|c|}
\hline Factor & $\begin{array}{c}\text { Coefficient } \\
\text { Estimate }\end{array}$ & df & $\begin{array}{c}\text { Standard } \\
\text { Error }\end{array}$ & $\begin{array}{c}\text { 95\% CI } \\
\text { Low }\end{array}$ & $\begin{array}{c}\text { 95\% CI } \\
\text { High }\end{array}$ & VIF \\
\hline Intercept & 14242.57 & 1 & 428.187145 & 13255.2 & 15230 & \\
A-Temperature & 3322 & 1 & 490.550001 & 2190.79 & 4453.21 & 1 \\
B-Catalyst Complex & -966 & 1 & 490.550001 & -2097.21 & 165.21 & 1 \\
C-Brij 98 & 151.375 & 1 & 490.550001 & -979.835 & 1282.59 & 1 \\
D-EBiB & -2741.63 & 1 & 490.550001 & -3872.84 & -1610.41 & 1 \\
E-Ascorbic acid & -685 & 1 & 490.550001 & -1816.21 & 446.21 & 1 \\
AB & -1234.75 & 1 & 490.550001 & -2365.96 & -103.54 & 1 \\
AC & -785.875 & 1 & 490.550001 & -1917.09 & 345.335 & 1 \\
AD & -2217.38 & 1 & 490.550001 & -3348.59 & -1086.16 & 1 \\
AE & -575.25 & 1 & 490.550001 & -1706.46 & 555.96 & 1 \\
BE & 1579.75 & 1 & 490.550001 & 448.54 & 2710.96 & 1 \\
CD & 1371.5 & 1 & 490.550001 & 240.29 & 2502.71 & 1 \\
\hline
\end{tabular}


Table D.5: Analysis of variance table [Partial sum of squares - Type III] for PDI

\begin{tabular}{|c|c|c|c|c|c|c|}
\hline Source & $\begin{array}{c}\text { Sum of } \\
\text { Squares }\end{array}$ & df & $\begin{array}{c}\text { Mean } \\
\text { Square }\end{array}$ & $\begin{array}{c}\mathbf{F} \\
\text { Value }\end{array}$ & $\begin{array}{c}p \text {-value } \\
\text { Prob }>\text { F }\end{array}$ & \\
\hline Model & 1.82 & 13 & 0.14 & 121.37 & $<0.0001$ & significant \\
\hline A-Temperature & 0.52 & 1 & 0.52 & 450.61 & $<0.0001$ & \\
\hline B-Catalyst Complex & 0.05 & 1 & 0.05 & 42.97 & 0.0006 & \\
\hline C-Brij 98 & 0.14 & 1 & 0.14 & 125.01 & $<0.0001$ & \\
\hline D-EBiB & $2.45 \mathrm{E}-03$ & 1 & $2.45 \mathrm{E}-03$ & 2.13 & 0.195 & \\
\hline E-Ascorbic acid & 0.24 & 1 & 0.24 & 210.97 & $<0.0001$ & \\
\hline $\mathrm{AC}$ & 0.11 & 1 & 0.11 & 95.68 & $<0.0001$ & \\
\hline $\mathrm{AE}$ & 0.14 & 1 & 0.14 & 117.23 & $<0.0001$ & \\
\hline $\mathrm{BC}$ & 0.016 & 1 & 0.016 & 14.22 & 0.0093 & \\
\hline $\mathrm{BD}$ & 0.088 & 1 & 0.088 & 76.57 & 0.0001 & \\
\hline $\mathrm{BE}$ & 0.17 & 1 & 0.17 & 151.3 & $<0.0001$ & \\
\hline $\mathrm{CD}$ & 0.082 & 1 & 0.082 & 71.5 & 0.0001 & \\
\hline $\mathrm{CE}$ & 0.24 & 1 & 0.24 & 205.45 & $<0.0001$ & \\
\hline $\mathrm{DE}$ & 0.016 & 1 & 0.016 & 14.11 & 0.0094 & \\
\hline Curvature & 0.045 & 1 & 0.045 & 39.22 & 0.0008 & \\
\hline Residual & $6.91 \mathrm{E}-03$ & 6 & $1.15 \mathrm{E}-03$ & & & \\
\hline Lack of Fit & $4.99 \mathrm{E}-03$ & 2 & $2.50 \mathrm{E}-03$ & 5.2 & 0.0771 & not significant \\
\hline Pure Error & $1.92 \mathrm{E}-03$ & 4 & $4.80 \mathrm{E}-04$ & & & \\
\hline Cor Total & 1.87 & 20 & & & & \\
\hline
\end{tabular}

Table D.6: Coefficient estimate for PDI

\begin{tabular}{|c|c|c|c|c|c|c|}
\hline Factor & $\begin{array}{c}\text { Coefficient } \\
\text { Estimate }\end{array}$ & df & $\begin{array}{c}\text { Standard } \\
\text { Error }\end{array}$ & $\begin{array}{c}\mathbf{9 5 \%} \text { CI } \\
\text { Low }\end{array}$ & $\begin{array}{c}\mathbf{9 5 \%} \text { CI } \\
\text { High }\end{array}$ & VIF \\
\hline Intercept & 1.40557143 & 1 & 0.018824 & 1.361059 & 1.450084 & \\
A-Temperature & 0.180125 & 1 & 0.021566 & 0.12913 & 0.23112 & 1 \\
B-Catalyst Complex & -0.055625 & 1 & 0.021566 & -0.10662 & -0.00463 & 1 \\
C-Brij 98 & 0.094875 & 1 & 0.021566 & 0.04388 & 0.14587 & 1 \\
D-EBiB & 0.012375 & 1 & 0.021566 & -0.03862 & 0.06337 & 1 \\
E-Ascorbic Acid & 0.12325 & 1 & 0.021566 & 0.072255 & 0.174245 & 1 \\
AC & 0.083 & 1 & 0.021566 & 0.032005 & 0.133995 & 1 \\
AE & 0.091875 & 1 & 0.021566 & 0.04088 & 0.14287 & 1 \\
BC & -0.032 & 1 & 0.021566 & -0.083 & 0.018995 & 1 \\
BD & 0.07425 & 1 & 0.021566 & 0.023255 & 0.125245 & 1 \\
BE & -0.104375 & 1 & 0.021566 & -0.15537 & -0.05338 & 1 \\
CD & -0.07175 & 1 & 0.021566 & -0.12275 & -0.02075 & 1 \\
CE & 0.121625 & 1 & 0.021566 & 0.07063 & 0.17262 & 1 \\
\hline
\end{tabular}




\section{REFERENCES}

Alam, M., Alam, A., Kamruzzaman, M., Kurihara, S., \& Saha, M. (2008). Development of a mathematical model by means of experimental design for alkylation of m-cresol with cyclopentene. Chemical Engineering Journal, 137(3), 598-602.

Arshady, R. (1992). Suspension, emulsion, and dispersion polymerization: a methodological survey. Colloid and Polymer Science, 270(8), 717-732.

Ayres, N. (2011). Atom Transfer Radical Polymerization: A Robust and Versatile Route for Polymer Synthesis. Polymer Reviews, 51(2), 138-162.

Barner-Kowollik, C., Vana, P., \& Davis, T. P. (2002). The Kinetics of Free-Radical Polymerization. In K. J. C. W. of N. Sciences \& T. P. Davis (Eds.), Handbook of Radical Polymerization (pp. 187-261).

Bebe, S. (2008). Estimation of free radical polymerization rate coefficients using computational chemistry.

Bergenudd, H. (2011). Understanding the mechanisms behind atom transfer radical polymerization exploring the limit of control. Stockholm:

Bertin, D., Gigmes, D., Le Mercier, C., Marque, S. R. A., \& Tordo, P. (2004). Factors Influencing $\mathrm{C}-\mathrm{ON}$ Bond Homolysis in Alkoxyamines: Unexpected Behavior of SG1 ( $N$-(2-methyl-2-propyl)- $N$-(1-diethylphosphono-2,2-dimethylpropyl)- $N$-oxyl)-Based Alkoxyamines. The Journal of Organic Chemistry, 69(15), 4925-4930.

Braunecker, W. A., \& Matyjaszewski, K. (2007). Controlled/living radical polymerization: Features, developments, and perspectives. Progress in Polymer Science, 32(1), 93-146.

Candau, F., Leong, Y. S., \& Fitch, R. M. (1985). Kinetic study of the polymerization of acrylamide in inverse microemulsion. Journal of Polymer Science: Polymer Chemistry Edition, 23(1), 193-214.

Chan, N., Cunningham, M. F., \& Hutchinson, R. A. (2013). Copper-mediated controlled radical polymerization in continuous flow processes: Synergy between polymer reaction engineering and innovative chemistry. Journal of Polymer Science Part A: Polymer Chemistry, 51(15), 3081-3096.

Chantasirichot, S., Inoue, Y., \& Ishihara, K. (2015). Photoinduced atom transfer radical polymerization in a polar solvent to synthesize a water-soluble poly(2- 
methacryloyloxyethyl phosphorylcholine) and its block-type copolymers. Polymer, 61, $55-60$.

Chauvin, F., Dufils, P.-E., Gigmes, D., Guillaneuf, Y., Marque, S. R. A., Tordo, P., \& Bertin, D. (2006). Nitroxide-Mediated Polymerization: The Pivotal Role of the $k_{\mathrm{d}}$ Value of the Initiating Alkoxyamine and the Importance of the Experimental Conditions. Macromolecules, 39(16), 5238-5250.

Cheng, C., Fu, Q., Bai, X., Liu, S., Shen, L., Fan, W., \& Li, H. (2013). Facile synthesis of gemini surface-active ATRP initiator and its use in soap-free AGET ATRP miniemulsion polymerisation. Chemical Papers, 67(3).

Cheng, C., Shu, J., Gong, S., Shen, L., Qiao, Y., \& Fu, C. (2010). Synthesis and use of a surface-active initiator in emulsion polymerization under AGET and ARGET ATRP conditions. New J. Chem., 34(1), 163-170.

Cheng, C.-J., Bai, X.-X., Fan, W.-Q., Wu, H.-M., Shen, L., Huang, Q.-H., \& Tu, Y.-M. (2014). Synthesis of a photoactive gemini surfactant and its use in AGET ATRP miniemulsion polymerisation and UV curing. Chemical Papers, 68(1).

Clark, A. J. (2002). Atom transfer radical cyclisation reactions mediated by copper complexes. Chemical Society Reviews, 31(1), 1-11.

Coessens, V. M. C., \& Matyjaszewski, K. (2010). Fundamentals of Atom Transfer Radical Polymerization. Journal of Chemical Education, 87(9), 916-919.

Coleman, B. D., \& Fox, T. G. (1963). A multistate mechanism for homogeneous ionic polymerization. II. The molecular weight distribution. Journal of the American Chemical Society, 85(9), 1241-1244.

Cunningham, M. F. (2003). Recent progress in nitroxide-mediated polymerizations in miniemulsion. Comptes Rendus Chimie, 6(11-12), 1351-1374.

Cunningham, M. F. (2008). Controlled/living radical polymerization in aqueous dispersed systems. Progress in Polymer Science, 33(4), 365-398.

Dadashi-Silab, S., Atilla Tasdelen, M., \& Yagci, Y. (2014). Photoinitiated atom transfer radical polymerization: Current status and future perspectives. Journal of Polymer Science Part A: Polymer Chemistry, 52(20), 2878-2888.

Destarac, M. (2010). Controlled Radical Polymerization: Industrial Stakes, Obstacles and Achievements. Macromolecular Reaction Engineering, 4(3-4), 165-179.

Drache, M., Schmidt-Naake, G., Buback, M., \& Vana, P. (2005). Modeling RAFT polymerization kinetics via Monte Carlo methods: cumyl dithiobenzoate mediated methyl acrylate polymerization. Polymer, 46(19), 8483-8493. 
Eslami, H., \& Zhu, S. (2005). Emulsion atom transfer radical polymerization of 2-ethylhexyl methacrylate. Polymer, 46(15), 5484-5493.

Eslami, H., \& Zhu, S. (2006). Emulsion atom transfer radical block copolymerization of 2ethylhexyl methacrylate and methyl methacrylate. Journal of Polymer Science Part A: Polymer Chemistry, 44(6), 1914-1925.

Features of Controlled "Living" Radical Polymerizations-Matyjaszewski Polymer Group Carnegie Mellon University.

Gaynor, S. G., Qiu, J., \& Matyjaszewski, K. (1998). Controlled/“Living” radical polymerization applied to water-borne systems. Macromolecules, 31(17), 5951-5954.

Goto, A., \& Fukuda, T. (2004). Kinetics of living radical polymerization. Progress in Polymer Science, 29(4), 329-385.

Greszta, D., Mardare, D., \& Matyjaszewski, K. (1994). “ Living” radical polymerization. 1. Possibilities and limitations. Macromolecules, 27(3), 638-644.

Guégain, E., Guillaneuf, Y., \& Nicolas, J. (2015). Nitroxide-Mediated Polymerization of Methacrylic Esters: Insights and Solutions to a Long-Standing Problem. Macromolecular Rapid Communications, 36(13), 1227-1247.

Guillaneuf, Y., Gigmes, D., Marque, S. R. A., Tordo, P., \& Bertin, D. (2006). NitroxideMediated Polymerization of Methyl Methacrylate Using an SG1-Based Alkoxyamine: How the Penultimate Effect Could Lead to Uncontrolled and Unliving Polymerization. Macromolecular Chemistry and Physics, 207(14), 1278-1288.

Guyot, A. (2002). Recent progress in reactive surfactants in emulsion polymerisation. Macromolecular Symposia, 179(1), 105-132.

He, G., \& Pan, Q. (2004). Synthesis of Polystyrene and Polystyrene/Poly(methyl methacrylate) Nanoparticles. Macromolecular Rapid Communications, 25(17), 1545-1548.

Hosseiny, S., \& van Rijn, P. (2013). Surface Initiated Polymerizations via e-ATRP in Pure Water. Polymers, 5(4), 1229-1240.

Kalil, S. J., Maugeri, F., \& Rodrigues, M. I. (2000). Response surface analysis and simulation as a tool for bioprocess design and optimization. Process Biochemistry, 35(6), 539550.

Kato, M., Kamigaito, M., Sawamoto, M., \& Higashimura, T. (1995). Polymerization of methyl methacrylate with the carbon tetrachloride/dichlorotris-(triphenylphosphine) ruthenium (II)/methylaluminum bis (2, 6-di-tert-butylphenoxide) initiating system: possibility of living radical polymerization. Macromolecules, 28(5), 1721-1723. 
Konkolewicz, D., Schröder, K., Buback, J., Bernhard, S., \& Matyjaszewski, K. (2012). Visible Light and Sunlight Photoinduced ATRP with ppm of Cu Catalyst. ACS Macro Letters, l(10), 1219-1223.

Lazić, Ž. R. (2004). Design and Analysis of Experiments: Section 2.3. In Design of Experiments in Chemical Engineering (pp. 262-367). Wiley-VCH Verlag GmbH \& Co. KGaA.

Li, H., Wang, W., Li, C., Tan, J., Yin, D., Zhang, H., Zhang, Q. (2015). Synthesis and characterization of brush-like multigraft copolymers PnBA-g-PMMA by a combination of emulsion AGET ATRP and emulsion polymerization. Journal of Colloid and Interface Science, 453, 226-236.

Li, W. (2012). Reactive Surfactants for Atom Transfer Radical Polymerization in Dispersed Media (Ph.D.). Carnegie Mellon University, United States -- Pennsylvania.

Li, W., \& Matyjaszewski, K. (2009). Star Polymers via Cross-Linking Amphiphilic Macroinitiators by AGET ATRP in Aqueous Media. Journal of the American Chemical Society, 131(30), 10378-10379.

Li, W., \& Matyjaszewski, K. (2011). Cationic Surface-Active Monomers as Reactive Surfactants for AGET Emulsion ATRP of $n$-Butyl Methacrylate. Macromolecules, 44(14), 5578-5585.

Louie, B. M., Carratt, G. M., \& Soong, D. S. (1985). Modeling the free radical solution and bulk polymerization of methyl methacrylate. Journal of Applied Polymer Science, 30(10), 3985-4012.

Mastan, E., Zhou, D., \& Zhu, S. (2014). Development of Molecular Weight Distribution in ATRP with Radical Termination: Development of MWD in ATRP. Macromolecular Theory and Simulations, 23(3), 227-240.

Matyjaszewski, K. (1996). Cationic Polymerizations: Mechanisms, Synthesis \& Applications. CRC Press.

Matyjaszewski, K. (1997). Mechanistic and synthetic aspects of atom transfer radical polymerization. Journal of Macromolecular Science, Part A: Pure and Applied Chemistry, 34(10), 1785-1801.

Matyjaszewski, K. (2002). General Concepts and History of Living Radical Polymerization. In

K. J. C. W. of N. Sciences \& T. P. Davis (Eds.), Handbook of Radical Polymerization (pp. 361-406). John Wiley \& Sons, Inc.

Matyjaszewski, K. (Ed.). (2003). Advances in Controlled/Living Radical Polymerization (Vol. 854). Washington, DC: American Chemical Society. 
Matyjaszewski, K. (2012). Atom Transfer Radical Polymerization: From Mechanisms to Applications. Israel Journal of Chemistry, 52(3-4), 206-220.

Matyjaszewski, K. (2014). From cationic ring-opening polymerization to atom transfer radical polymerization Polimery, 59(1), 24.

Matyjaszewski, K. The importance of exchange reactions in controlled/living radical polymerization in the presence of alkoxyamines and transition metals. Macromolecular Symposia, 111(1), 47-61.

Matyjaszewski, K., Paik, H., Zhou, P., \& Diamanti, S. J. (2001). Determination of Activation and Deactivation Rate Constants of Model Compounds in Atom Transfer Radical Polymerization. Macromolecules, 34(15), 5125-5131.

Matyjaszewski, K., Patten, T. E., \& Xia, J. (1997). Controlled/“living” radical polymerization. Kinetics of the homogeneous atom transfer radical polymerization of styrene. Journal of the American Chemical Society, 119(4), 674-680.

Matyjaszewski, K., Sumerlin, B. S., \& Tsarevsky, N. V. (2012). Progress in controlled radical polymerization: mechanisms and techniques. American Chemical Society.

Matyjaszewski, K., \& Xia, J. (2001). Atom Transfer Radical Polymerization. Chemical Reviews, 101(9), 2921-2990.

Min, K. (2008). Atom transfer radical polymerization in aqueous dispersed media (Ph.D.). Carnegie Mellon University, United States -- Pennsylvania.

Min, K., Gao, H., \& Matyjaszewski, K. (2006). Development of an ab Initio Emulsion Atom Transfer Radical Polymerization: From Microemulsion to Emulsion. Journal of the American Chemical Society, 128(32), 10521-10526.

Min, K., Gao, H., Yoon, J. A., Wu, W., Kowalewski, T., \& Matyjaszewski, K. (2009). OnePot Synthesis of Hairy Nanoparticles by Emulsion ATRP. Macromolecules, 42(5), 1597-1603.

Min, K., Jakubowski, W., \& Matyjaszewski, K. (2006). AGET ATRP in the Presence of Air in Miniemulsion and in Bulk. Macromolecular Rapid Communications, 27(8), 594-598.

Min, K., \& Matyjaszewski, K. (2005). Atom Transfer Radical Polymerization in Microemulsion. Macromolecules, 38(20), 8131-8134.

Min, K., \& Matyjaszewski, K. (2009). Atom transfer radical polymerization in aqueous dispersed media. Open Chemistry, 7(4).

Minisci, F. (1975). Free-radical additions to olefins in the presence of redox systems. Accounts of Chemical Research, 8(5), 165-171. 
Monteiro, M. J., \& Cunningham, M. F. (2012). Polymer Nanoparticles via Living Radical Polymerization in Aqueous Dispersions: Design and Applications. Macromolecules, 45(12), 4939-4957.

Montgomery, D. C. (2012). Design and Analysis of Experiments. Wiley.

Murari, A. R., Contant, S., Lona, L. M. F., Vivaldo-Lima, E., McManus, N. T., \& Penlidis, A. (2011). Modeling Insights on the TEMPO Mediated Radical Polymerization of Styrene. Journal of Macromolecular Science, Part A, 48(9), 681-687.

Nabifar, A. (2007). Investigations of kinetic aspects in nitroxide-mediated radical polymerization of styrene.

Natrella, M. (2010). NIST/SEMATECH e-Handbook of Statistical Methods. NIST/SEMATECH. Retrieved from http://www.itl.nist.gov/div898/handbook/

Odian, G. (2004). Principles of Polymerization. John Wiley \& Sons.

Oh, J. K. (2008). Recent advances in controlled/living radical polymerization in emulsion and dispersion. Journal of Polymer Science Part A: Polymer Chemistry, 46(21), 69837001 .

Okubo, M., Minami, H., \& Zhou, J. (2004). Preparation of block copolymer by atom transfer radical seeded emulsion polymerization. Colloid \& Polymer Science, 282(7), 747-752.

Pan, G., Sudol, E. D., Dimonie, V. L., \& El-Aasser, M. S. (2002). Surfactant Concentration Effects on Nitroxide-Mediated Living Free Radical Miniemulsion Polymerization of Styrene. Macromolecules, 35(18), 6915-6919.

Penlidis, A. (1986). Polymer reactor design, optimization and control in latex production technology.

Pintauer, T., \& Matyjaszewski, K. (2008). Atom transfer radical addition and polymerization reactions catalyzed by ppm amounts of copper complexes. Chemical Society Reviews, 37(6), 1087

Qu, J.-B., Huan, G.-S., Chen, Y.-L., Shao, H.-H., \& Wang, L. (2013). Exploration of the Bulk Atom Transfer Radical Polymerization of Styrene. Advances in Polymer Technology, 32(4).

Save, M., Guillaneuf, Y., \& Gilbert, R. G. (2006). Controlled Radical Polymerization in Aqueous Dispersed Media. Australian Journal of Chemistry, 59(10), 693-711.

Seeliger, F., \& Matyjaszewski, K. (2009). Temperature Effect on Activation Rate Constants in ATRP: New Mechanistic Insights into the Activation Process. Macromolecules, 42(16), 6050-6055. 
Shipp, D. A. (2005). Living Radical Polymerization: Controlling Molecular Size and Chemical Functionality in Vinyl Polymers. Journal of Macromolecular Science, Part C: Polymer Reviews, 45(2), 171-194.

Stoffelbach, F., Griffete, N., Bui, C., \& Charleux, B. (2008). Use of a simple surface-active initiator in controlled/living free-radical miniemulsion polymerization under AGET and ARGET ATRP conditions. Chemical Communications, (39), 4807.

Szwarc, M. (1956). “Living” polymers. Nature, 178(4543), 1168-1169.

Tang, W., Kwak, Y., Braunecker, W., Tsarevsky, N. V., Coote, M. L., \& Matyjaszewski, K. (2008). Understanding Atom Transfer Radical Polymerization: Effect of Ligand and Initiator Structures on the Equilibrium Constants. Journal of the American Chemical Society, 130(32), 10702-10713.

Teo, V. L., Davis, B. J., Tsarevsky, N. V., \& Zetterlund, P. B. (2014). Successful Miniemulsion ATRP Using an Anionic Surfactant: Minimization of Deactivator Loss by Addition of a Halide Salt. Macromolecules, 47(18), 6230-6237.

Toutenburg, H., \& Shalabh. (2009). Statistical Analysis of Designed Experiments, Third Edition. Springer Science \& Business Media.

Treat, N. J., Sprafke, H., Kramer, J. W., Clark, P. G., Barton, B. E., Read de Alaniz, J., ... Hawker, C. J. (2014). Metal-Free Atom Transfer Radical Polymerization. Journal of the American Chemical Society, 136(45), 16096-16101.

Ulitin, N. V., Nasyrov, I. I., Deberdeev, T. R., \& Berlin, A. A. (2012). Kinetic approach to modeling the radical polymerization of styrene in the presence dibenzyl trithiocarbonate. Russian Journal of Physical Chemistry B, 6(6), 752-760.

Ulrich, H. (1993). Introduction to Industrial Polymers. Hanser Publishers, 1993, 188.

Vana, P., Davis, T. P., \& Barner-Kowollik, C. (2002). Kinetic analysis of reversible addition fragmentation chain transfer (RAFT) polymerizations: conditions for inhibition, retardation, and optimum living polymerization. Macromolecular Theory and Simulations, 11(8), 823-835.

Wang, J.-S., \& Matyjaszewski, K. (1995). Controlled/“ living” radical polymerization. Atom transfer radical polymerization in the presence of transition-metal complexes. Journal of the American Chemical Society, 117(20), 5614-5615.

Wei, Y., Jia, Y., Wang, W.-J., Li, B.-G., \& Zhu, S. (2014). Surfactant-Ligand Design for $a b$ Initio Emulsion Atom Transfer Radical Polymerization. Macromolecules, 47(22), 7701-7706. 
Wei, Y., Liu, P., Wang, W.-J., Li, B.-G., \& Zhu, S. (2015). Well-controlled and stable emulsion ATRP of MMA with low surfactant concentration using surfactant-ligand design as the copper capture agent. Polym. Chem., 6(15), 2837-2843.

Wu, M., Forero Ramirez, L. M., Rodriguez Lozano, A., Quémener, D., Babin, J., Durand, A., ... Nouvel, C. (2015). First multi-reactive dextran-based inisurf for atom transfer radical polymerization in miniemulsion. Carbohydrate Polymers, 130, 141-148.

Xia, J., Gaynor, S. G., \& Matyjaszewski, K. (1998). Controlled/“living” radical polymerization. Atom transfer radical polymerization of acrylates at ambient temperature. Macromolecules, 31(17), 5958-5959.

Yang, B. (2014). Investigation of AGET-ATRP of methyl methacrylate in surface-active ionic liquid microemulsions. Iranian Polymer Journal, 23(2), 87-92.

Zetterlund, P. B., Kagawa, Y., \& Okubo, M. (2008). Controlled/Living Radical Polymerization in Dispersed Systems. Chemical Reviews, 108(9), 3747-3794.

Zhang, M., \& Ray, W. H. (2001). Modeling of "Living" Free-Radical Polymerization with RAFT Chemistry. Industrial \& Engineering Chemistry Research, 40(20), 4336-4352.

Zhou, Y., Qiu, L., Deng, Z., Texter, J., \& Yan, F. (2011). Low-Temperature AGET ATRP of Methyl Methacrylate in Ionic Liquid-Based Microemulsions. Macromolecules, 44(20), 7948-7955. 